\title{
Cooling Electrons in Nanoelectronic Devices by On-Chip Demagnetisation
}

\author{
Alexander Thomas Jones, MA (Hons) MSci (Hons) Camb
}

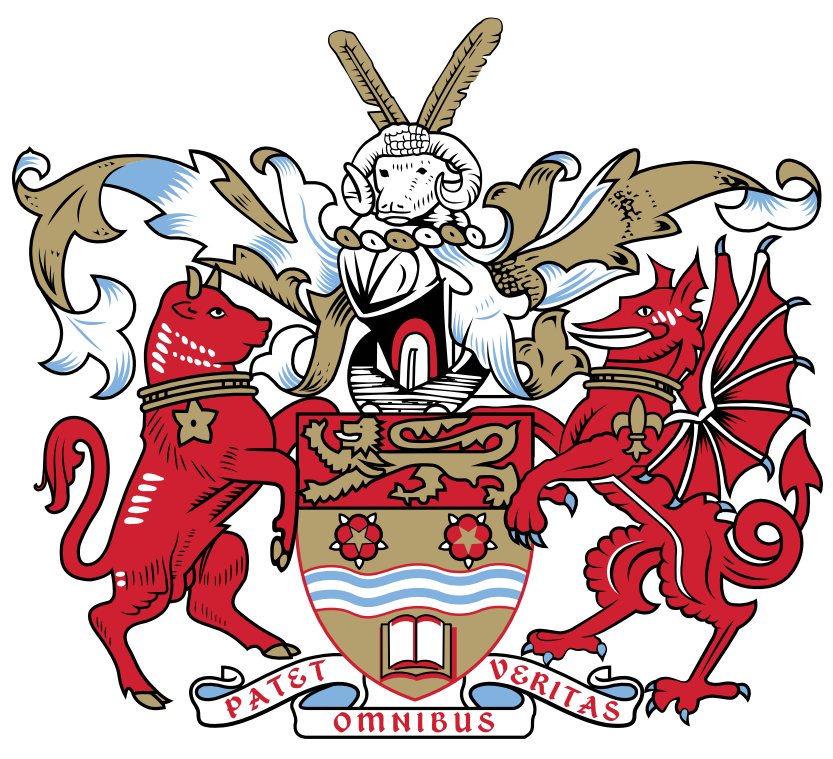

This thesis is submitted in partial fulfilment of the requirements for the degree of Doctor of Philosophy.

\section{\begin{tabular}{l|l} 
Physics & Lancaster \\
University
\end{tabular}}

Physics Department Lancaster University

September 2019 
(C) Alexander Thomas Jones: Cooling Electrons in Nanoelectronic Devices by OnChip Demagnetisation, PhD, Physics, Lancaster, September 2019 


\section{Declaration}

I declare that this thesis is my own work, and has not been submitted in substantially the same form for the award of a higher degree elsewhere.

I declare that this thesis is 29215 words long, and therefore confirm it does not exceed the permitted maximum of 80000 words.

Alexander Jones

September 2019 
BLANK 
Alexander Thomas Jones MA (Hons) MSci (Hons) Camb

Cooling Electrons in Nanoelectronic Devices by On-Chip Demagnetisation

Doctor of Philosophy

September 2019

\section{Abstract}

This thesis describes a novel cooling technique which allows the electrons within nanoelectronic devices to reach new low temperatures: nuclear demagnetisation of copper refrigerant mounted directly onto the chip a device is constructed on. This is within a field which has expanded in interest in recent years, due to the promise of new low electron temperatures allowing the investigation of new physical phenomena, the better fidelity of fundamental quantum effects and the improvement in quantum technologies such as quantum computers and sensors. Throughout the study, the effectiveness of the new technique is verified by applying it to a Coulomb blockade thermometer (CBT), a nanoelectronic device which provides primary (accurate without any need for calibration) thermometry of its own internal electron temperature. This thesis follows the development of this technique, starting from the initial proof of concept measurements made using a commercial, cryogen free, dilution refrigerator, as would be found in many low temperature and quantum transport laboratories. Here, the device electrons were cooled from $7 \mathrm{mK}$, the base temperature of the dilution refrigerator, to $4.5 \mathrm{mK}$ without using any other elaborate experimental constructions, opening the technique up to many other laboratories. This technique was then furthered by applying it to a newly adapted CBT which has the lowest operation temperature capability yet reported of $\approx 300 \mu \mathrm{K}$. This was done in a dilution refrigerator custom built in Lancaster, resulting in a minimum electron temperature of $(1.20 \pm 0.03) \mathrm{mK}$. This has opened the door to a new temperature regime in which to study new quantum effects, and going forward this technique will therefore be applied to other devices in order to enable these further investigations. 
BLANK 


\section{Acknowledgements}

Throughout the writing of this thesis, and the measurements that went into it, I am very grateful to have been supported by a fantastic team of people. Special thanks must, of course, go to my supervisors, Yuri Pashkin and Jon Prance, who have provided invaluable support, guidance and advice throughout, from the initial set-up of the experiment all the way to proof reading this thesis.

Credit for making the brilliant current sources used for all the measurements must go to Stephen Holt and his team in the Lancaster Physics Electronic Workshop, and credit for making the many precise bits and pieces that held everything together must go to Alan Stokes and Martin Ward in the Ultra-Low Temperature Workshop. Dilution refrigerators are complex beasts, and I must thank Jon Prance for help with running the dry dilution refrigerator, Ian Bradley and Roch Shanen for help with Fridge 6, and Dima Zmeev and Samuli Autti for help with Fridge 4.

The lab has been a brilliant place to work, mainly because of all the great people within it who were always willing to help at the drop of a hat. As well as those above, I thank Rich Haley for his brilliant way with words, shielding me from much of the bureaucracy, and for introducing me to the lab one summer 6 years ago. I thank Tony Guénault and for his helpful advice throughout the experiments. Thanks to Andy Guthrie, Josh Chawner and Theo Noble for always being available to be grabbed when I quickly needed assistance.

Outside the lab, thanks to my many customers of coffee club (some of whom I still don't know), for giving me an excuse to step back and do something simple from time to time. Last, but not least, thank you to my friends here, past and present and too many to list, who made my time so enjoyable with lunch time games, discussions about anything and everything, and trips to the pub, cinema and countryside. 
BLANK 


\section{Contributions}

All three Coulomb blockade thermometers (CBTs) used in this study were designed and fabricated by K. Grigoras, D. Gunnarsson, H. Heikkinen and M. Prunnila at VTT, the Technical Research Centre of Finland, and by J. Penttilä and L. Roschier at Aivon Oy. The original, unmodified version of the code which describes a full tunnelling model of a CBT is from the open source project pyCBT [1], developed by J. Penttilä and L. Roschier at Aivon Oy. 
BLANK 


\section{List of Acronyms}

AC alternating current 31, 32, 57, 61, 62

ALD atomic layer deposition 73

AWG american wire gauge 33, 55

CBT Coulomb blockade thermometer v, ix, 2, 3, 11, 13, 18, 19, 22, 25, 27-41, 44, $45,47-49,51-54,56-79,81,82,85,87-91,93-96$

DC direct current 23, 26, 28, 31-33, 40, 58-62, 64, 65, 67, 72, 77

ESD electrostatic discharge 29, 34

FWHM full width at half maximum 12, 18, 59

MXC mixing chamber 4, 21, 22, 29, 30, 34, 35, 37-40, 42, 47, 51, 52, 54, 55, 63, $64,66,67,75,78,87,88$

NIS normal metal-insulator-superconductor 22

PCB printed circuit board 30, 32, 34, 39, 52, 53

PID proportional-integral-differential 66

$\mathbf{R F}$ radio frequency 34

SEM scanning electron microscope 26

SET single-electron transistor 2, 12, 13, 24, 25, 57

SNR signal to noise ratio 2, 33, 62, 66 
xii

TEM transmission electron microscope 73

VWR vibrating wire resonator 53, 75

XRD X-ray diffraction 89, 90 


\section{Contents}

Declaration

Abstract $\quad$ v

Acknowledgements vii

$\begin{array}{ll}\text { Contributions } & \text { ix }\end{array}$

List of Acronyms xi

Contents

List of Figures $\quad$ XV

List of Tables $\quad$ xvii

1 Introduction 1

2 Background 3

2.1 Cooling Techniques ................... . . 3

2.1.1 Dilution Refrigeration .............. . . 3

2.1.2 Magnetic Cooling . . . . . . . . . . . . . . . 5

2.2 Coulomb Blockade Thermometry . . . . . . . . . . . . . . . . 11

2.2.1 Outline ........................... 11

2.2.2 Orthodox Theory of Single Electron Tunnelling . . . . . . . 13

2.2.3 Practical Measurements . . . . . . . . . . . . . . 18

2.3 On-Chip Refrigeration . . . . . . . . . . . . . . . . . . . . 19

2.3.1 Motivation and Principles . . . . . . . . . . . . . 19

2.3.2 Techniques........................ 22

3 On-Chip Demagnetisation Cooling on a Cryogen-Free Dilution Refrigerator

3.1 Coulomb Blockade Thermometer Device . . . . . . . . . . . . . 25 
3.2 Experimental Set-Up . . . . . . . . . . . . . . . . . . . . . 28

3.2 .1 Mounting and Heatsinking . . . . . . . . . . . . . . . 28

3.2 .2 Electrical . . . . . . . . . . . . . . . . . . . 31

3.3 CBT Characteristics . . . . . . . . . . . . . . 34

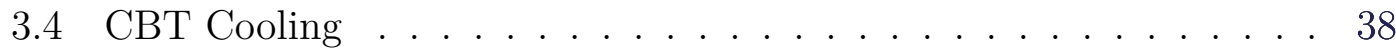

3.4 .1 Initial Experiments . . . . . . . . . . . . . . . . 38

3.4 .2 Thermal Modelling . . . . . . . . . . . . . . . . . . . 40

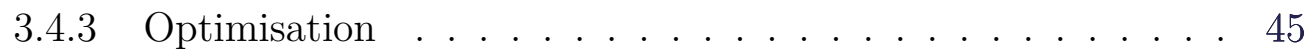

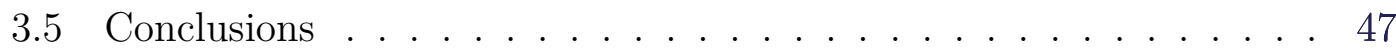

4 On-Chip Demagnetisation Cooling on a Cryogen-Filled Dilution Refrigerator 51

4.1 Experimental Set-Up . . . . . . . . . . . . . . . . . . . . 52

4.1 .1 Mounting and Heatsinking . . . . . . . . . . . . . . 52

4.1 .2 Electrical . . . . . . . . . . . . . . . 55

4.2 CBT Measurements . . . . . . . . . . . . . . . 56

4.2 .1 Additional Techniques . . . . . . . . . . . . . . 56

4.2 .2 Characterisation Results . . . . . . . . . . . . . . 63

4.3 Demagnetisation Cooling . . . . . . . . . . . . . . . . . 65

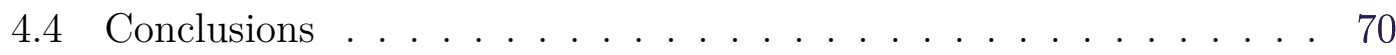

5 On-Chip Demagnetisation Cooling of a High Capacitance CBT 71

5.1 Experimental Set-Up . . . . . . . . . . . . . . . . . 72

5.1 .1 New CBT Fabrication . . . . . . . . . . . . . . 72

5.1 .2 Precooling . . . . . . . . . . . . . . . . . . . 74

5.2 CBT Characterisation . . . . . . . . . . . . 75

5.3 Demagnetisation Cooling . . . . . . . . . . . . . . . . 77

5.4 Heat Leaks . . . . . . . . . . . . . . . . . . . . . . 81

5.5 CBT Copper . . . . . . . . . . . . . . . . . 89

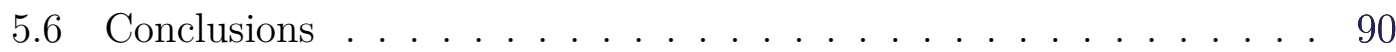

6 Summary and Outlook $\quad 93$

$\begin{array}{ll}\text { Bibliography } & 97\end{array}$ 


\section{List of Figures}

2.1 Schematic Dilution Refrigerator. . . . . . . . . . . . . 6

2.2 Entropy of Copper Nuclei in an Externally Applied Magnetic Field. 8

2.3 Coulomb Blockade on a Single Metallic Island. . . . . . . . . . . . . 13

2.4 Traditional Passive Cooling of a Nanoelectronic Device. . . . . . . . 20

2.5 Schematic energy diagram of an NIS cooler. . . . . . . . . . . . . 23

3.1 Coulomb Blockade Thermometer Chip. . . . . . . . . . . . . . . . 26

3.2 Coulomb Blockade Thermometer Junction Structure. . . . . . . . . 27

3.3 CBT Mounting in the Dry Cryostat. . . . . . . . . . . . . . . . 29

3.4 CBT Measurement Electrical Schematic. . . . . . . . . . . . . . . . 32

3.5 CBT Conductance Curve Broadening in Response to Noise. . . . . . 33

3.6 CBT Conductance Curves on a Dry Dilution Refrigerator at 0.1 T. . 35

3.7 CBT Conductance Curves on a Dry Dilution Refrigerator at 5.0 T. . 36

3.8 CBT Temperature vs. Conductance Self-Calibration Curve on a Dry Dilution Refrigerator. . . . . . . . . . . . . . . . 37

3.9 Thermalisation of the CBT on a Dry Dilution Refrigerator. . . . . . 38

3.10 CBT and MXC Warming During Magnetisation on a Dry Dilution Refrigerator. . . . . . . . . . . . . . 39

3.11 CBT Conductance During Demagnetisation at Different Biases on a Dry Dilution Refrigerator. . . . . . . . . . . . . . . 41

3.12 CBT Electron Temperature During Demagnetisation From 5.0 T at $2.5 \mathrm{mT} \mathrm{s}^{-1}$ on a Dry Dilution Refrigerator. . . . . . . . . . 42

3.13 Model of the Thermal Subsystems Within a CBT Island. . . . . . . 42

3.14 Thermal Model Fitted to a $2.5 \mathrm{mT} \mathrm{s}^{-1}$ Demagnetisation on a Dry Dilution Refrigerator. . . . . . . . . . . . . . . . 45

3.15 Evolution of $B / T_{e}$ Used for Demagnetisation Optimisation on a Dry Dilution Refrigerator. . . . . . . . . . . . . . . . 46

3.16 Effect of Demagnetisation Profile on CBT Temperature on a Dry Dilution Refrigerator. . . . . . . . . . . . . . . . . . 48

4.1 CBT Package Filter PCB. . . . . . . . . . . . . . . . . 53 
4.2 CBT Package Mounted on a Wet Dilution Refrigerator. . . . . . . . 54

4.3 Noise Isolation on the Wet Dilution Refrigerator. . . . . . . . . . 55

4.4 Locating the Conductance Minimum at MXC Temperature on a Wet Dilution Refrigerator. . . . . . . . . . . . . . . . . 60

4.5 Frequency Components of the Signal from a Simulated CBT. . . . . 62

4.6 CBT Conductance Curves on a Wet Dilution Refrigerator. . . . . . 63

4.7 Joule Heating During a CBT Conductance Curve Measurement on a Wet Dilution Refrigerator. . . . . . . . . . . . . . . . . . . . 64

4.8 CBT Temperature vs. Conductance Self-Calibration Curve on a Wet Dilution Refrigerator. . . . . . . . . . . . 65

4.9 Demagnetisation at $2.7 \mathrm{mT} \mathrm{s}^{-1}$ on a Wet Dilution Refrigerator. . . . 67

4.10 Thermal Model Fitted to a $2.7 \mathrm{mT} \mathrm{s}^{-1}$ Demagnetisation on a Wet Dilution Refrigerator. . . . . . . . . . . . . . 68

4.11 Effect of Demagnetisation Profile on CBT Temperature on a Wet Dilution Refrigerator. . . . . . . . . . . . . . . . . 69

5.1 Additional Stray Capacitance Added to a CBT. . . . . . . . . . . . 74

5.2 Cold Finger Used for the High Capacitance Measurements. . . . . . 76

5.3 High Capacitance CBT Conductance Curves on a Wet Dilution Refrigerator. . . . . . . . . . . . . . 76

5.4 High Capacitance CBT Self-Calibration Curve. . . . . . . . . . . . 78

5.5 High Capacitance CBT Electron Temperature During Demagnetisation from $8.0 \mathrm{~T}$ to $0.1 \mathrm{~T}$ at $3.0 \mathrm{mT} \mathrm{s}^{-1}$. . . . . . . . . . 79

5.6 Thermal Model Fitted to a $3.0 \mathrm{mT} \mathrm{s}^{-1}$ Demagnetisation of the High Capacitance CBT. . . . . . . . . . . . . . . . . . 79

5.7 Evolution of $B / T_{e}$ Used for Demagnetisation Optimisation with the High Capacitance CBT. . . . . . . . . . . . . . . . . . 80

5.8 Demagnetisation Profiles Summary. . . . . . . . . . . . . . . 82

5.9 Dynamic Heat Leaks with Sweep Rate. . . . . . . . . . . . . . . . . 84

5.10 Static Heat Leaks. . . . . . . . . . . . . . . . . . . . . . . 86

5.11 Fridge 4 Magnetic Heating Hysteresis. . . . . . . . . . . . . . . . . . 87

5.12 X-Ray Diffraction of the CBT Copper. . . . . . . . . . . . . . . . . 90 


\section{List of Tables}

3.1 CBT Sensor Parameters on a Dry Dilution Refrigerator. . . . . . . 37

4.1 CBT Sensor Parameters on a Wet Dilution Refrigerator. . . . . . . 64

5.1 CBT Heat Leaks at Constant Magnetic Field. . . . . . . . . . . . . 85 
xviii

BLANK 


\section{Chapter 1}

\section{Introduction}

Research into devices reliant on quantum effects, and into the quantum effects themselves, is often performed at very low temperatures. While some phenomena, such as the quantum Hall effect in graphene [2], can be observed at room temperature many others cannot and hence require some form of cooling. At its simplest, this cooling can take the form of an insulated container holding a boiling liquid cryogen; usually liquid nitrogen, sufficient for cooling to $77 \mathrm{~K}$ to allow observation of high temperature superconductivity in $\mathrm{YBCO}$ [3], or liquid helium providing cooling to $4.2 \mathrm{~K}$ at which an easily fabricated niobium superconducting quantum interference device (SQUID) [4] can operate.

Prior to this study, the state of the art for cooling quantum devices was the use of dilution refrigerators [5] which can achieve helium mixture temperatures as low as $1.75 \mathrm{mK}$ [6] for elaborate custom built machines, or more typically around $10 \mathrm{mK}$ for readily available commercial machines [7]. It is in these commercial dilution refrigerators that a significant part of today's cutting edge research into low temperature quantum technologies takes place. For example, superconducting qubits [810] for quantum computers, single electron turnstiles [11] for quantum metrology and superconducting quantum interference proximity transistors (SQUIPTs) [12] are all being actively explored at dilution refrigerator temperatures.

When working at temperatures significantly below $1 \mathrm{~K}$, the temperature of the electron bath in a nanoelectronic device diverges from its host lattice temperature due to the weakening of the electron-phonon coupling as temperature decreases [13]. The primary form of cooling for the electrons in most systems is via this coupling, to the lattice and then to some large low temperature reservoir (e.g. the mixing chamber flange of the dilution refrigerator). This weak electron-phonon coupling leads to elevated electron temperatures which can be of the order $100 \mathrm{mK}$ despite 
a mixing chamber temperature of $10 \mathrm{mK}$ or lower [14].

These elevated electron temperatures reduce the fidelity, or even lead to the absence of the desired quantum effect in the system being studied. For example quantum annealers, as used commercially by D-Wave Systems for quantum computation, benefit from the ability to accurately process larger scaled systems when operated at lower temperatures [15]. Also for some qubits, the charge noise [16] decreases and the coherence time increases [17] as the temperature decreases, bringing the goal of a practical quantum computer closer. Furthermore, lower electron temperatures help with ensuring that charge pumps [18] operate with a minimised leakage and back-tunnelling rate [19] and the signal to noise ratio (SNR) of single-electron transistor (SET) based sensors improves [20], furthering the field of quantum metrology.

This thesis describes work on the development and testing of on-chip magnetic cooling, a technique designed to realise these benefits. This is designed to cool the electrons in a nanoelectronic device directly without intervening phonons, thus bypassing the electron-phonon coupling thermal bottleneck. This is implemented by applying a thick $(\approx 6 \mu \mathrm{m})$ layer of electroplated copper on top of an array of aluminium islands making up a Coulomb blockade thermometer (CBT). This allows the temperature of interest, the electron temperature, to be easily and accurately read out during the cooling process.

The background theory and history of the cooling concepts used here, in addition to the history and theoretical descriptions of the CBT, are explained in Chapter 2 of this thesis. Chapter 2 also covers the other on-chip cooling work described in the literature prior to the commencement of this study. This is followed in Chapter 3 by our proof of concept work on the technique, where a CBT was cooled using nuclear demagnetisation refrigeration on a commercial, cryogen-free, dilution refrigerator. An initial attempt at replicating this technique using a dilution refrigerator custom built in Lancaster, in order to reach new low electron temperatures, is given in Chapter 4. This attempt cooled the CBT to the bottom of its operating range, where it was no longer able to accurately report the electron temperature. Hence, a second attempt was made using a different design of CBT, and the modifications that were necessary together with the results are presented in Chapter 5. Finally, this work is summarised, and future work discussed, in Chapter 6. 


\section{Chapter 2}

\section{Background}

This chapter describes the preliminary detail necessary for following the discussion concerning the new on-chip refrigeration technique discussed throughout the rest of this thesis. We start by considering a range of cooling techniques, that have traditionally been used for cooling samples below $1 \mathrm{~K}$, in Section 2.1. The theory, history and operation of CBTs is then given in Section 2.2. A summary of the other on-chip refrigeration studies, and an introduction to the technique used in this study, is then presented in Section 2.3.

\subsection{Cooling Techniques}

Like all other ultra-low temperature cooling techniques, the method described in this thesis is one step of a multi-stage process used to cool a sample from room to base temperature, the minimum temperature achievable for a given set of techniques. On-chip magnetic cooling is a final cooling stage, thus is pre-cooled by preceding stages. In this section, an overview of the prior stages is given in Subsection 2.1.1 which describes the dilution refrigerator in the context of the other necessary stages surrounding it. This is followed by Subsection 2.1.2 where nuclear demagnetisation refrigeration is described, this being the technique that is applied in a novel form to provide cooling of the on-chip electrons.

\subsubsection{Dilution Refrigeration}

For all iterations in the development of the new on-chip cooling technique, the device was pre-cooled using a dilution refrigerator. This provides a starting temperature that is easily reachable for laboratories experienced in low temperature techniques. The dilution refrigerator was proposed by H. London in 1951 and first 
realised in 1965 by P. Das et. al. [21] with a machine that reached a base temperature of $220 \mathrm{mK}$, significant because of it being lower than the lowest temperature achievable with the coldest continuous cooling technique of the day (the pumped ${ }^{3} \mathrm{He}$ cryostat which operates down to $\approx 300 \mathrm{mK}$ ). Today dilution refrigerators are well refined, and many labs have access to easy to use cryogen-free (dry) dilution refrigerators [22] or high performance dilution refrigerators with a liquid helium bath [6]. For all types the basic operation, as described below, is the same.

The dilution refrigerator is based upon the phenomenon that below $0.87 \mathrm{~K}$ a mixture of ${ }^{3} \mathrm{He}$ and ${ }^{4} \mathrm{He}$ spontaneously separates into two phases, one rich in ${ }^{3} \mathrm{He}$, the other dilute. In fact, as the temperature of the mixture approaches absolute zero, the rich phase tends towards pure ${ }^{3} \mathrm{He}$ while the dilute phase tends to a constant $6.6 \%{ }^{3} \mathrm{He}$ in ${ }^{4} \mathrm{He}[23]$.

This effect can be exploited by continuously circulating ${ }^{3} \mathrm{He}$; removing it from the dilute phase and then reintroducing it into the concentrated phase. This occurs in the mixing chamber (MXC) of the dilution refrigerator, which is a small volume located at the bottom of the cryostat with appropriate pipework to allow circulation to occur in this manner. This circulation would place the two phase system out of equilibrium due to the reduced concentration of ${ }^{3} \mathrm{He}$ in the dilute phase, so ${ }^{3} \mathrm{He}$ atoms move from the concentrated phase to the dilute. This 'diluting' of the ${ }^{3} \mathrm{He}$ has an associated enthalpy of mixing given by

$$
\Delta H \propto \int \Delta C d T
$$

for $\Delta C$ the difference in specific heats of the two phases as a function of temperature $T$. Both specific heats are proportional to $T$ at low temperatures, hence we achieve a cooling power

$$
\dot{Q} \propto \dot{n}_{3} T^{2}
$$

for a ${ }^{3}$ He circulation rate $\dot{n}_{3}$.

In a practical dilution refrigerator, shown schematically in Figure 2.1, the ${ }^{3} \mathrm{He}$ is removed from the dilute phase by carrying it to the 'still' under osmotic pressure where is is evaporated at around $700 \mathrm{mK}$. The ${ }^{3} \mathrm{He}$ is then re-condensed and reintroduced to the dilution refrigerator. This incoming liquid is further cooled using heat exchangers in contact with the outgoing ${ }^{3} \mathrm{He}$. In a 'wet' dilution refrigerator the condensation is performed using a pumped ${ }^{4} \mathrm{He}$ 'pot' operating at a nominal $1 \mathrm{~K}$, supplied and pre-cooled by a $4.2 \mathrm{~K}{ }^{4} \mathrm{He}$ bath surrounding the cryostat which can be thermally shielded by a surrounding bath of liquid nitrogen. In a 'dry' dilution refrigerator, the condensing is performed at a higher pressure to allow it 
to take place at the nominal $4 \mathrm{~K}$ stage of the mechanical cryocooler having been pre-cooled by the $50 \mathrm{~K}$ stage.

\subsubsection{Magnetic Cooling}

Magnetic cooling is a practical implementation of the magnetocaloric effect which was first discovered in iron [24]. Magnetic cooling operates by using an externally applied magnetic field to control magnetic disorder entropy of a paramagnetic substance. The paramagnetism is provided by a net dipole moment caused by the electrons or by the nuclear spin of the material. If each magnetic moment has total angular momentum quantum number $J$, then there are $2 J+1$ possible orientations (with respect to the orientation of the applied field) which are split in energy by the Zeeman effect. At high temperatures with low magnetic fields, the Zeeman splitting is much smaller than the thermal energy $k_{B} T$, so the orientations will be randomly distributed throughout the material leading to an entropy contribution of

$$
S=R \ln (2 J+1) .
$$

For $R$ the ideal gas constant. This is a general equation, applying to a material with an electronic magnetic moment or a nuclear magnetic moment, with $J$ representing either the total electron angular momentum or the nuclear spin. Demagnetisation of materials with an electronic magnetic moment, such as paramagnetic salts, is used to provide cooling to $2 \mathrm{mK}$, however the advent of dilution refrigerators makes this technique largely obsolete [23]. On the other hand, nuclear demagnetisation is still very relevant as it is the only cooling technique available for cooling bulk materials to below dilution refrigerator temperatures, and is the technique that we consider here. For adiabatic nuclear demagnetisation, the nuclear spin is conventionally denoted $I$, hence the entropy is often written as

$$
S=R \ln (2 I+1)
$$

At temperatures where magnetic cooling is feasible, this is the dominant entropy of the system. This permits the system's entropy to be controlled by varying the temperature of the system or the applied magnetic field. Below a certain temperature the thermal energy will become smaller than the Zeeman splitting, leading to energetically favourable orientations and hence spontaneous magnetic ordering. If a large external magnetic field is applied the Zeeman levels are further separated, hence magnetic ordering will occur at an elevated temperature [23].

To calculate the dependence of the entropy on total magnetic field $B$ and nuclear temperature $T_{n}$, we consider a nucleus with total spin quantum number $I$. The 


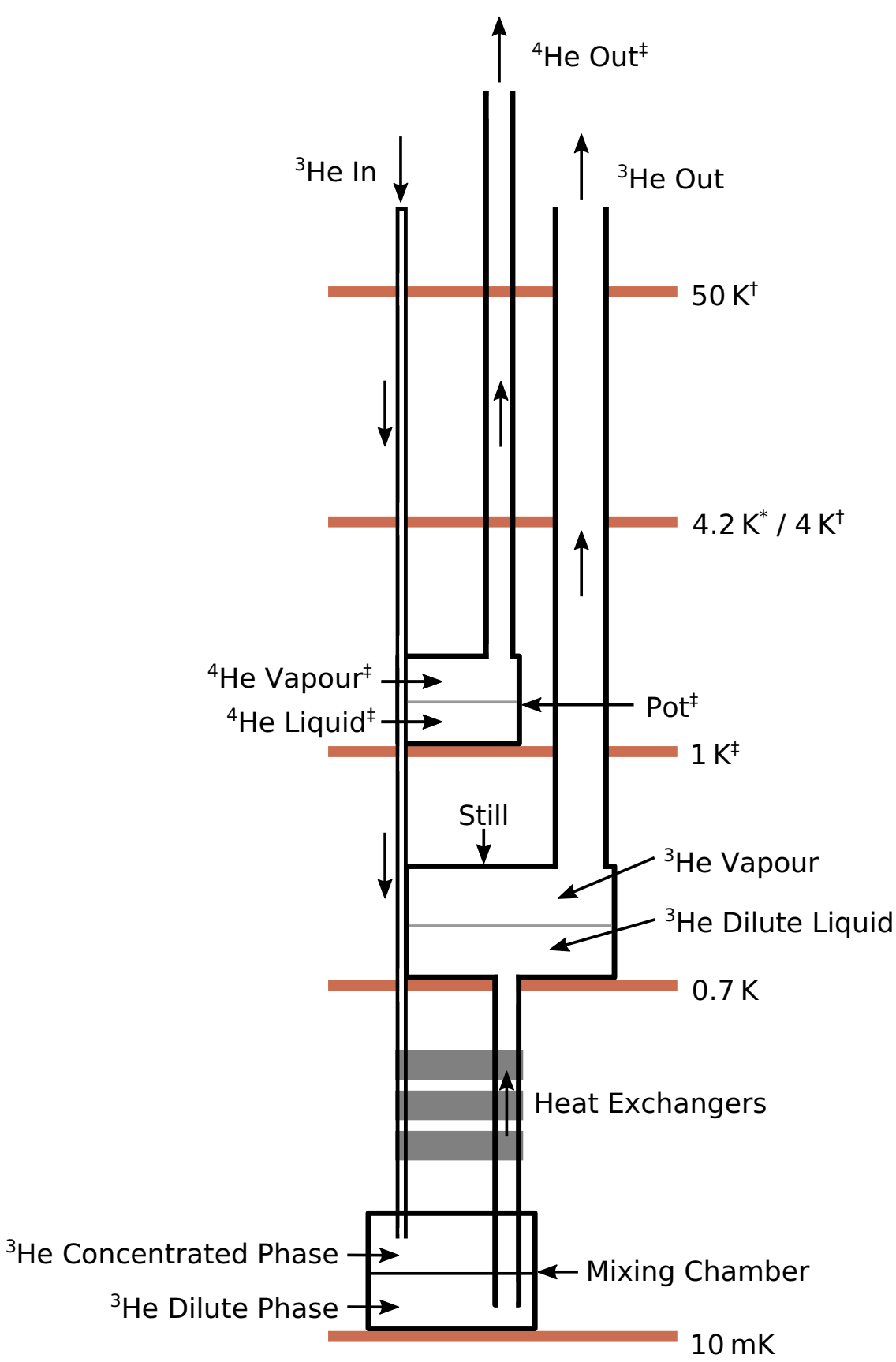

Figure 2.1: Schematic Dilution Refrigerator. The copper plates are labelled with their respective nominal temperatures. Items denoted $*$ and $\dagger$ are only present on wet and dry dilution refrigerators, respectively. Items denoted $\ddagger$ are usually omitted from dry dilution refrigerators. A condensing impedance for the incoming ${ }^{3} \mathrm{He}$ is heat sunk to the pot, or if a pot is not fitted, to the $4 \mathrm{~K}$ stage of the mechanical cryocooler. The incoming ${ }^{3} \mathrm{He}$ line is also heat sunk to the $4 \mathrm{~K} / 4.2 \mathrm{~K}$ stage and the still. 
Zeeman levels $\epsilon_{m},-I \leq m \leq I$, formed in a nucleus with an applied magnetic field are given by

$$
\epsilon_{m}=-m \mu_{N} g_{n} B
$$

where $\mu_{N}=e \hbar / 2 m_{p}$ is the nuclear magneton, for $\hbar$ the reduced Planck constant and $m_{p}$ the proton rest mass, and $g_{n}$ is the nuclear g-factor for the nuclei involved [25]. We can compute the partition function for a mole of some substance as

$$
Z=\left[\sum_{m=-I}^{I} \exp \left(-\beta \epsilon_{m}\right)\right]^{N_{A}}
$$

for $\beta=1 / k_{B} T_{n}$ and $N_{A}$ the Avogadro number. From this we can calculate the molar entropy using

$$
S=R \frac{\partial(T \ln Z)}{\partial T}
$$

which gives, after some manipulation,

$$
\begin{array}{r}
S=\frac{R}{2} x\left[\operatorname{coth}\left(\frac{1}{2} x\right)-(2 I+1) \operatorname{coth}\left(\frac{2 I+1}{2} x\right)\right] \\
+R \ln \left[\sinh \left(\frac{2 I+1}{2} x\right) / \sinh \left(\frac{1}{2} x\right)\right]
\end{array}
$$

for

$$
x=\frac{\mu_{N} g_{n}}{k_{B}} \frac{B}{T_{n}} .
$$

Figure 2.2 shows this function graphically for the example of copper, where the two naturally occurring and stable isotopes, ${ }^{63} \mathrm{Cu}$ and ${ }^{65} \mathrm{Cu}$, have spin $I=3 / 2$. From this, a thermodynamic cycle that can be used to provide cooling becomes apparent, as indicated in the figure. The prefactor of $x, \mu_{N} g_{n} / k_{B} \sim 5 \times 10^{-4} \mathrm{~K} \mathrm{~T}^{-1}$, is very small, reflecting the small size of the nuclear moments [23]. As such, $x \ll 1$ for most reasonable temperatures and magnetic fields used in nuclear demagnetisation. We can therefore expand Equation 2.8 to get

$$
S \approx R \ln (2 I+1)-\frac{\lambda_{n} B^{2}}{2 \mu_{0} T_{n}^{2}}
$$

for

$$
\lambda_{n}=N_{0} I(I+1) \mu_{0} \mu_{N}^{2} g_{n}^{2} / 3 k_{B}
$$

which is the molar nuclear Curie constant. Equation 2.10 shows that the entropy is entirely a function of $B / T_{n}$, which provides a useful figure of merit when planning and analysing demagnetisation cooling runs. We also note that at more modest magnetic fields, at dilution refrigerator temperatures, this reduces to Equation 2.4, as expected. During an ideal adiabatic demagnetisation, entropy is by definition 


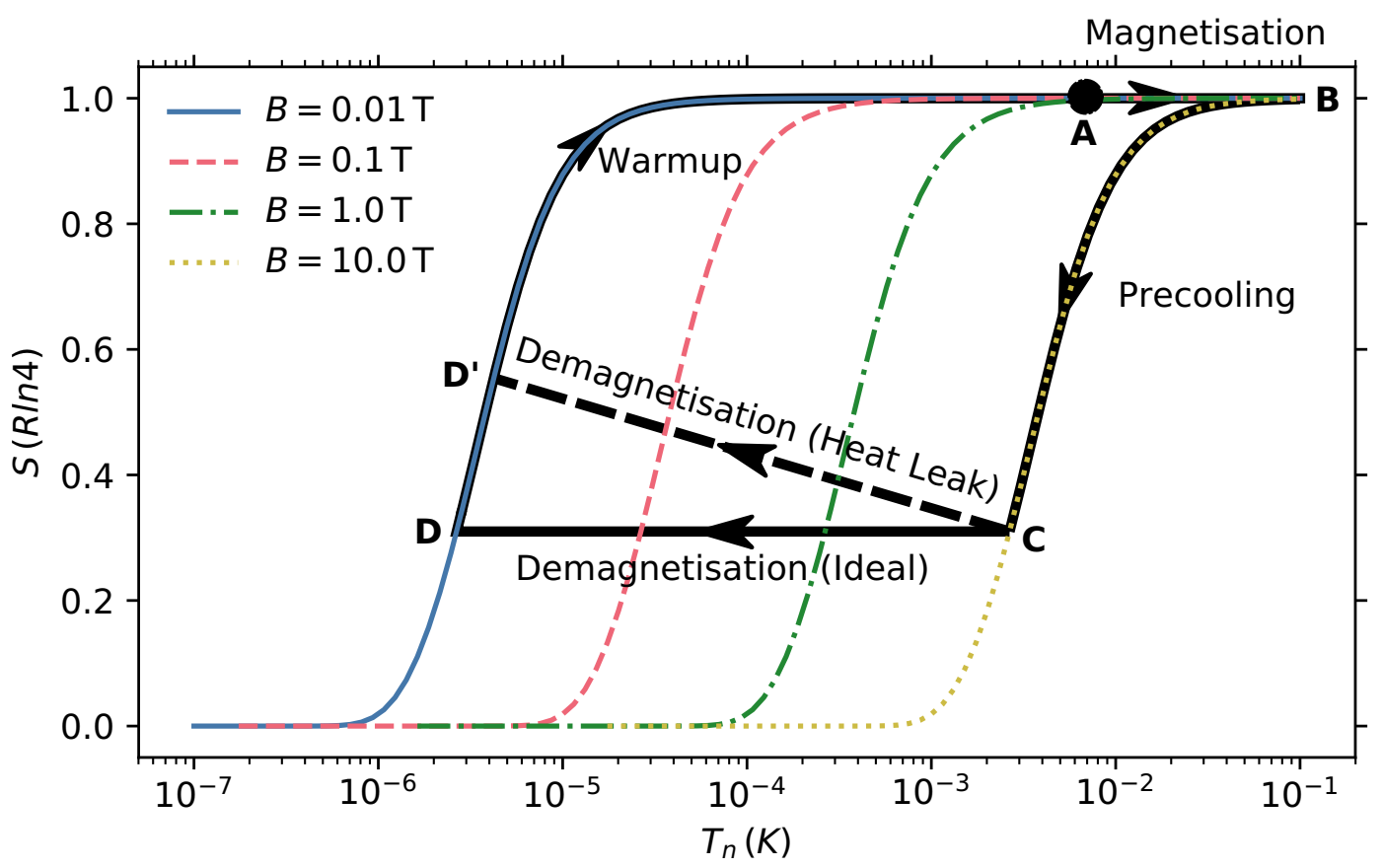

Figure 2.2: Entropy of Copper Nuclei in an Externally Applied Magnetic Field $\boldsymbol{B}$. The cycle used for adiabatic nuclear demagnetisation cooling [26] is shown with the solid black overlay: The nuclei are first magnetised by a large external magnetic field $(\mathbf{A}-\mathbf{B})$ and then allowed to cool towards the base temperature of the dilution refrigerator (precooling, $\mathbf{B}-\mathbf{C}$ ). Ideally the nuclei are then adiabatically demagnetised along the horizontal path $\mathbf{C}-\mathbf{D}$, however in reality some heat leak will be present leading to the following of the sloped dashed line to $\mathbf{C}-\mathbf{D}$ '. Finally, the nuclei warm up as the nuclear heat capacity is exhausted by the heat leak $(\mathbf{D}-\mathbf{A})$.

constant, hence so is the ratio $B / T_{n}$ allowing the calculation of the final temperature $T_{f}$ given the initial temperature $T_{i}$ and magnetic field $B_{i}$ together with the final magnetic field $B_{f}$ :

$$
T_{f}=T_{i} \frac{B_{f}}{B_{i}}
$$

It is important to note that $B$ is the total magnetic field sensed by the individual nuclear spins, not simply the externally applied field. The magnetic dipole interactions in the material's nuclei result in an internal magnetic field $b$ which becomes significant at low external fields. The two fields combine to give a total field of $B=\sqrt{B_{e x t}^{2}+b^{2}}$, limiting the minimum field and ultimate temperature that can be reached [23].

Nuclear demagnetisation cooling is usually performed using a large quantity of bulk nuclear refrigerant, usually $\mathrm{Cu}$ or $\mathrm{PrNi}_{5}$. The refrigerant is magnetised by applying a magnetic field of around $8 \mathrm{~T}$ using a superconducting solenoid. The resulting heat of magnetisation emitted by the refrigerant is removed to the ${ }^{3} \mathrm{He}^{-}{ }^{4} \mathrm{He}$ mixture in a dilution refrigerator [23] in a process termed 'precooling'. In order for this 
heat to flow towards the dilution refrigerator during precooling, but for no heat to flow back towards the cooled refrigerant during demagnetisation, a 'heat switch' is required to allow controllable thermal isolation of the refrigerant. This heat switch is fabricated from a superconducting material with a low critical magnetic field (often aluminium). With a magnetic field above $B_{c}$ applied to the heat switch, the conduction electrons are free to transport heat though it, however with the field below $B_{c}$ there is negligible thermal conductivity [27] at low temperatures due to the small populations of free electrons and phonons. The paired electrons making up the superconducting state are condensed into the ground state, thus have no entropy and can carry no heat. The magnetic field setting the state of the heat switch can be produced either using a dedicated control superconducting magnet, or using the fringing field of the main solenoid [28].

The choice of material, quantity of refrigerant and the magnetic field ranges to be used are dependent on the desired final temperature and the heat leak into the cooled system. For a perfectly adiabatic process, the minimum temperature is limited by the spontaneous magnetic ordering temperature or by the effective internal magnetic field $b$, after which point no further cooling will occur. There may also be undesirable phase transitions which further limit the minimum temperature and field to above what would otherwise be expected. In practice the demagnetisation will not be perfectly adiabatic due to the presence of some heat leak, hence the minimum temperature is usually limited by the available nuclear heat capacity. This can be calculated from the result in Equation 2.10 using

$$
C_{n}=\left.T_{n} \frac{\partial S}{\partial T_{n}}\right|_{B}
$$

which gives

$$
C_{n}=\frac{\lambda_{n} B^{2}}{\mu_{0} T_{n}^{2}}
$$

To use this result in calculating the available cooling power $\dot{Q}_{n}$, we first define $\tau_{1}$, the spin-lattice relaxation time:

$$
\frac{\mathrm{d}}{\mathrm{d} t}\left(\frac{1}{T_{n}}\right)=-\frac{1}{\tau_{1}}\left(\frac{1}{T_{n}}-\frac{1}{T_{e}}\right)
$$

This is the characteristic time for the nuclear spin temperature $T_{n}$ to reach thermal equilibrium with the conduction electrons which are at temperature $T_{e}$. Using the Korringa law [29], we can relate the spin-lattice relaxation time to a material 
specific constant $\kappa$ (the Korringa constant) and the electron temperature:

$$
\tau_{1} T_{e}=\kappa
$$

This gives us a more useful form of Equation 2.15:

$$
\frac{\mathrm{d} T_{n}}{\mathrm{~d} t}=\frac{T_{n}\left(T_{e}-T_{n}\right)}{\kappa}
$$

Using the definition of heat capacity, and noting that $\mathrm{d} C_{n} / \mathrm{d} t=0$, we have

$$
\dot{Q}_{n}=C_{n} \dot{T}_{n}=\frac{\lambda_{n}\left(B_{e x t}^{2}+b^{2}\right)}{\mu_{0} \kappa T_{n}}\left(T_{e}-T_{n}\right),
$$

where we have substituted the nuclear heat capacity from Equation 2.14 and expanded the total magnetic field into its external and effective internal components. With knowledge of the anticipated heat leak into the refrigerated system, this equation can be used to assist with the selection of the appropriate nuclear refrigerant. In addition to finding a material which has the optimal combination of parameters such that $\dot{Q}_{n}$ is maximised there are additional considerations, such as the presence of superconductivity, nuclear quadrupole moments and electronic ordering, that can have a strong negative effect on the values in this equation at particular points during a demagnetisation [30]. The end result of this is that there are very few materials that are usable at all, and most that can be used are far from optimal. This leaves us with the two workhorses of nuclear refrigeration: $\mathrm{Cu}$ and $\mathrm{PrNi}_{5}$, with $\mathrm{In}, \mathrm{Pt}$ and $\mathrm{Tl}$ being more exotic choices investigated during the development of the technique [25].

Copper is a popular choice thanks to its relatively large nuclear Curie constant, absence of superconductivity or other phase transitions, and high thermal conductivity. The ease of manufacture of copper components and its readily available nature is also highly attractive. Additionally, the internal magnetic field from the interactions of the nuclear magnetic moments is very small, $b=0.36 \mathrm{mT}$, allowing demagnetisation to a low field and hence the lowest temperatures. The initial experiments in the demagnetisation of copper nuclei produced nuclear temperatures of $15 \mu \mathrm{K}$ with the copper electrons and lattice held warm at dilution refrigerator temperature [31]. This was possible due to copper's large Korringa constant $(\kappa=1.21 \mathrm{Ks})$ which represents weak coupling between the nuclei and electrons. While this, in conjunction with copper's relatively high nuclear heat capacity, is an advantage for these nuclear cooling experiments, it is a significant disadvantage for general refrigeration as any items to be cooled must, at the very least, couple through the copper electrons. A further disadvantage is the chal- 
lenging starting conditions that must be obtained to achieve a significant energy reduction: at a starting temperature of $T_{i}=10 \mathrm{mK}$, ramping the magnetic field from zero to $B_{i}=8 \mathrm{~T}$ gives an entropy reduction of only $9 \%$ after precooling [23].

The Van-Vleck paramagnet $\mathrm{PrNi}_{5}$ overcomes some of these issues by having a very small Korringa constant with upper bound $\kappa<10 \mu \mathrm{Ks}$ [32]. It also has a very high nuclear Curie constant, some 440 times greater than that of copper. These effects provide a huge nuclear heat capacity that is accessible through the strongly coupled conduction electrons, allowing whole refrigeration platforms to be designed around it [33]. Base temperatures with this material are however limited by the internal magnetic field $b=66 \mathrm{mT}$ and spontaneous magnetic ordering temperature $T_{c}=400 \mu \mathrm{K}$ [34] (c.f. $b=0.36 \mathrm{mT} \& T_{c}=50 \mathrm{nK}$ for $\mathrm{Cu}$ [35]). The poor thermal conductivity of the material itself (comparable to that of brass at low temperatures [36]) also limits the ultimate temperatures of attached samples. Depending on the balance of the heat leaks and available cooling power, together with the temperature targeted, these aspects may not be significant, however the difficulty in obtaining and working with the material make its use somewhat unattractive [37].

\subsection{Coulomb Blockade Thermometry}

A CBT is a low temperature, nanoelectronic thermometer which measures the temperature of its internal electrons. Since it is the electrons within nanoelectronic devices that we are aiming to cool for this investigation, the CBT is an ideal device for proof of concept tests of the techniques developed in this thesis. In this section, a basic description of the principles and background of CBTs is given in Subsection 2.2.1. This is followed by a discussion of the theory concerning CBT operation in Subsection 2.2.2 and finally by a description of the use of CBTs in practice in Subsection 2.2.3.

\subsubsection{Outline}

The CBT is a nanoelectronic device first described in 1994 [38]. In its simplest form it consists of a pair of tunnel junctions creating a small metallic island between them, denoted a NININ (normal metal-insulator-normal metal-insulator-normal metal) structure, the electrical conductance of which is measured. From the electrical conductance, the temperature of the electrons in the device structure can be deduced using only fundamental physical constants. This makes the device a primary thermometer, i.e. it provides an accurate temperature reading without requiring calibration. 
The CBT operates, as its name suggests, using a particular regime of the Coulomb blockade effect. Put briefly, Coulomb blockade occurs at low temperatures when the available thermal energy is less than the energy required to charge the island. The discreteness of electron charge means that the island must be charged by at least $e$, hence setting a minimum amount of energy, below which no current can flow through the structure [39]. A single metallic island has a stray capacitance to ground $C_{0}$ and the tunnel junctions also each contribute a capacitance $C_{J}$, leading to a total capacitance of the island $C_{\Sigma}=C_{0}+2 C_{J}$. A capacitor of capacitance $C$ containing charge $Q$ holds electrostatic energy $Q^{2} /(2 C)$. Hence, starting from a neutrally charged island, if we add an additional electron (or remove one), we must supply energy

$$
E_{c}=\frac{e^{2}}{2 C_{\Sigma}}
$$

For this to produce any noticeable effect this quantity, the charging energy, must be at least comparable with the thermal energy $k_{B} T_{e}$ held by the electrons. To achieve this at accessible temperatures the physical size of the metallic island, in addition to the size of the tunnel junctions, is controlled such that $C_{\Sigma}$ is small. For the construction of SETs, the capacitance is chosen such that it is in the strong Coulomb blockade regime $E_{c} \gg k_{B} T_{e}$, where the probability of any single electron having sufficient energy to overcome the blockade is negligible. The CBT, however, operates in an intermediate regime where the charging energy and thermal energy are comparable. The conductance curves for the three possible regimes the metallic island can be under are sketched schematically in Figure 2.3. Figure 2.3a shows the usual situation where there is no Coulomb blockade, Figure 2.3b shows the weak Coulomb blockade regime where Coulomb blockade thermometry can take place, and Figure 2.3c shows the strong Coulomb blockade regime used for SETs and quantum dots.

As shown in Figure 2.3b, in the weak Coulomb blockade regime the full width at half maximum (FWHM) voltage has a linear relation to temperature given by

$$
V_{1 / 2} \approx 2 \times 5.439 k_{B} T_{e} / e
$$

for $V_{1 / 2}$ the FWHM of the dip seen in a plot of island conductance against the applied bias, $k_{B}$ the Boltzmann constant, $T_{e}$ the temperature of the electrons on the island and $e$ the electronic charge. The numerical constant in this equation solely arises from successive mathematical approximations and does not contain any empirical parameters [40]. The only other constants in the equation are fundamental physical constants, hence showing the primary nature of thermometry performed using such a device. 


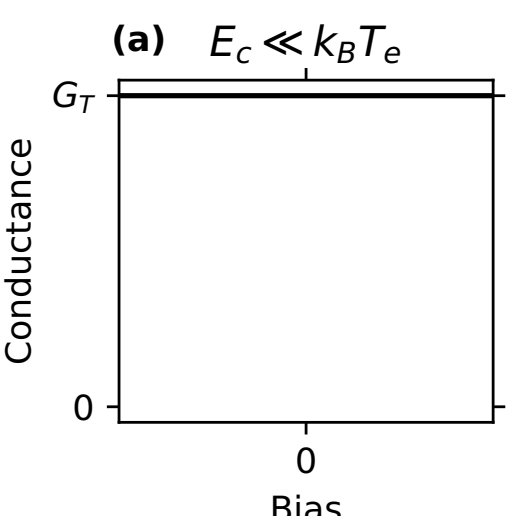

Bias

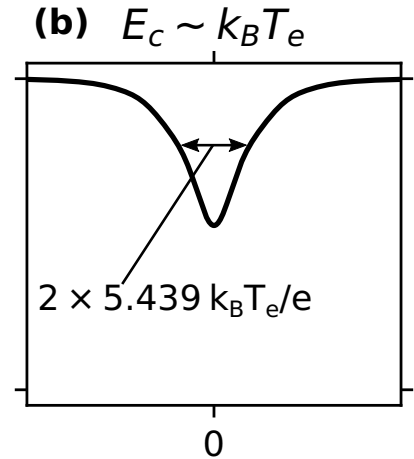

Bias

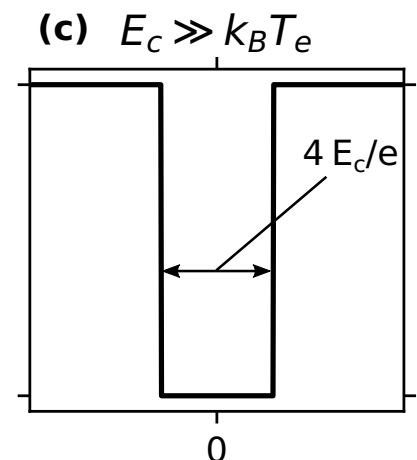

Bias

Figure 2.3: Coulomb Blockade on a Single Metallic Island. The plots show schematically the conductance of a single metallic island with charging energy $E_{c}$ situated between two tunnel junctions, each with tunnel resistance $R_{T}=\left(2 G_{T}\right)^{-1}$. The electron temperature is $T_{e}$. Panel (a) shows small $E_{c}$, producing no Coulomb blockade effects. Panel (b) shows weak Coulomb blockade, the regime of operation for a CBT where the dip width is directly proportional to temperature. Panel (c) shows large $E_{c}$ causing full Coulomb blockade.

As well as the different Coulomb blockade regime used in CBTs, as compared to SETs, there is a further difference in the device construction. A SET has a third electrode which acts as a gate and is capacitively coupled to the island between the tunnel junctions [41]. The effect of this gate is to control the island background charge. If the background charge is set such that it completely compensates the charge from the electrons on the island, then the Coulomb blockade is lifted and an electron can tunnel on or off the island. Alternatively, the background charge can be chosen to produce the strongest blockade possible (i.e. maximal bias required to cause current flow), or any point in between. A CBT has no such electrode, so the background charge on each island takes a random value from a uniform distribution. For a CBT operating well within the weak Coulomb blockade regime, the change in the conductance characteristic arising from this is negligible [40] and in any case is averaged out by a chain of islands.

\subsubsection{Orthodox Theory of Single Electron Tunnelling}

Most practical CBTs use more than just a single island since higher order tunnelling phenomena result in significant erroneous temperature readings [42, 43] for single island devices. Using a long chain of $N$ tunnel junctions in series, hence producing $N-1$ islands, means that these effects are both suppressed and averaged out across the chain, the inner islands are shielded from the tunnelling of hot electrons from the leads by the outer islands, and the voltage noise is divided across the 
chain. A long chain of a few tens of junctions results in a high resistance that is difficult to measure. Hence two-dimensional arrays are used, where there are multiple parallel strings of islands in series, allowing the measurement of a more reasonable resistance in the $\sim 10 \mathrm{k} \Omega$ range.

For a tunnel junction array operating in the weak Coulomb blockade regime, the conductance as a function of temperature can be derived by starting from the 'orthodox single electron theory' [44]. In this we calculate the difference in free energy $\Delta F$ before and after an individual electron tunnelling event. Then, the tunnelling rate for this particular event can be calculated using Fermi's golden rule:

$$
\Gamma_{i \rightarrow j}(\Delta F)=\frac{2 \pi}{\hbar}\left|M_{i j}\right|^{2} \delta\left(E_{i}-E_{j}-\Delta F\right),
$$

where $i$ and $j$ refer to the states of the system before and after the transition, respectively, hence $E_{i}$ and $E_{j}$ are the initial and final energies of the tunnelling electron and $M_{i j}$ the matrix element for the two states, giving the transmission probability. The total rate is then calculated by summing the rates for all possible initial and final state combinations, with each rate being weighted by the probability of the initial state being occupied and the final state being unoccupied. The probability of a state being occupied is given by the Fermi-Dirac distribution

$$
f(E)=\frac{1}{1+\exp \left(\frac{E-E_{F}}{k_{B} T}\right)},
$$

for a state of energy $E$ on a node with Fermi energy $E_{F}$. Here the term 'node' is used to refer to a conductor, so includes the islands between the tunnel junctions and the electrodes on the end of the chains. The total rate is therefore

$$
\Gamma(\Delta F)=\frac{2 \pi}{\hbar} \sum_{i} \sum_{j}\left|M_{i j}\right|^{2} f\left(E_{i}\right)\left(1-f\left(E_{j}\right)\right) \delta\left(E_{i}-E_{j}-\Delta F\right) .
$$

These sums can be replaced with an double integral by inserting the initial and final densities of states, $D_{i}$ and $D_{j}$, respectively. The integrals are then performed over all initial and final energies in the conduction band on the initial and final nodes:

$$
\Gamma(\Delta F)=\frac{2 \pi}{\hbar} \int_{-\infty}^{\infty} \int_{-\infty}^{\infty} D_{i} D_{f}\left|M_{i j}\right|^{2} f\left(E_{i}\right)\left(1-f\left(E_{j}\right)\right) \delta\left(E_{i}-E_{j}-\Delta F\right) \mathrm{d} E_{i} \mathrm{~d} E_{j}
$$

The transmission coefficient through the tunnel junction, matrix element $M_{i j}$, is assumed to be independent of what exactly the states $i$ and $j$ are, and is then written in terms of a new phenomenological parameter $R_{T}$, the tunnelling resistance, 
which is defined by

$$
R_{T}=\frac{V}{I}
$$

the simple Ohmic resistance observed in the current-voltage characteristic of a single tunnel junction. This is related to the matrix element by [45]

$$
R_{T}=\frac{\hbar}{2 \pi e^{2}\left|M_{i j}\right|^{2} D_{i} D_{f}}
$$

Substituting for $\left|M_{i j}\right|$ in Equation 2.24 and moving constants to outside the integrand, the total rate simplifies to

$$
\Gamma(\Delta F)=\frac{1}{e^{2} R_{T}} \int_{-\infty}^{\infty} \int_{-\infty}^{\infty} f\left(E_{i}\right)\left(1-f\left(E_{j}\right)\right) \delta\left(E_{i}-E_{j}-\Delta F\right) \mathrm{d} E_{i} \mathrm{~d} E_{j} .
$$

The delta function means that the only contribution from one of the integrals is when $E_{i}-E_{j}-\Delta F=0$. Hence one integral can be evaluated by setting $E_{j}=E_{i}-\Delta F$ and relabelling $E_{i} \rightarrow E$. This gives

$$
\Gamma(\Delta F)=\frac{1}{e^{2} R_{T}} \int_{-\infty}^{\infty} f(E)(1-f(E-\Delta F)) \mathrm{d} E,
$$

which evaluates to

$$
\Gamma(\Delta F)=\frac{\Delta F}{e^{2} R_{T}\left[\exp \left(\frac{\Delta F}{k_{B} T}\right)-1\right]} .
$$

This relies on the assumption that the tunnel barriers are 'sufficiently opaque' that the electrons are localised on islands or the connecting leads, not delocalised throughout the chain. This condition, known as the 'weak tunnelling limit', can be derived using the Heisenberg uncertainty principle and requires that

$$
R_{T}>\frac{h}{e^{2}}=R_{Q} \approx 26 \mathrm{k} \Omega
$$

hence explaining the necessarily large resistances encountered if a 1-dimensional series chain of tunnel junctions is used to create a CBT rather than a 2-dimensional array.

The charge distribution across the device can be described by the set $\left\{n_{i}\right\}$, which gives the number of excess electrons $n_{i}$ on each island $i$. Each unique charge distribution will give a different set of tunnelling rates, each of which can be calculated by considering the change in electrostatic energy between the initial charge distribution and that after the tunnelling event. This done by noting that the net charge on the $i^{\text {th }}$ island is given by $-n_{i} e$, and hence a vector $\boldsymbol{q}$ listing the charges on all islands can be created from the initial charge distribution $\left\{n_{i}\right\}$. This 
can then be repeated for the final charge distribution, which differs only by the movement of a single electron to an adjacent island through a particular tunnel junction in a particular direction.

From the charge vectors, the electrostatic energies before and after a single tunnelling event, and hence the free energy change $\Delta F$, are calculated using a capacitance matrix $\boldsymbol{C}$ which describes the capacitances between all nodes (islands, electrodes, grounds and, optionally, background charges) in the system [46]. With this, we can say that the free energy is a function of the initial $\left(\left\{n_{i}\right\}\right)$ and final $\left(\left\{n_{f}\right\}\right)$ charge distributions, but with no explicit dependence on the particular tunnel junction involved since all the junctions here are assumed to be identical [47]:

$$
\Delta F=\Delta \mathrm{F}\left(\left\{n_{i}\right\},\left\{n_{f}\right\}\right)=\Delta \mathrm{F}_{k}^{ \pm}\left(\left\{n_{i}\right\}\right)
$$

The second equality here follows for a single tunnelling event at the $k^{\text {th }}$ tunnel junction in the positive or negative direction $( \pm)$, since the final charge distribution is readily calculated from the initial distribution (given the direction of the tunnelling event and the junction at which it occurs). Therefore, by inserting Equation 2.31 into Equation 2.29 we define the rate function

$$
\Gamma_{k}^{ \pm}\left(\left\{n_{i}\right\}\right)=\Gamma\left(\Delta \mathrm{F}_{k}^{ \pm}\left(\left\{n_{i}\right\}\right)\right)
$$

which gives the tunnelling rate through the $k^{\text {th }}$ tunnel junction, in the positive or negative direction $( \pm)$, as a function of the initial overall charge distribution $\left(\left\{n_{i}\right\}\right)$.

Each tunnelling rate can then be weighted by the probability of the charge configuration $\sigma\left(\left\{n_{i}\right\}\right)$ that it is the result of. These probabilities can be obtained by requiring that in the steady state there is no build up of charge at any node. This leads to a tunnel rate master equation, which for the single island case reads [38]

$$
\begin{aligned}
\dot{\sigma}(n) & =\left[\Gamma_{1}^{+}(n-1)+\Gamma_{2}^{-}(n-1)\right] \sigma(n-1) \\
& -\left[\Gamma_{1}^{+}(n)+\Gamma_{1}^{-}(n)+\Gamma_{2}^{+}(n)+\Gamma_{2}^{-}(n)\right] \sigma(n) \\
& +\left[\Gamma_{1}^{-}(n+1)+\Gamma_{2}^{+}(n+1)\right] \sigma(n+1)=0,
\end{aligned}
$$

where $\left\{n_{i}\right\}$ has been replaced by $n$, the number of excess electrons on the single island. The probabilities for the possible charge distributions can be found by solving this, or a more general version for multiple islands [40].

With this, the net current through the $k^{\text {th }}$ tunnel junction, which must necessarily be equal to the current though the other junctions in series to avoid a build up of 
charge, can be found by summing over all the possible charge configurations:

$$
I_{k}=-e \sum_{\left\{n_{i}\right\}} \sigma\left(\left\{n_{i}\right\}\right)\left[\Gamma_{k}^{+}\left(\left\{n_{i}\right\}\right)-\Gamma_{k}^{-}\left(\left\{n_{i}\right\}\right)\right]
$$

This can be calculated for any value of $k$ and then the overall differential conductance of the device calculated from

$$
G=P \frac{\mathrm{d} I_{j}}{\mathrm{~d} V}
$$

for $P$ the number of parallel island chains and $V$ the bias applied across the device. From this point, the overall conductance can either be modelled numerically, or we can make an approximation by assuming high temperatures:

$$
k_{B} T \gg \frac{e^{2}}{C_{\Sigma}}
$$

With this assumption, after expanding the results of Equation 2.34 to first order in $E_{c} / k_{B} T$, Equation 2.35 eventually gives the result [40]

$$
\frac{G}{G_{T}}=1-\frac{E_{c}}{k_{B} T} \frac{v_{N} \sinh \left(v_{N}\right)-4 \sinh ^{2}\left(v_{N} / 2\right)}{8 \sinh ^{4}\left(v_{N} / 2\right)},
$$

where we have defined

$$
E_{c} \equiv \frac{N-1}{N} \frac{e^{2}}{C_{\Sigma}}
$$

and

$$
v_{N} \equiv \frac{e V}{N k_{B} T} .
$$

This describes the ratio between the conductance of an island chain at bias $V$ as a fraction of the high bias, asymptotic conductance $G_{T}$. Approximating this further then gives us a version of Equation 2.20 valid for chains of islands [47]:

$$
V_{1 / 2} \approx 5.439 N k_{B} T / e,
$$

and we have the depth of the dip in the conductance curve at zero bias:

$$
\frac{\Delta G}{G_{T}} \approx \frac{E_{c}}{6 k_{B} T}
$$

Equations 2.40 and 2.41 have been confirmed experimentally [48] to have acceptable accuracy for $k_{B} T / E_{c}>3$. If instead, $G$ is expanded to third order in 
$E_{c} / k_{B} T$ [40], we find a more accurate version of Equation 2.41

$$
\frac{\Delta G}{G_{T}} \approx \frac{1}{6}\left(\frac{E_{c}}{k_{B} T}\right)+\frac{1}{60}\left(\frac{E_{c}}{k_{B} T}\right)^{2}+\frac{1}{630}\left(\frac{E_{c}}{k_{B} T}\right)^{3}
$$

and a more accurate version of Equation 2.40

$$
V_{1 / 2} \approx 5.439 \frac{N k_{B} T}{e}\left(1+0.3921 \frac{\Delta G}{G_{T}}\right) .
$$

This allows primary thermometry to be retained at a $2.5 \%$ tolerance for $k_{B} T / E_{c}>$ 0.4, the intermediate Coulomb blockade regime [48]. Similar accuracy can alternatively be maintained by using the numerical full tunnelling model [49]. It is also possible to perform a Markov chain Monte Carlo (MCMC) simulation of the effect of background charges along the chain to allow measurements at $k_{B} T / E_{c}=0.2$ to be made with a $2 \%$ temperature variation across the $3 \sigma$ confidence interval [50].

The capacitance of the CBT tunnel junctions can be controlled though their dimensions, and similarly the stray capacitance is set by the island dimensions. This allows the operation range of CBTs to be chosen. To date, CBTs have been shown to operate up to $60 \mathrm{~K}$ [51] to assist with the redefinition of the Kelvin based on fundamental physical constants and to provide practical thermometry with accepted accuracy [52]. Other CBTs have been shown to operate down to $3.7 \mathrm{mK}$ to aid the development of new techniques for low temperature nanoelectronics [49].

\subsubsection{Practical Measurements}

Practical primary thermometry can be performed with a CBT sensor by taking measurements of the full conductance curve, and then analysing it according to the temperature range the CBT is operating in. The overall practical operation range of a CBT can be approximately stated as $0.2<k_{B} T / E_{c}<20$. For $3<k_{B} T / E_{c}<$ 20 , the electron temperature can be accurately found by using the FWHM of the conductance dip with Equation 2.40 [47]. For $0.4<k_{B} T / E_{c}<3$, accurate electron temperature measurements require the use of either the more accurate Equations 2.42 and 2.43 [48], or the numerical full tunnelling model [49]. Finally for $0.2<k_{B} T / E_{c}<0.4$, the effect of the island background charges starts to become important and must be simulated in order to obtain an accurate temperature for the most likely background charge distribution. This simulation also allows one to estimate the uncertainty arising from the distortion of the conductance characteristic as a result of the other possible distributions [40, 50]. The upper limit of this range, $k_{B} T / E_{c} \approx 20$, is set by the fact that above this the magnitude of the conductance dip [40] becomes small $(<1 \%)$, making measurements difficult 
and slow.

For faster thermometry measurements, subject to the provisions above regarding temperature ranges, the CBT can be operated in a pseudo-secondary or selfcalibrated mode. In this configuration only a single data point, the CBT zero bias conductance, needs to be measured for each temperature datum instead of the full conductance curve, making measurements many times faster. For monitoring a dynamic cooling process, using the device in this way is essential for achieving a satisfactory time resolution. This mode is enabled by first measuring several conductance curves, distributed across the sensor temperature operation range, then fitting a full tunnelling model to the measured data [40, 49]. This allows us to extract the total capacitance per island $C_{\Sigma}$ and tunnel resistance $R_{T}$ from the fitting parameters. Using these values Equation 2.41, or an appropriately expanded or modelled version thereof, can then be used to extract the temperature from the zero bias conductance.

\subsection{On-Chip Refrigeration}

On-chip cooling refers to a few cooling techniques, operating in different temperature regimes, where the refrigerant is of a comparable size to the circuit elements on some micro- or nanoelectronic device. This section introduces and motivates the field in Subsection 2.3.1, and then explores the techniques used, including the new one explored in this thesis, in Subsection 2.3.2.

\subsubsection{Motivation and Principles}

Some forms of on-chip cooling are motivated by the potential replacement of bulky, expensive and complicated to operate cooling solutions such as dilution refrigerators. These techniques are not motivated by the potential discovery of new physical effects or improved operation of devices at new, lower temperatures as they simply aim to replicate already achievable temperatures in a different way.

The other motivation, which we are following here, is to use on-chip cooling to reach electron temperatures in nanoelectronic devices that are otherwise unreachable with traditional techniques. For many physical effects being explored in nanoelectronic devices, we require the thermal energy of the electrons to be less than the characteristic energy of the desired effect, hence low electron temperatures are needed. Traditional cooling techniques rely on passively cooling the electrons via the electrically insulating device substrate, hence cooling the device phonons which in turn cool the electrons via electron-phonon coupling (see Figure 2.4). For 


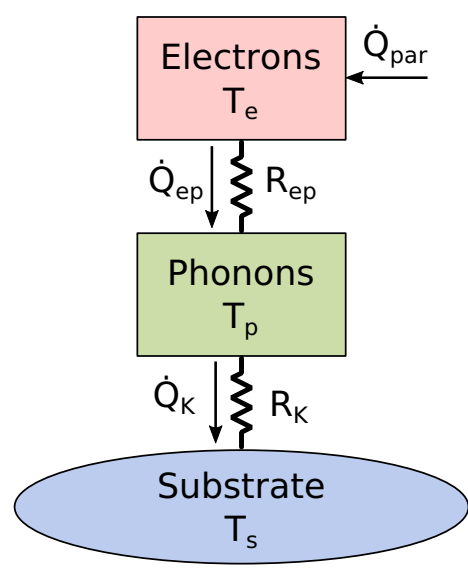

Figure 2.4: Traditional Passive Cooling of a Nanoelectronic Device. A standard cooling technique relies on the electron phonon coupling transferring heat, $\dot{Q}_{\mathrm{ep}}$, from the device electrons at $T_{e}$ to the device phonons at $T_{p}$. This heat in turn flows to the substrate at temperature $T_{s}$ via the Kapitza resistance $R_{K}$. The minimum electron temperature occurs when the electrons are in thermal equilibrium, with the parasitic heat leak $\dot{Q}_{\text {par }}$ balanced by the electron phonon coupling.

macroscopic structures, or nanostructures above, say $\sim 100 \mathrm{mK}$, these techniques result in negligible temperature differences between the electrons and their host lattice. For lower temperatures, however, the temperatures diverge [13, 53]. This occurs due to the weak electron-phonon coupling at low temperatures. The power transferred from the electrons to the phonons by this coupling is given by [53]

$$
\dot{Q}_{\mathrm{ep}}=\Sigma V\left(T_{e}^{n}-T_{p}^{n}\right)
$$

where $\Sigma$ is a material specific coupling constant, $V$ the volume over which thermalisation of the electrons and phonons can occur and $T_{p}$ and $T_{e}$ are the phonon and electron temperatures, respectively. The value of $n$ is commonly accepted to be 5 in many materials $[23,53,54]$, so the equation is often stated as

$$
\dot{Q}_{\mathrm{ep}}=\Sigma V\left(T_{e}^{5}-T_{p}^{5}\right)
$$

however, the power has been experimentally observed to take other values $2<$ $n \leq 5$, particularly in systems confined to fewer dimensions $[49,55]$. In any case, the heat flow is strongly temperature dependent, leading to weak low-temperature coupling for nanoelectronic devices where $V$ is, by definition, small.

An additional complication lies in the thermal boundary resistance between the device itself and the substrate it is mounted on, and at all further connections between different materials where heat is transported by phonons. This thermal 
resistance is known as the Kapitza resistance, and is defined as [56]

$$
R_{K}=\frac{\dot{Q}}{\Delta T}
$$

for $\dot{Q}$ the rate of heat transport and $\Delta T$ the temperature difference across the interface. This resistance can be particularly problematic for the interface between the liquid helium in the MXC of a dilution refrigerator and the metal heatsinking used for attaching devices, where it arises due to the acoustic mismatch between the phonons on each side of the boundary. There are, nevertheless, well tested techniques used for minimising this resistance through the use of sintered silver in contact with the helium mixture [57].

For typical low temperature nanoelectronic measurements, the device is brought into contact with the MXC plate of a dilution refrigerator, that is a metallic plate in good thermal contact with the mixing chamber where the ${ }^{3} \mathrm{He}-{ }^{4} \mathrm{He}$ dilution takes place. The base temperature of practical dilution refrigerators varies from $300 \mathrm{mK}$ (above which the simpler ${ }^{3} \mathrm{He}$ evaporation cryostat would be used) to $1.75 \mathrm{mK}$ for cutting edge machines [6]. More common temperatures are of the order $10 \mathrm{mK}$, which are easily reachable in readily available and simple to operate commercial machines [7]. Despite these low MXC temperatures, which the device phonon temperature will get close to, in most situations the electrons will remain significantly overheated, up to $\sim 100 \mathrm{mK}$ in some cases [14]. Indeed, with particular effort to ensure a low noise environment in a low temperature dilution refrigerator, electron temperatures around $\approx 10 \mathrm{mK}$ can be achieved [58, 59], however, electron temperatures lower than this are rare [60]. This largely remains true even if the chip is attached to a nuclear demagnetisation stage cooled to $\sim 100 \mu \mathrm{K}$ or lower.

This overheating is the result of thermal equilibrium being reached at an elevated temperature due to the strong temperature dependence of the electron-phonon coupling. The source of the heat input is impossible to completely pinpoint, but can arise from electrical noise [61], electromagnetic radiation [62, 63] or vibration (particularly relevant in a magnetic field, where vibrations cause eddy current heating [64]). It has previously been attempted to overcome these issues by reducing heat leaks to a minimum and maximising the coupling. Heat leaks can be reduced using vibration isolation, radiation shielding and extensive noise filtering on the connecting wiring [65]. The coupling can be improved by using large metallic structures on the device, which increases $V$ in Equation 2.45. The material used can also be selected in order to maximise $\Sigma$, for example gold has the largest known value of $2.4 \mathrm{GWK}^{-5} \mathrm{~m}^{-3}$ [20]. A method for combining these two techniques involves cooling a device with sintered thermalisation blocks immersed in a very 
cold liquid helium bath, either as part of the dilution refrigerator's MXC [49] or as a separate ${ }^{3} \mathrm{He}$ cell [66-68]. This provides a very low temperature environment, shielding the device from heat leaks. It also ensures the phonon temperature is as close as possible to the refrigerator's base temperature by limiting the effects of Kapitza resistance. The lowest temperature obtained using this technique is $3.7 \mathrm{mK}$ with a heat leak into each island on the CBT of $\geq 300 \mathrm{aW}$ [49].

In most cases, however, the minimum temperatures achievable will not be significantly improved by increasing $\Sigma$ and $V$, since the dependence of $T_{e}$ on these parameters is weak, as can be seen by the $1 / 5$ power in a rearrangement of Equation 2.45:

$$
T_{e}=\left(\frac{\dot{Q}_{\mathrm{ep}}}{\Sigma V}+T_{p}^{5}\right)^{1 / 5}
$$

\subsubsection{Techniques}

Some types of of on-chip cooling are electronic in nature [69]. One implementation is based on a normal metal-insulator-superconductor (NIS) junction. Here, the energy gap in the superconductor blocks the tunnelling of low energy electrons. Hence if starting from a reasonably low temperature, say $300 \mathrm{mK}$ achieved using a ${ }^{3} \mathrm{He}$ evaporation cryostat, and biasing the junction at slightly less than $\Delta / e$ (for $\Delta$ the superconducting gap), only the highest energy electrons in the distribution are able to tunnel through the barrier. This filters off the hot electrons onto the superconductor, leading to a reduced average energy hence a lower electron temperature on the normal metal section [70]. When arranged into a symmetric configuration with a junction on each side of the normal metal island we have a SINIS (Superconductor-Insulator-Normal metal-Insulator-Superconductor) structure. These structures provide a significantly increased cooling power as, while high energy electrons are removed at one junction, they are replaced with low energy electrons (or equivalently, high energy holes leave) at the other. These coolers are effective in cooling the electrons from $\approx 300 \mathrm{mK}$ to $\approx 100 \mathrm{mK}$ [71], i.e. from the base temperature of a ${ }^{3} \mathrm{He}$ evaporation cryostat to well within the dilution refrigerator temperature range.

A variation of this junction cooler design replaces the normal metal with a superconductor of different gap energy, referred to as S' giving a SIS' junction. Their method of operation is analogous to the NIS coolers but with a different optimal bias voltage. A device of this structure has been used to cool from $1 \mathrm{~K}$ to $400 \mathrm{mK}$ [72], so from the base temperature of a ${ }^{4} \mathrm{He}$ evaporation cryostat to just above that of a ${ }^{3} \mathrm{He}$ cryostat. Another possible variation is the substitution of the normal-metal with a heavily doped semiconductor. Again, the operation 


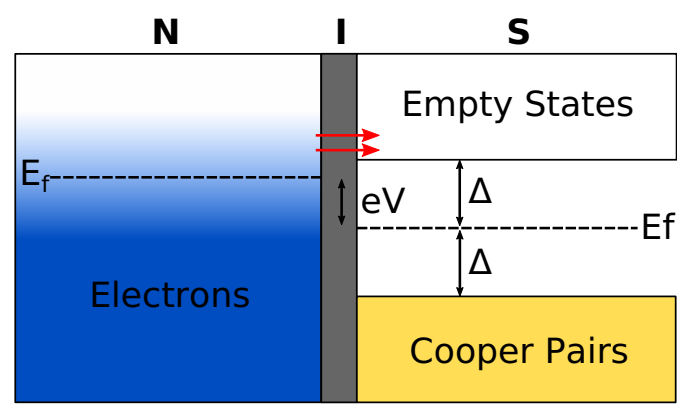

Figure 2.5: Schematic energy diagram of an NIS cooler. The applied bias $e V<\Delta$ raises the Fermi level $E_{f}$ of the normal metal $(\mathbf{N})$ above that in the superconductor $(\mathbf{S})$. This allows only the hottest electrons to tunnel through the insulator (I), filtering them off into the superconductor and hence cooling the normal metal.

principle is analogous to the normal-metal case, however, the fabrication process is simplified as the resulting Schottky barriers between the semiconductor and superconductors act as the tunnel junctions [73], meaning a discrete insulating layer does not have to be made. The electron-phonon coupling in a semiconductor is weaker than that in a metal, reducing the heat leak to the electrons from the surrounding warm lattice and allowing cooling from $300 \mathrm{mK}$ down to $174 \mathrm{mK}$ [74].

Another type of electronic on-chip cooler is the quantum-dot cooler. Quantum-dot coolers are formed of an isolated island separated from a pair of electrodes by two quantum dots. A small direct current (DC) bias is applied across the structure and the quantum dot energies are set via gates such that one is slightly above and one is slightly below the chemical potential of the island. As a current flows through the structure, the two quantum dots force the electrons leaving the island through one quantum dot to be at a higher energy than the electrons being injected onto the island through the other quantum dot. This leads to a net decrease in energy and hence cooling $[75,76]$. This technique was demonstrated experimentally on a two-dimensional electron gas sample where the island electrons were cooled from $280 \mathrm{mK}$ to $187 \mathrm{mK}$ [77].

These electronic coolers, by designing the sample to minimise heating from the surroundings, can be extended to cool the surrounding lattice via the electron-phonon coupling operating in the opposite direction to in passive cooling [69]. Some have also been extended to enable practical use as an alternative to a dilution refrigerator [78, 79], and the minimum temperatures reachable with these techniques has been steadily falling to as low as $30 \mathrm{mK}$ as the junction technology has improved [80-82]. Progress towards the development of a viable dilution refrigerator replacement based on this technique is therefore promising, however, it is not yet at the point of being usable for reaching new low electron temperatures in devices. 
The main purpose of this study is to investigate a new type of on-chip cooler using adiabatic demagnetisation with the refrigerant located directly on the chip. At the start of this study, there were no descriptions of this technique in the literature. Shortly after the start of this study, an investigation into a SET fabricated from aluminium doped with manganese was published [83]. Aluminium is a common material for the fabrication of structures containing tunnel junctions [84] due to the high-quality, thin insulating layers that can easily be made through controlled oxidation of evaporated aluminium structures [69, 85]. Aluminium superconducts at $T_{c}=1.19 \mathrm{~K}$ [37] which presents a problem for the production of a normal-metal SET intended to operate below this. One solution is to dope the aluminium with a magnetic impurity such as manganese to suppress the superconductivity [86]. As well as suppressing the superconductivity, however, the manganese atoms make the structure paramagnetic and and hence allowing demagnetisation cooling. This allowed the cooling of the SET from the base temperature of a ${ }^{3} \mathrm{He}$ cryostat $(\approx$ $300 \mathrm{mK}$ ) to $140 \mathrm{mK}$ [83].

On-chip demagnetisation cooling can also be implemented by applying magnetic refrigerant directly on top of the metallic conductors on the nanoelectronic device. For this to work, the refrigerant must be in electrical contact with the circuit elements, otherwise the electron cooling must once again proceed via the weak electron-phonon coupling, as would be the case if a traditional elaborate demagnetisation stage [87] was used. The use of on-chip magnetic refrigerant is the approach investigated in this thesis. 


\section{Chapter 3}

\section{On-Chip Demagnetisation Cooling on a Cryogen-Free Dilution Refrigerator}

This chapter describes a proof of concept study of on-chip demagnetisation performed on a CBT with additional copper thermalisation blocks which act as the on-chip refrigerant. CBTs are particularly well suited to this purpose because they provide primary thermometry of their own internal electron temperature, which is what we are aiming to reduce. In addition, their structure is similar to SETs which is one example of a device that one may wish to apply this cooling technique to. The results of this study were published in [88], which was drafted by the author of this thesis, and can be summarised as the electrons being cooled from $9 \mathrm{mK}$ to below $5 \mathrm{mK}$ for $1200 \mathrm{~s}$.

In this chapter, Section 3.1 describes the design and fabrication of the CBT used for this part of the investigation. The installation of the CBT in the dry dilution refrigerator in addition to the electrical measurement scheme are described in Section 3.2. This is followed by the results from characterising the CBT in Section 3.3 and the on-chip cooling results in Section 3.4. Finally, we conclude in Section 3.5.

\subsection{Coulomb Blockade Thermometer Device}

The CBT used in this study was fabricated by collaborators at VTT, Technical Research Centre of Finland, as a variation of the devices used in an earlier study into reducing the electron temperature through immersion cooling [49]. The device was fabricated on an undoped silicon wafer of diameter $150 \mathrm{~mm}$ (nominally 6-inch) 


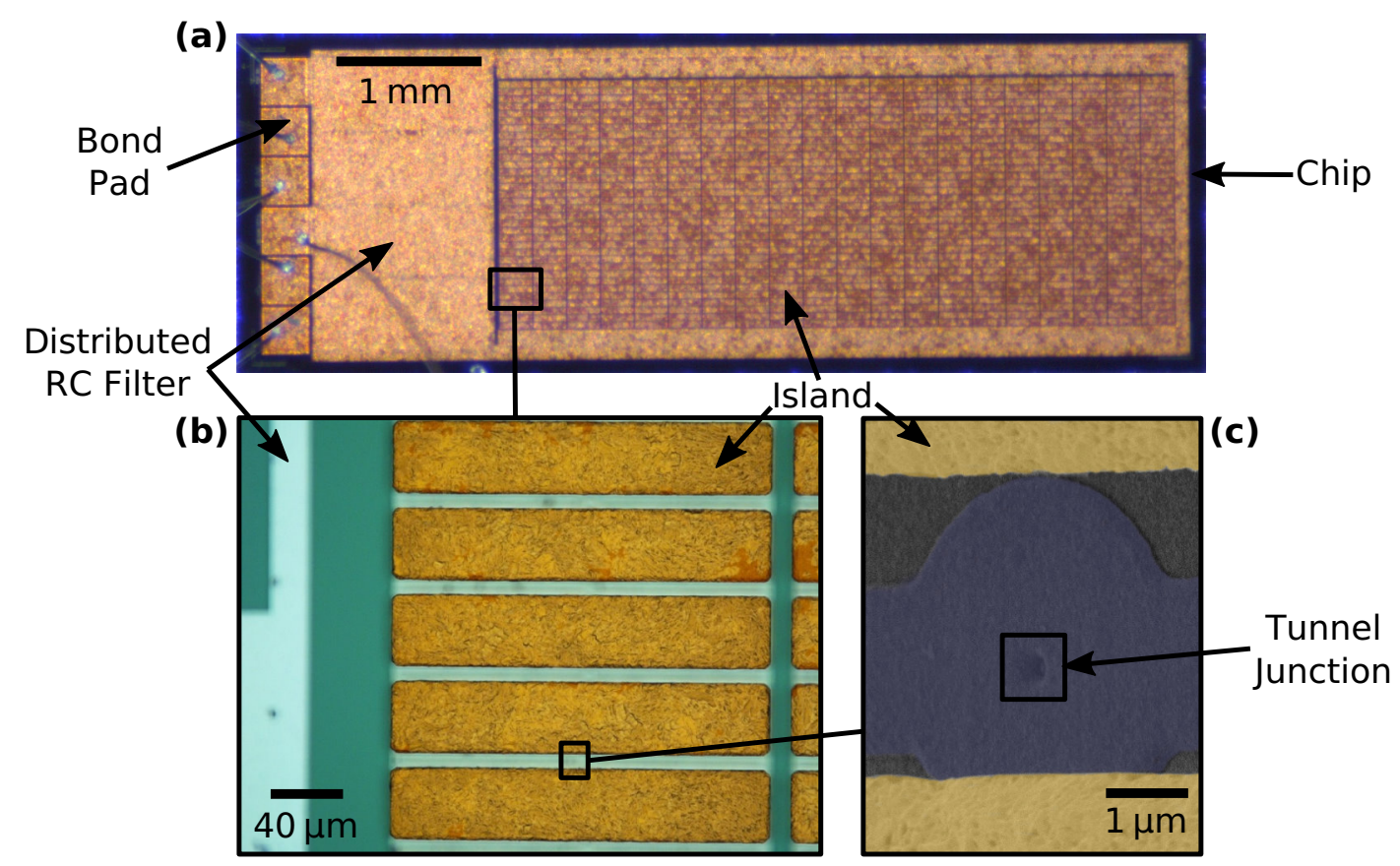

Figure 3.1: Coulomb Blockade Thermometer Chip. Panel (a) shows the $6.5 \mathrm{~mm} \times 2.3 \mathrm{~mm}$ chip with the large island array on the right taking up most of the chip, the distributed RC (Resistor-Capacitor) filter (covered with a ground plane so not visible) on the left and six bond pads at the leftmost edge. From top to bottom, the first and fourth bond pads are ground, the second and third are connected to one side of the array, and the fifth and sixth connect to the other side. Panel (b) is optical micrograph of five of the islands. Panel (c) is a false-coloured scanning electron microscope (SEM) micrograph taken between two of the islands showing the cylindrical via containing the tunnel junction. The images in panels (a) and (b) were used in a redrawn form in [88] and the image in panel (c) was used without false colour in [49].

and thickness $675 \mu \mathrm{m}$ with a $300 \mathrm{~nm}$ thick insulating thermal oxide $\left(\mathrm{SiO}_{\mathrm{x}}\right)$ layer on the surface. The device has a $20 \times 33$ array of tunnel junctions, creating 20 parallel rows, each with 32 metallic islands, as shown in Figure 3.1a. The electronic structure is fabricated in four distinct steps, leading to a structure containing four vertically stacked layers. These are the copper cooling fins (Figure 3.1b), which are above the top electrode which is separated from the bottom electrode by an insulating layer through which the tunnel junction passes in a via, see Figures $3.1 \mathrm{c}$ and 3.2 .

The electrode layers consist of a $250 \mathrm{~nm}$ thick aluminium film, deposited using DC magnetron sputtering and subsequently patterned by etching through a resist exposed using UV lithography. The etching was performed using a $\mathrm{Cl}_{2}$ and $\mathrm{BCl}_{3}$ plasma. The insulating layer was created by covering the lower electrode with $250 \mathrm{~nm}$ thick $\mathrm{SiO}_{2}$ deposited using Plasma-Enhanced Chemical Vapour De- 


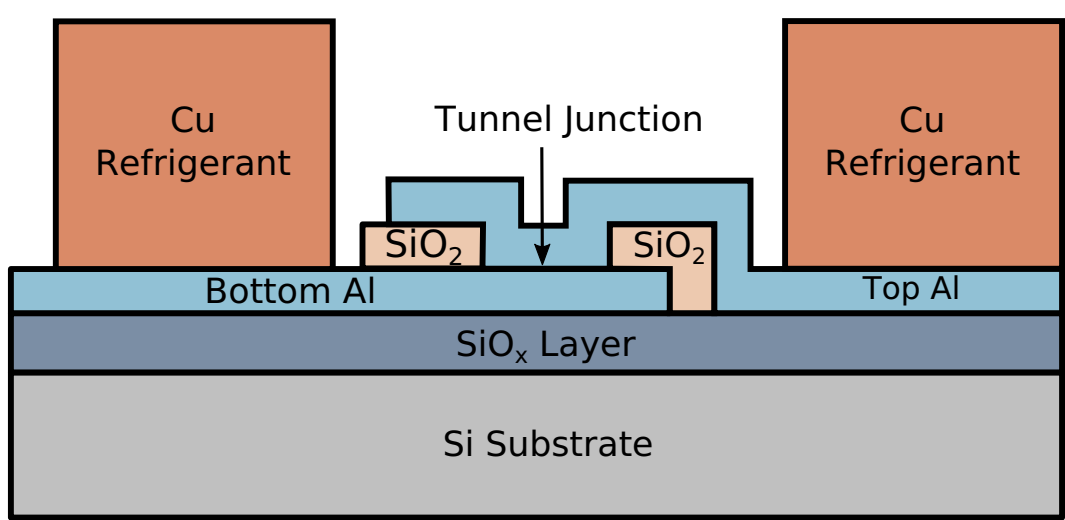

Figure 3.2: Coulomb Blockade Thermometer Junction Structure. The tunnel junction is defined by a hole in the $\mathrm{SiO}_{2}$ insulating layer and is formed between the two layers of aluminium by controlled oxidation of the lower layer before depositing the upper.

position (PECVD). The holes, or vias, for the tunnel junctions to pass through are $800 \mathrm{~nm}$ in diameter and were created by etching the $\mathrm{SiO}_{2}$ using a $\mathrm{CF}_{4}$ and $\mathrm{CHF}_{3}$ plasma. Before the deposition of the top electrode, the native oxide layer on the aluminium (which forms while the sample is exposed to air) was removed using an Ar plasma and then a controlled oxide layer was created in 110 mbar of oxygen over $6000 \mathrm{~s}$ while the sample was held at $180^{\circ} \mathrm{C}$. The top electrode layer was then deposited without breaking the vacuum. The resulting junctions have a resistivity of $12 \mathrm{k} \Omega \mu \mathrm{m}^{2}$ [89]. Aluminium is a superconductor with critical temperature $T_{c}=1.19 \mathrm{~K}$ [37] and a critical field of $B_{c}=10 \mathrm{mT}$ in the bulk at zero temperature [90], which is greater for thin films [91]. Since a CBT is designed to operate with normal metal islands, a magnetic field of at least $100 \mathrm{mT}$ must be applied for the device to function properly.

This technique for fabricating $\mathrm{AlO}_{\mathrm{x}}$ tunnel junctions differs from the more common shadow angle deposition technique [84] in that the vacuum is broken between the two aluminium deposition stages, and is hence termed an ex-situ process (in contrast with in-situ angle deposition). This allows greater flexibility in the patterning process for the device, prevents the creation of unwanted shadow structures, and leads to a small deviation (average 1.3\%) in tunnel junction resistance between different junctions in the same device, allowing the creation of more uniform arrays [92]. This gives an alternative to using single junction thermometry as a solution to the inaccuracies that result from non-uniform junction arrays [93].

The on-chip refrigerant is applied on top of the aluminium islands by electroplating copper through a mask, creating blocks $6.14 \mu \mathrm{m}$ thick on each island of crosssection $39 \mu \mathrm{m} \times 206 \mu \mathrm{m}$ (Figure 3.1b). If, instead of electroplating, the blocks were applied using the more conventional nanofabrication techniques of evaporation 
or sputtering, the thickness of the film means that the copper would have builtin stress. This stress is a common cause of thin-film failure [94], particularly at low temperatures where the differential contraction on cooling can then lead to mechanical breakage. In addition, the stress leads to poor thermal contact at the interface, limiting the degree to which the aluminium can be cooled by the refrigerant blocks [49]. The chemical environment during the electroplating process removes the native oxide from the aluminium, and hence ensures that the copper is in direct electrical connection with the aluminium islands, allowing electron thermalisation across the boundary without relying on electron-phonon coupling to transport the heat through the oxide layer.

The island array is connected to two leads per side to enable four-terminal measurement of the device conductance and the application of DC bias. Each of these four leads features an integrated on-chip filter to reduce noise which would otherwise complicate the measurement of the CBT and cause elevated electron temperatures [95]. This filter consists of a long meander fabricated on top of a ground plane (see Figures 3.1a and 3.1b), hence providing a distributed capacitance of $\approx 10 \mathrm{pF}$ with a distributed resistance of $\approx 500 \Omega$. The long meander also assists with thermalising the electrons before they reach the first tunnel junction, helping to achieve a uniform electron temperature across the array. After the filter, the four leads connect to four bond pads (Figure 3.1a) to enable connection to the external circuitry. There are two additional bond pads to provide an earth connection for the chip ground plane.

\subsection{Experimental Set-Up}

This section describes the preparation required to enable measurement of the CBT in a dilution refrigerator. The methods used to mount the CBT in the dilution refrigerator, with particular attention paid to the techniques used to ensure the precooling is effective, are detailed in Subsection 3.2.1. The electrical techniques used to permit measurement of the CBT with the minimum of noise are then explained in Subsection 3.2.2.

\subsubsection{Mounting and Heatsinking}

The CBT device is mounted in a silver package (Figures 3.3a and 3.3b) using Electrodag® silver paste to provide a high thermal conductivity connection between the package and the silicon substrate. The package is designed to maximise thermal conductance between its exterior surfaces and the CBT, while minimising the cross sectional area perpendicular to the direction of the applied magnetic field. This 


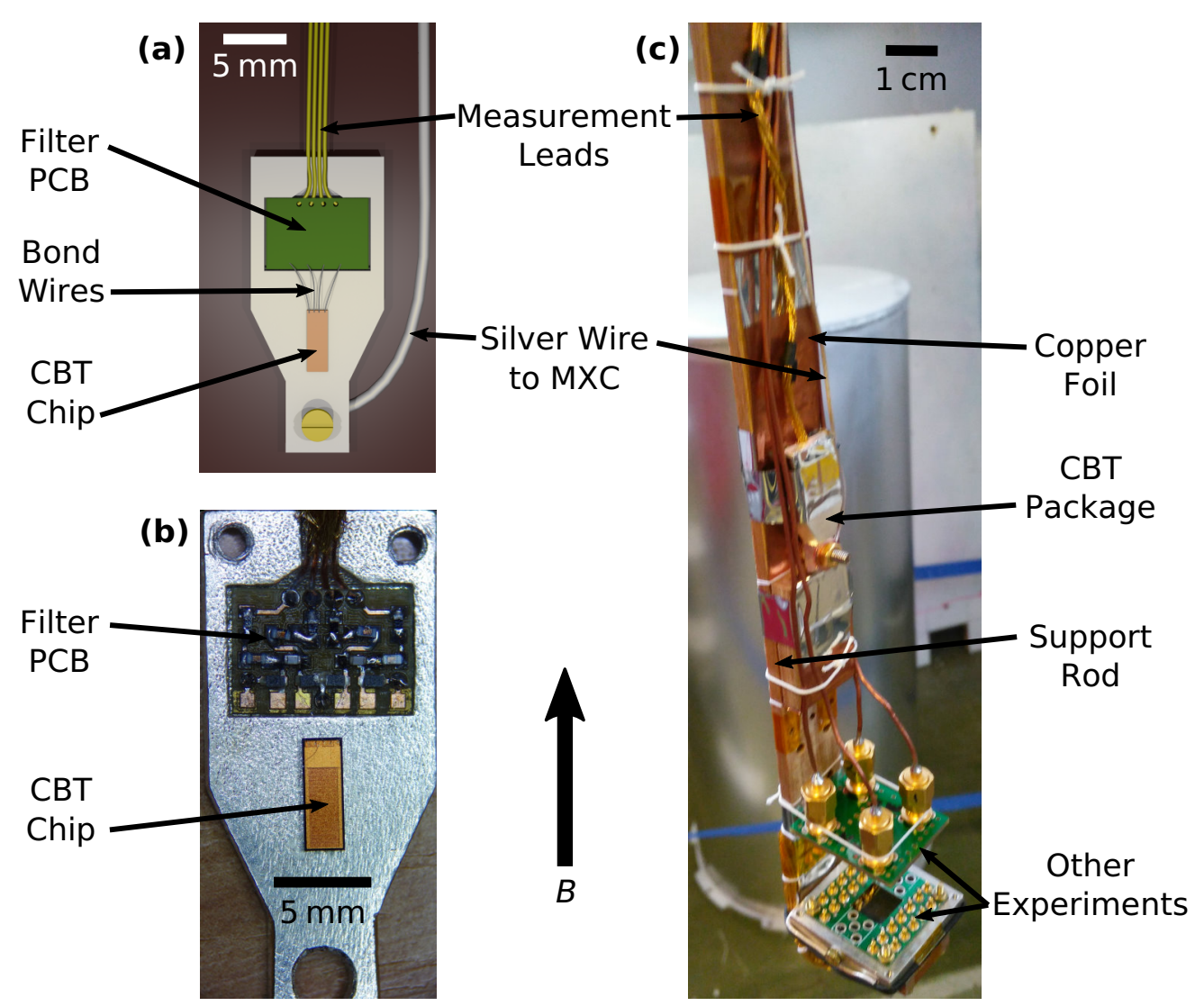

Figure 3.3: CBT Mounting in the Dry Cryostat. Panel (a) is a schematic rendering of the opened CBT package and is redrawn from [88]. Panel (b) is a photograph of the opened package showing the CBT in situ. Panel (c) is a photograph of the 'coldfinger' attached below the MXC plate of the dilution refrigerator. This passes into the bore of the $5 \mathrm{~T}$ magnet allowing the $\mathrm{CBT}$ and other experiments to be located within its field. The magnetic field $B$ is applied in the direction shown.

is to ensure that the heat flow to the MXC plate of the dilution refrigerator is maximised during pre-cooling, and that eddy current heating is as small as possible while the magnetic field is swept during demagnetisation. The package was manufactured by casting raw silver into a 3D-printed mould and has two parts: a base to which the CBT is mounted and a lid which tightly fits over the base. The closed package therefore provides mechanical and electrostatic discharge (ESD) protection while also acting as a noise shield.

The package features an M3 clearance hole at the bottom which is surrounded by a flat plate on both the base and lid. A high purity, annealed silver wire of $\approx 1 \mathrm{~mm}$ diameter and $\approx 30 \mathrm{~cm}$ length is attached to the package using this hole and to the gold-plated copper MXC plate of the dilution refrigerator to provide the thermal link for pre-cooling (Figures 3.3b and 3.3c). At each end of the wire an annulus is formed by squashing a short loop of silver wire in a press. The connection to the silver package is then made using a brass bolt through the annulus and package 
which is then secured using a brass nut on top of a copper washer. The copper washer is required because of the difference in the thermal expansion coefficients between silver $\left(\alpha=19.4 \times 10^{-6} \mathrm{~K}^{-1}\right)$ and brass $\left(\alpha=18.5 \times 10^{-6} \mathrm{~K}^{-1}\right)$ meaning that, on cooling, the nut and bolt will become loose as the silver package shrinks more than the bolt. Adding a copper $\left(\alpha=16.4 \times 10^{-6} \mathrm{~K}^{-1}\right)$ or molybdenum $\left(\alpha=5.0 \times 10^{-6} \mathrm{~K}^{-1}\right)$ washer of sufficient thickness allows the resulting slack to be taken up as these shrink less than brass. The connection to the MXC plate is similar, however no copper washers are required as the plate itself is made from copper.

A Bluefors LD250 dilution refrigerator was used to pre-cool the CBT and the surrounding environment. This is a commercial dilution refrigerator with the first two cooling stages being provided by a pulse tube cryocooler, hence this is known as a 'dry' dilution refrigerator, c.f. a wet dilution refrigerator which uses a liquid helium bath for the first cooling stage, often with a liquid nitrogen outer shielding stage. This machine has a base temperature of $7 \mathrm{mK}$ with a nominal cooling power of $250 \mu \mathrm{W}$ at $100 \mathrm{mK}$. The package itself is mounted approximately $25 \mathrm{~cm}$ below the MXC plate such that it is within the bore of a $5 \mathrm{~T}$ superconducting solenoid magnet. It is fixed in place to a Tufnol® plastic rod laminated with copper foil which provides some further heat sinking but negligible cross section for eddy current heating (Figure 3.3c).

Above the CBT, in the package, a printed circuit board (PCB) is mounted. This PCB holds the bond pads which the device is connected to by ultrasonically bonding $25 \mu \mathrm{m}$ aluminium wire between these pads on the PCB and the corresponding ones on the CBT sensor. The PCB also has an RLC (Resistor-Inductor-Capacitor) filter, made from discrete components and described in Subsection 3.2.2.

A further filter is placed at, and thermally anchored to, the MXC plate. This is an RCR (Resistor-Capacitor-Resistor) pi-filter made from discrete components. It is a commercial product (Aivon Therma) which is designed to aid thermalisation of the measurement lines at the base temperature of the dilution refrigerator. The filter used here is modified from the original Aivon design by coating (potting) the filter PCB with Stycast ${ }^{\circledR}$ 2850GT epoxy loaded with copper powder. This modification ensures that the resistors within the filter remain cool, as they are otherwise only in contact with a vacuum, a superconductor (the solder between the components and PCB) and the fibreglass board (FR4 PCB material), all of which have very poor thermal conductivity. The Stycast ${ }^{\circledR}$ epoxy however is designed as a high thermal conductivity potting compound for circuit boards, and is known to retain a relatively large thermal conductivity at millikelvin temperatures [96, 97]. 


\subsubsection{Electrical}

A four-terminal, or Kelvin, resistance measurement is performed on the CBT to null out the additional resistance present in the measurement leads in the cryostat and from the resistors in the three filters used between the measurement equipment and CBT array. The measurement circuit is shown schematically in Figure 3.4 and operates by driving a combined alternating current (AC) excitation and direct current (DC) bias current and then measuring the AC and DC voltages resulting from this using a lock-in amplifier and voltmeter, respectively.

The conductance at any particular DC bias is measured by observing the AC voltage created by the $\mathrm{AC}$ excitation current, which in this study was at a frequency of $83 \mathrm{~Hz}$. The output of the function generator $V_{I a c}$ is used to set the output of a voltage-controlled current source:

$$
I_{\mathrm{ac}}=V_{\mathrm{Iac}} \times A_{\mathrm{ac}},
$$

for $A_{a c}$ the AC gain of the current source. This AC current then causes a voltage

$$
V_{\mathrm{ac}}=I_{\mathrm{ac}} \times \mathrm{R}_{\mathrm{CBT}}\left(\mathrm{V}_{\mathrm{dc}}, \mathrm{T}_{\mathrm{e}}\right)=V_{\mathrm{Iac}} A_{\mathrm{ac}} \mathrm{R}_{\mathrm{CBT}}\left(\mathrm{V}_{\mathrm{dc}}, \mathrm{T}_{\mathrm{e}}\right)
$$

across the CBT, where $R_{\mathrm{CBT}}\left(\mathrm{V}_{\mathrm{dc}}, \mathrm{T}_{\mathrm{e}}\right)$ is the resistance of the CBT which is a function of the DC bias voltage $V_{\mathrm{dc}}$ and electron temperature $T_{e}$, as described in Section 2.2. This signal is multiplied by the gain of a voltage preamplifier $A_{V}$ and then is measured by the lock-in amplifier. The preamplifier used here is a Stanford Research Systems SR560, a low-noise, optionally battery powered, voltage preamplifier with selectable input filters. The SR560 was used with a gain of 1000 and a low pass filter with cutoff frequency $10 \mathrm{kHz}$ and roll-off $12 \mathrm{~dB} /$ octave. Although shown separately in the schematic, the function generator and lock-in amplifier were one unit with an internal reference link, a Signal Recovery 7265. The DC bias across the sensor $V_{\mathrm{dc}}$ is set indirectly via the current

$$
I_{\mathrm{dc}}=V_{\mathrm{Idc}} \times A_{\mathrm{dc}}
$$

for $A_{\mathrm{dc}}$ the DC gain of the current source and $V_{\mathrm{Idc}}$ the output of the DC voltage source, a Stanford Research Systems SIM928 isolated voltage source ${ }^{1}$. This then

\footnotetext{
${ }^{1}$ These voltage sources consist of a pair of nominal $27 \mathrm{~V}$ batteries which are interchangeably connected via a controllable linear regulator to the output. This gives an almost completely ripple free output with no connection to mains earth, avoiding ground loops and minimising noise in the measurement circuit.
} 


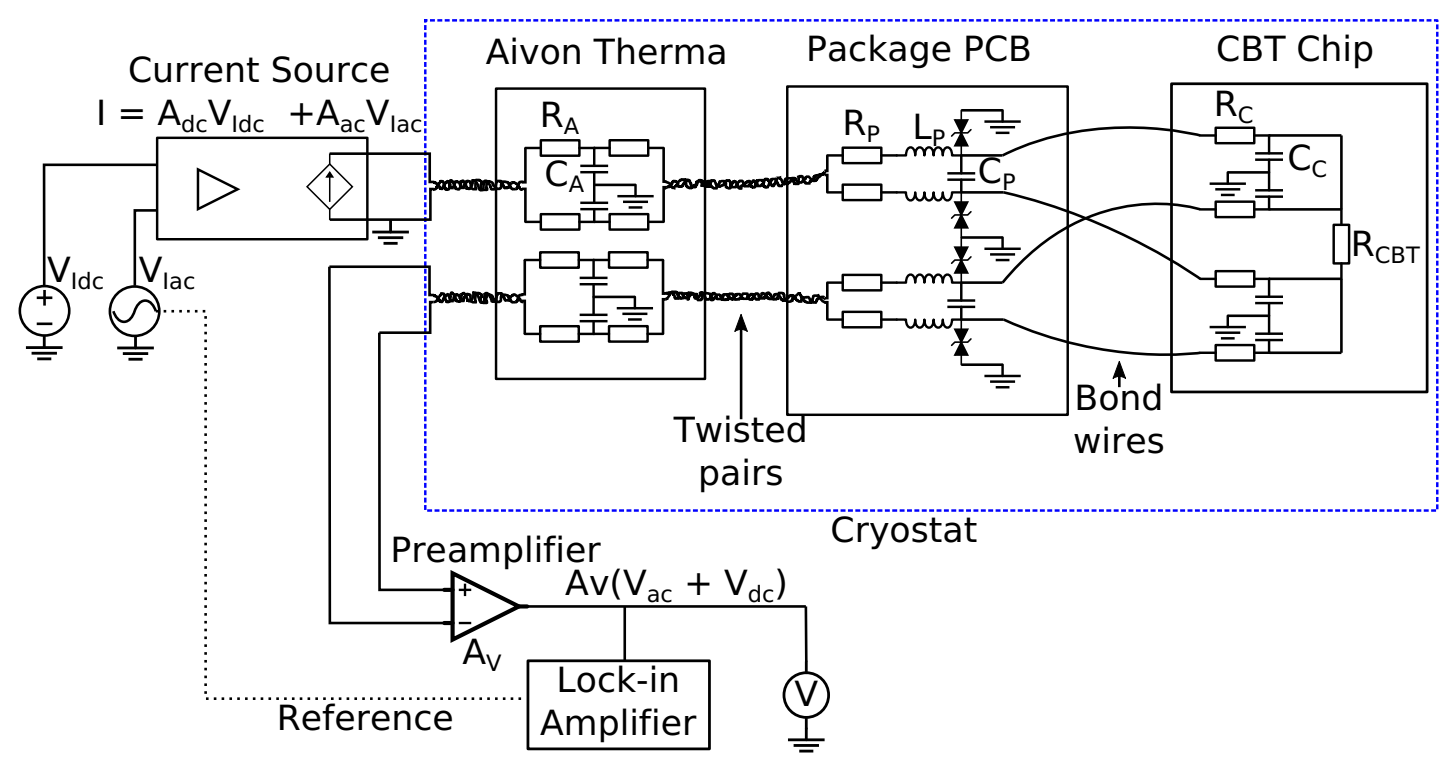

Figure 3.4: CBT Measurement Electrical Schematic. The voltage controlled current source outputs $A_{\mathrm{ac}}=10 \mathrm{nA} \mathrm{V}^{-1}$ and $A_{\mathrm{dc}}=1 \mathrm{\mu A} \mathrm{V}^{-1}$ in response to the $\mathrm{AC}$ and $\mathrm{DC}$ input voltages, respectively. In each of the three filters, all the components of the same type have the same nominal value. In the Aivon Therma filter these values are $R_{A}=200 \Omega$ and $C_{A}=1 \mathrm{nF}$. On the package PCB they are $R_{P}=5 \mathrm{k} \Omega, L_{P}=24 \mathrm{nH}$ and $C_{P}=180 \mathrm{pF}$. The distributed filter on the CBT chip has $R_{C} \approx 500 \Omega$ and $C_{C} \approx 10 \mathrm{pF}$. The temperature and bias dependent resistance of the CBT island array is represented by $R_{\mathrm{CBT}}$.

creates the voltage bias

$$
V_{\mathrm{dc}}=I_{\mathrm{dc}} \times \mathrm{R}_{\mathrm{CBT}}\left(\mathrm{V}_{\mathrm{dc}}, \mathrm{T}_{\mathrm{e}}\right)=V_{\mathrm{Idc}} A_{\mathrm{dc}} \mathrm{R}_{\mathrm{CBT}}\left(\mathrm{V}_{\mathrm{dc}}, \mathrm{T}_{\mathrm{e}}\right)
$$

which is verified by measuring it, after the voltage gain $A_{V}$ is applied by the preamplifier, with the DC voltmeter. The current source was custom made by the electronics workshop in Lancaster, while the lock-in amplifier, voltage source and voltmeter are all under computer control to allow automated measurements of the conductance curve.

The circuit has several features designed to minimise the electrical noise within the circuit itself, and especially the noise across the sensor, because reaching and measuring the lowest electron temperatures in a CBT requires a low noise environment [49, 95] for two main reasons. Firstly, electrical noise of a sufficient amplitude causes parasitic heating of the sensor by Joule heating. When combined with the limited heat capacities, and hence cooling powers, available during an on-chip demagnetisation experiment, this inherently limits the attainable base temperatures. Secondly, voltage or current noise across the tunnel junctions can lead to the smearing out of the conductance curve, causing an inaccurately high 


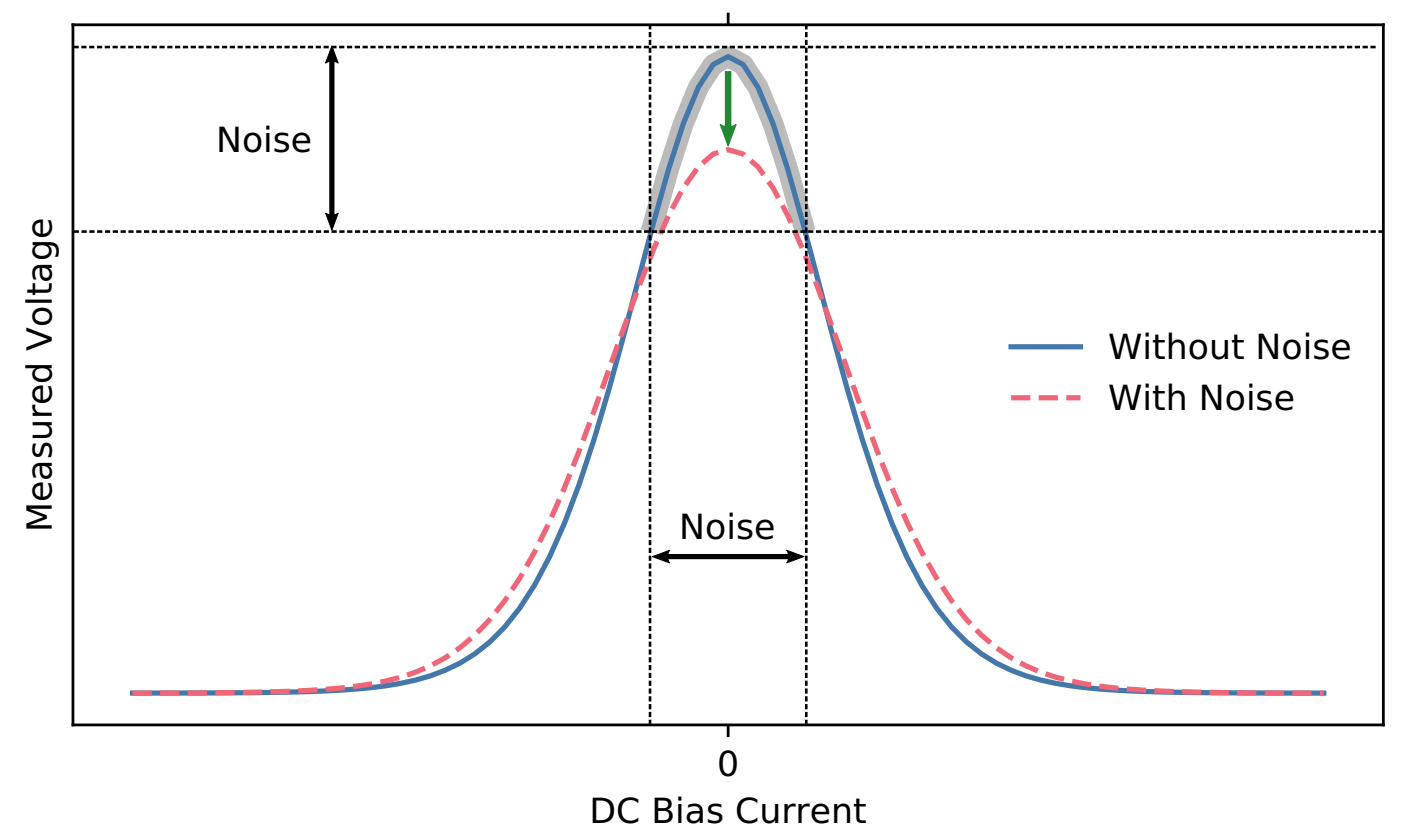

Figure 3.5: CBT Conductance Curve Broadening in Response to Noise. The true I-V curve is shown by the solid blue line. If current noise applied to the CBT (exaggerated here), the $I-V$ curve is repeatedly scanned in the vicinity of the desired DC bias, as shown by the grey shaded section for zero bias. This scanning leads to changes in the amplitude of the excitation signal, which is averaged by the lock-in over its time constant. This averaging produces a moving average of the $I-V$ curve, as shown by the dashed red line. At zero bias, this causes a peak voltage smaller than the true maximum (green arrow), which gets interpreted during analysis as a higher electron temperature.

electron temperature to be measured. This occurs as large noise signals mean that the lock-in amplifier sees measurements over a broad area of the conductance dip, resulting in a smaller average voltage, as shown in Figure 3.5. This effect also occurs if an excessive excitation is used to measure the conductance, hence it must be kept small (40 pA for these measurements). This small excitation means in turn that measurements will be difficult if the noise is not reduced such that an acceptable SNR is reached.

The measurement leads are two sets of twisted pairs, chosen to limit the loop area in the circuit otherwise available for the inductive pickup of electrical noise, and are arranged to create a balanced line by having the positive and negative sides of the circuit within each pair and such that both of the pairs are inside the same cable. To this end, the pairs are denoted $I_{ \pm}$on the current drive side of the four-terminal measurement and $V_{ \pm}$on the voltage sense side. The twisted pairs are made from 35 AWG (american wire gauge, equivalent to $0.016 \mathrm{~mm}^{2}$ ) $\mathrm{PhBr}$ (Phosphor-Bronze alloy) wire which has an end-to-end resistance of $20 \Omega$ when the 
dilution refrigerator is at base temperature, and a capacitance between the wires in a pair of ${ }^{2} \sim 1 \mathrm{nF}$. This creates a $\sim 10 \mathrm{MHz}$ distributed low-pass filter in a similar manner to Thermocoax ${ }^{\circledR}$ coaxial cable [98]. Although in principle a balanced line rejects noise most effectively if both sides have an equal impedance to earth [99], in practice it is difficult to ensure this condition is met due to stray capacitances, hence it was found that connecting the negative output of the current source to ground reduced the noise in the circuit.

All four of the measurement leads pass through three low temperature, low pass filters between the CBT island array and the room temperature electronics. The Aivon Therma filter is a symmetric RCR filter with a cutoff frequency (half power point) of $800 \mathrm{kHz}$, made from discrete surface-mount resistors and capacitors. It removes common and differential mode noise incoming from outside the dilution refrigerator and outgoing noise picked up by the twisted pairs between the filter and CBT package. As stated in Subsection 3.2.1, this filter was modified from its original form by potting it in epoxy to aid thermalisation. Additionally, however, copper powder ('325 mesh') was mixed with the epoxy to a concentration of $40 \%$ by mass to create a rudimentary microwave filter, since above $\sim 1 \mathrm{GHz}$ the attenuation from a filter made from discrete components is small [100]. This operates by eddy current dissipation in the metal particles enhanced by the skin effect at high frequencies $[61,65,101]$.

The second filter, built on a PCB within the CBT package and located between the incoming measurement leads and the bond pads, is a RLC low-pass filter for radio frequency $(\mathrm{RF})$ differential noise and has a cutoff frequency of $80 \mathrm{MHz}$. It is designed to complete the RF shielding created by the CBT package by rejecting incoming RF on the measurement leads. This board additionally has ESD protection diodes between each of the four bond pads and ground to protect the sensor from large transient voltages on the measurement lines. The final filter is the distributed, on-chip, RC low pass filter which has a cutoff frequency of $30 \mathrm{MHz}$.

\subsection{CBT Characteristics}

With the CBT installed in the dilution refrigerator and cooled to millikelvin temperatures, full conductance curves against bias voltage were recorded at four different temperatures, as shown in Figure 3.6. This was performed by heating the MXC plate of the dilution refrigerator using a resistive heater, during which the temperature of this plate could be monitored using a $\mathrm{RuO}_{2}$ thermometer, supplied

\footnotetext{
${ }^{2}$ This capacitance was only roughly measured using the capacitance function on a multimeter which only had $1 \mathrm{nF}$ resolution.
} 


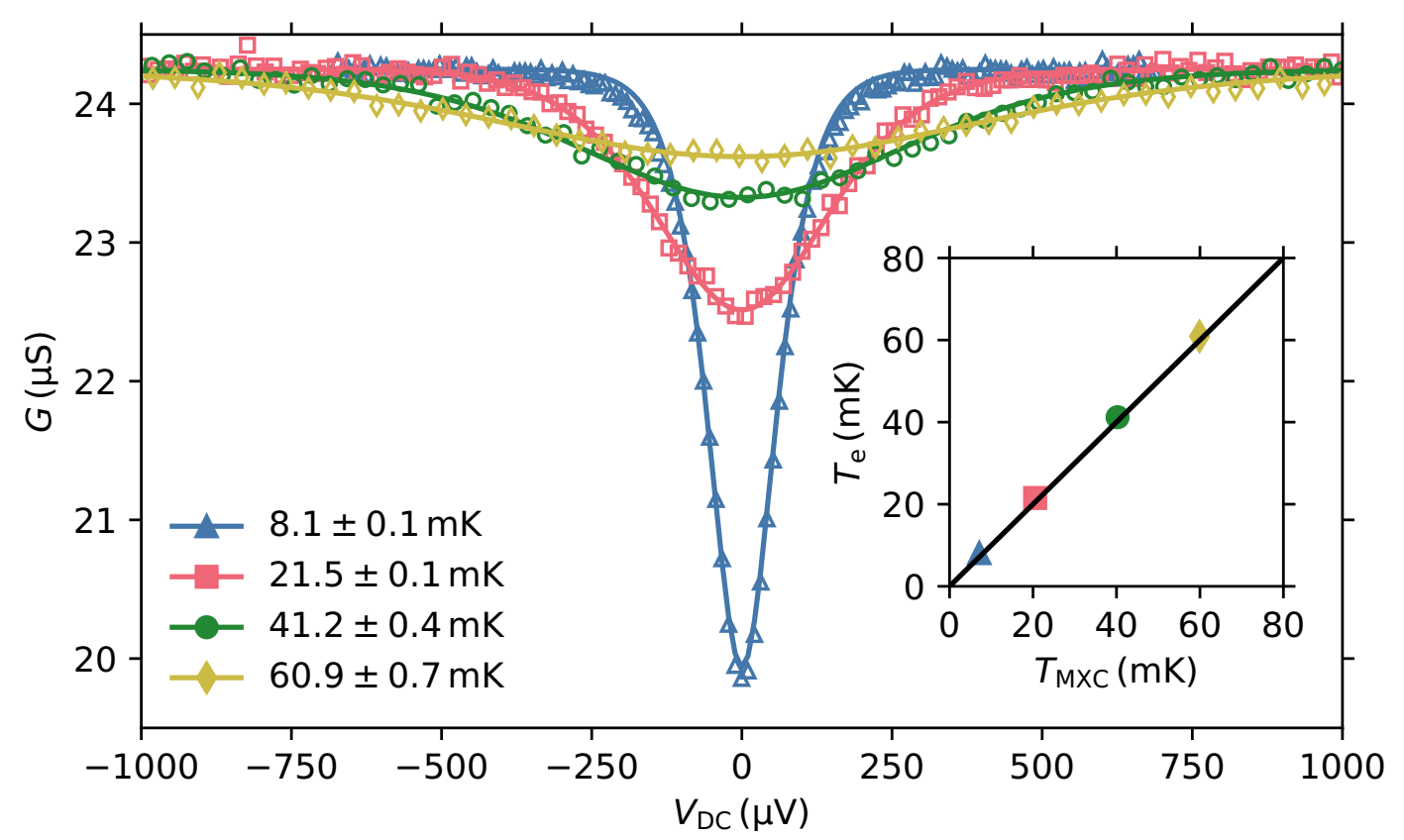

Figure 3.6: CBT Conductance Curves at 0.1 T. The symbols show the measured values and the lines show the fits to a full tunnelling model, from which the temperatures shown in the legend are extracted. The inset shows the extracted electron temperature against the temperature the mixing chamber was heated to. The solid line in the inset is $T_{e}=T_{\mathrm{MXC}}$ for reference. This figure is redrawn from [88].

and calibrated by Bluefors Cryogenics. Once a stable mixing chamber temperature was reached, a conductance curve was recorded and then the process repeated for a total of three temperatures. Finally, the MXC and CBT were allowed to return to their base temperature and a final conductance curve recorded. For all of these measurements, the applied magnetic field was set to the minimum used throughout this study of $0.1 \mathrm{~T}$, used to ensure that the aluminium in the CBT does not superconduct.

The three warmest curves in Figure 3.6 were simultaneously fitted, using code based on the free, open source python library pyCBT [1], to a full tunnelling model by allowing the sensors parameters $R_{T}$ and $C_{\Sigma}$ in addition to the electron temperatures to vary. In the fitting, the sensor parameters are common to all three fitted curves, while the electron temperatures were allowed to differ. The coldest curve was then fitted by varying the electron temperature only and using the sensor parameters found in the previous fitting, allowing the validity of these parameters to be verified. The electron temperatures $\left(T_{e}\right)$ extracted from the fitting are plotted against the MXC temperature $\left(T_{\mathrm{MXC}}\right)$ measured by the $\mathrm{RuO}_{2}$ thermometer in the inset. The line $T_{e}=T_{\mathrm{MXC}}$ is also plotted in the inset to show the degree of thermalisation of the electrons with the MXC plate. 


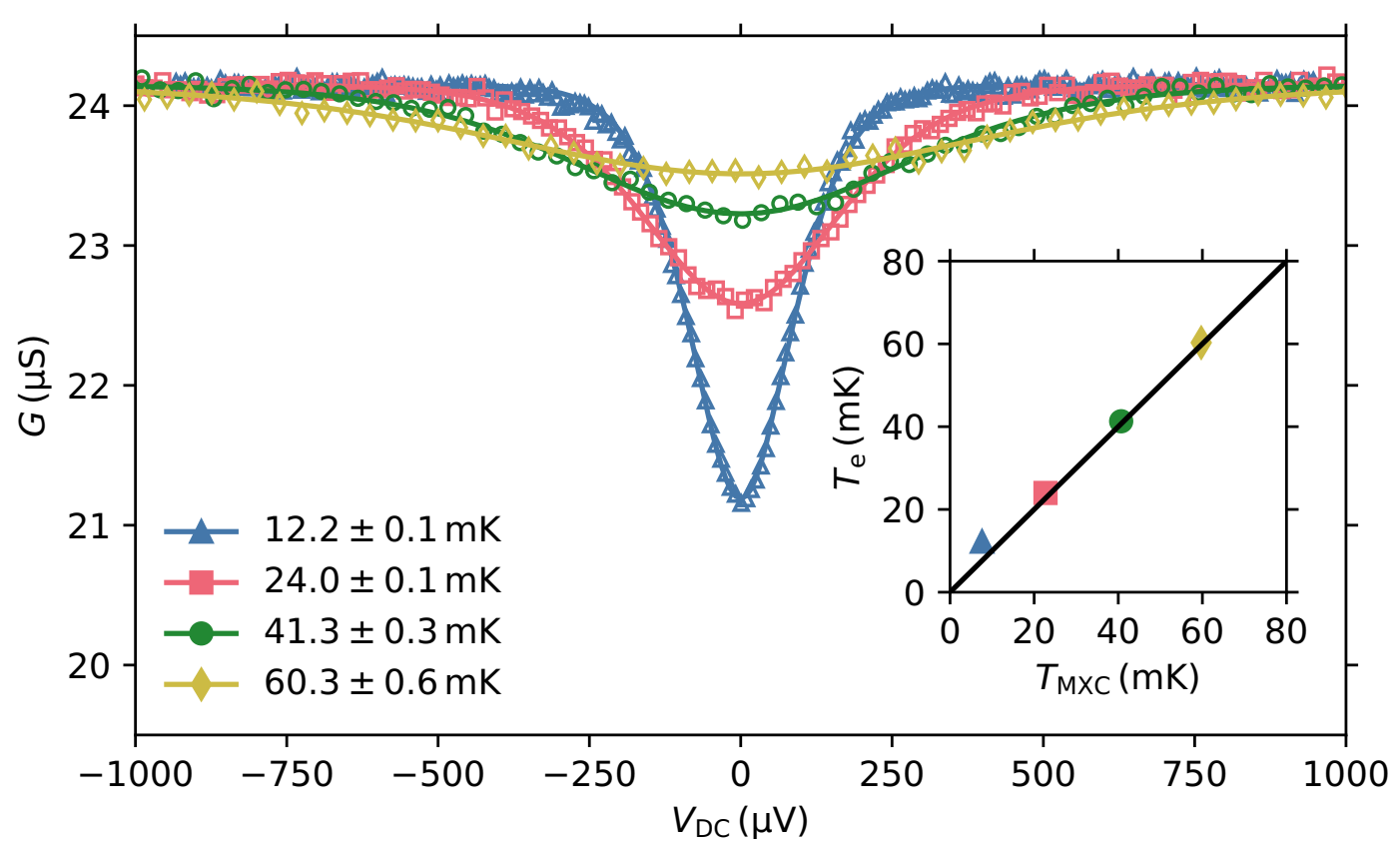

Figure 3.7: CBT Conductance Curves at 5.0 T. This figure is plotted in the same manner as Figure 3.6 and is redrawn from [88].

Four more conductance curves were measured at a magnetic field of $5.0 \mathrm{~T}$, as shown in Figure 3.7. This allows the sensor parameters to be compared between the two fields so as to verify, as has been done for other CBTs [47, 102], that the operation of this particular CBT is not sensitive to magnetic field (other than the requirement to ensure the suppression of superconductivity), as would be expected given that they are entirely electrostatic devices [23]. In Table 3.1 we see that the values of $C_{\Sigma}$ are consistent within 1 standard deviation $(1 \sigma)$, as expected. The values of $R_{T}$ are in agreement within $1.4 \sigma$ which is more ambiguous, although they are still close. The slight difference between the two may have arisen due to the drift in gain of the preamplifier with time or room temperature variation, since $R_{T}$ is determined directly from the high bias conductance which changes linearly with amplifier gain. Another possibility is that there is a slight magnetoresistive effect, as has been seen in another study [103] where $R_{T}$ varied by $7 \%$ up to $3 \mathrm{~T}$, however the variation seen here is only $0.4 \%$, which is consistent with another study which concluded that the magnetic field has no effect after observing CBT readings within $1 \%$ for fields up to $23 \mathrm{~T}$ [104].

The averages of the sensor parameters, weighted by the inverse of the uncertainty squared, are $C_{\Sigma}=(192.1 \pm 0.6) \mathrm{fF}$ and $R_{T}=(25.05 \pm 0.04) \mathrm{k} \Omega$. To perform the self-calibration procedure, used to relate the zero bias conductance to the electron temperature for this particular sensor (see Subsection 2.2.3), the full tunnelling model was used to calculate a look-up table of conductances for a range of tem- 


\begin{tabular}{ccc}
\hline Field & \multicolumn{2}{c}{ CBT Parameter } \\
\cline { 2 - 3 }$B(\mathrm{~T})$ & $C_{\Sigma}(\mathrm{fF})$ & $R_{T}(\mathrm{k} \Omega)$ \\
\hline 0.1 & $192.4 \pm 0.9$ & $24.99 \pm 0.06$ \\
5.0 & $191.9 \pm 0.8$ & $25.10 \pm 0.06$ \\
\hline
\end{tabular}

Table 3.1: CBT Sensor Parameters. These parameters were extracted from the fit to the full tunnelling model, as shown in Figures 3.6 and 3.7.

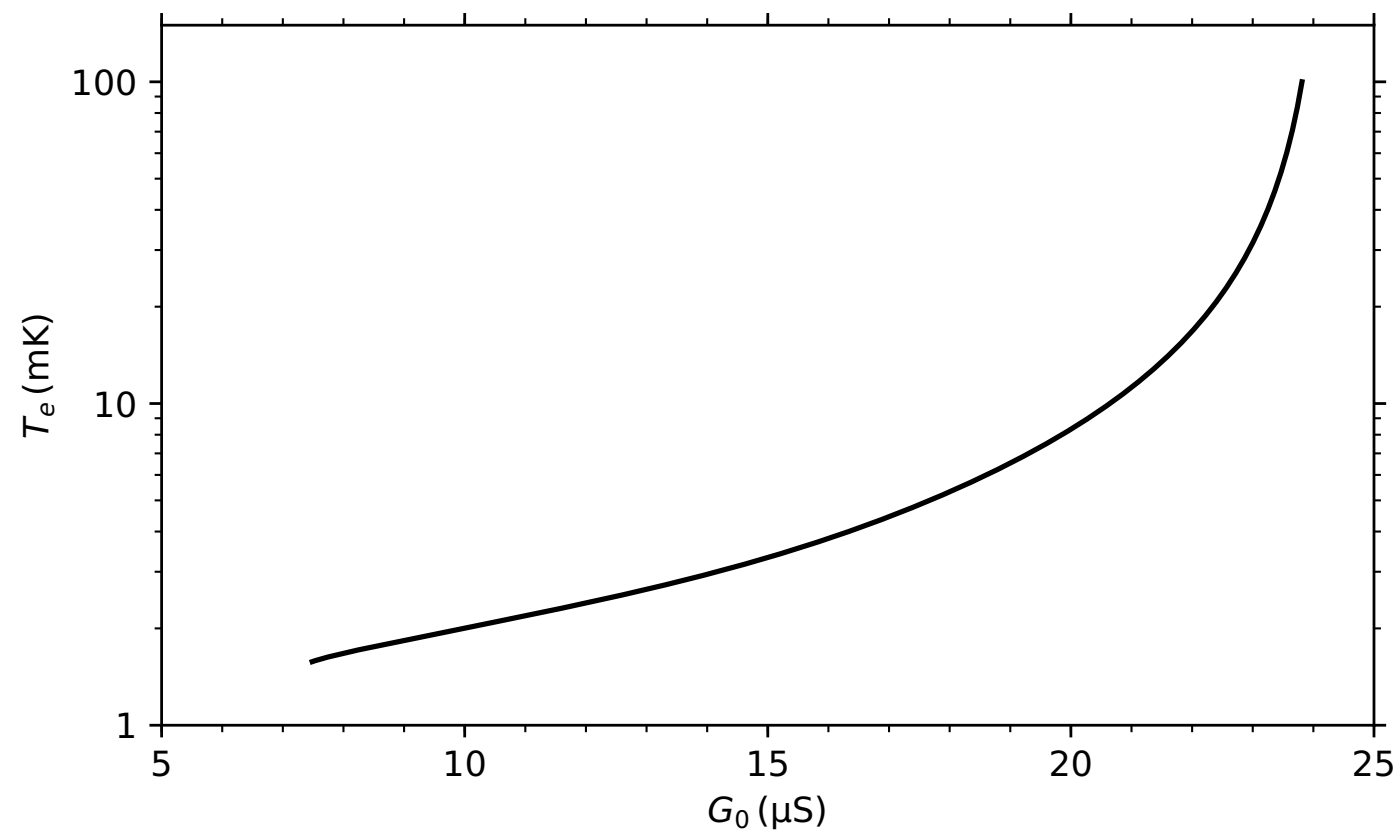

Figure 3.8: CBT Temperature vs. Conductance Self-Calibration Curve. The data in this plot is used to calculate the CBT's electron temperature $T_{e}$ from a measurement of the zero bias conductance $G_{0}$.

peratures. The electron temperature can then be found from a set of zero bias conductance data by interpolating between the (tightly spaced) reference points in the lookup table. This table is plotted in graphical form in Figure 3.8.

The exact temperatures that the MXC plate was heated to during these measurements are unimportant and are not required for the fitting, nor for the selfcalibration procedure. It is, however, useful to compare the measured electron temperatures with the set MXC temperatures to verify the quality of the fit and investigate the heatsinking of the CBT. The two temperatures are compared in the insets of Figures 3.6 and 3.7, and the degree of overheating $\left(T_{e}-T_{\mathrm{MXC}}\right)$ is shown in Figure 3.9. Here we see that at $0.1 \mathrm{~T}$ there is a constant overheating of $\approx 1 \mathrm{mK}$ for all four data points, while at $5.0 \mathrm{~T}$ there is greater overheating at lower temperatures. This occurs because at full magnetic field small vibrations result in noticeable eddy current heating [64]. The overheating is greater at lower 


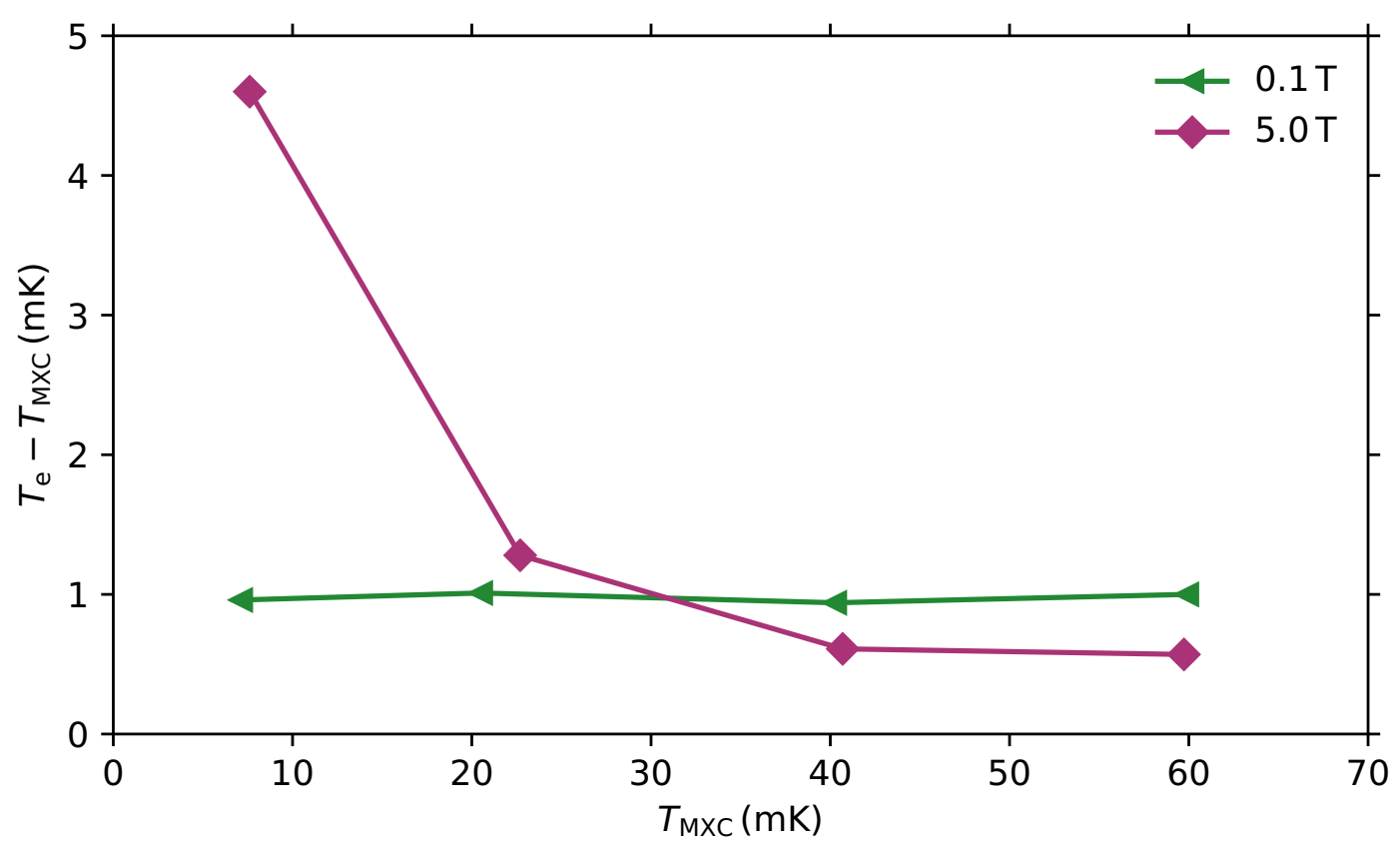

Figure 3.9: Thermalisation of the CBT. For each of the temperatures the MXC plate was set to, $T_{\mathrm{MXC}}$, the amount of overheating of the CBT's electrons is shown at both $0.1 \mathrm{~T}$ and $5.0 \mathrm{~T}$.

temperatures since the electron-phonon coupling is weaker (Equation 2.45). At higher temperatures, there is apparently a greater amount of overheating at $0.1 \mathrm{~T}$ than at $5.0 \mathrm{~T}$, however this could be due to the slight magnetoresistance of the $\mathrm{RuO}_{2}$ thermometer used to measure the MXC plate temperature [105].

\subsection{CBT Cooling}

In this section, the results from attempting on-chip cooling on the CBT are described. The initial observation and verification of cooling are presented in Subsection 3.4.1. Secondly, a thermal model for the cooling is shown in Subsection 3.4.2 and then this is used to optimise the process in Subsection 3.4.3.

\subsubsection{Initial Experiments}

For demagnetisation, the magnetic field is first increased to its maximum of 5.0 T. During this, the CBT electrons and the dilution refrigerator itself are warmed by the heat of magnetisation and eddy current heating. The solenoid is then held at full field using the magnet power supply until the dilution refrigerator and the CBT electrons are thermalised, a process that takes approximately $14 \mathrm{~h}$. This is shown in Figure 3.10. These long thermalisation times have been noticed previously when a similar CBT with gold electroplated thermalisation blocks was investigated in 


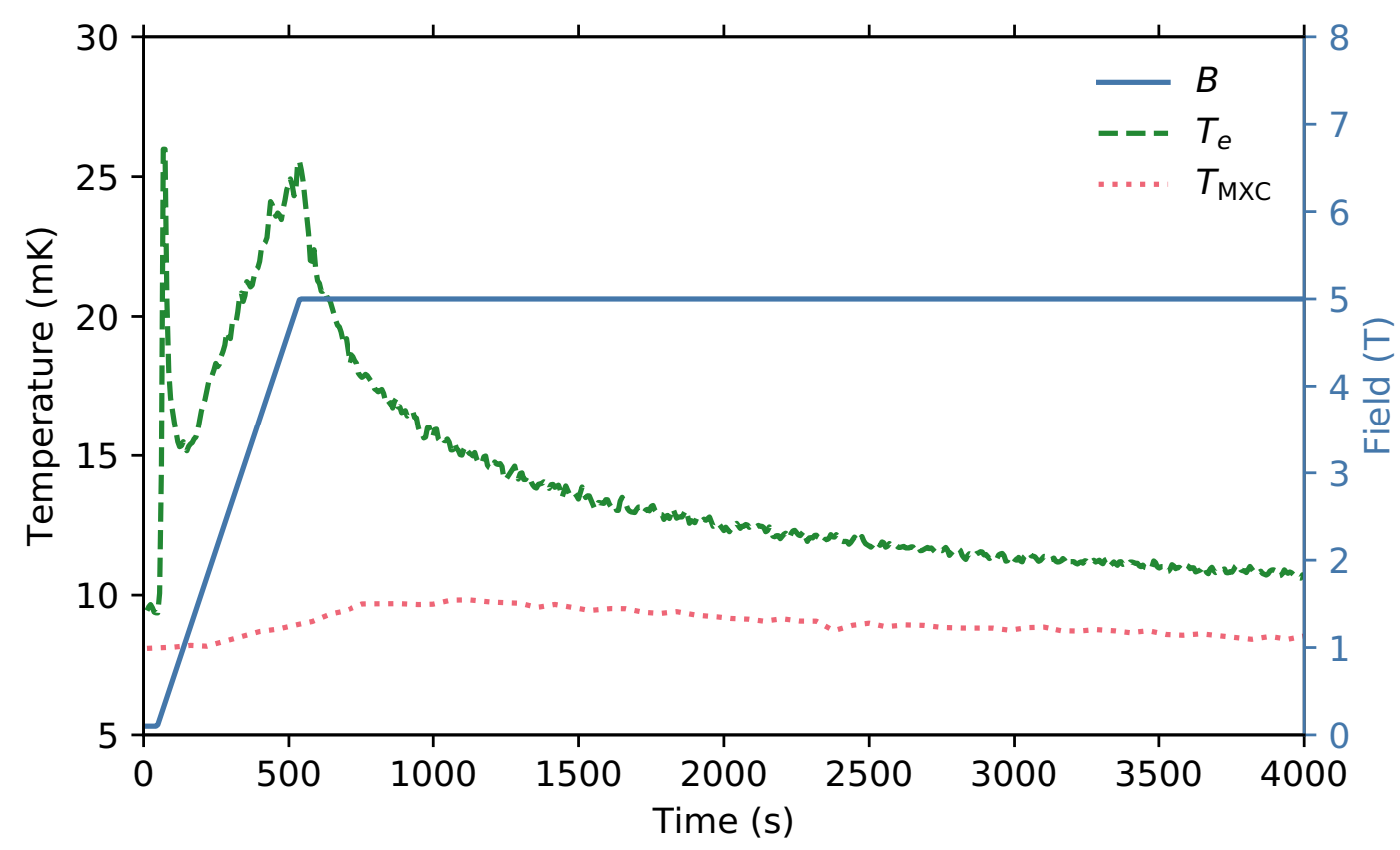

Figure 3.10: CBT and MXC Warming During Magnetisation. As the solenoid is ramped to full field at a constant rate (solid blue line, right axis), the CBT electrons (dashed green line) and MXC plate (dotted red line) warm due to eddy current heating and the heat of magnetisation. The field is then held until they thermalise back to base temperature.

vacuum [49]. The spike in electron temperature near $0 \mathrm{~s}$ is the result of electrical interference when the magnet power supply was energised and does not represent the real temperature, as evidenced by the unrealistic cooling rate immediately afterwards.

With the MXC and CBT thermalised, the magnetic field was swept downwards from $5.0 \mathrm{~T}$ to $0.1 \mathrm{~T}$ at a constant rate of $2.5 \mathrm{mT} \mathrm{s}^{-1}$ while recording the CBT conductance at zero bias at a sample rate of $\approx 6 \mathrm{~s}^{-1}$. During the field sweep, the zero bias conductance was found to decrease by $\approx 15 \%$, as shown by the dashed red line in Figure 3.11. With reference to the calibration curve in Figure 3.8, we can see that this is consistent with $T_{e}$ reducing. It was plausible, however, that the observed conductance change may be caused by, for example, voltages being induced by the changing magnetic flux through the inductors on the filter PCB. To discount this possibility, the exact same demagnetisation procedure was repeated twice more: once at asymptotic bias and once at the point at which the conductance was initially half of its maximum value. As can be seen in Figure 3.11, the conductance at half-maximum bias (green dot-dash line) increases, while the asymptotic conductance (dotted yellow line) is essentially constant at $G_{\text {asym }}=(24.31 \pm 0.03) \mu \mathrm{S}$, a smaller variation than the uncertainty in $R_{T}$ found when characterising the CBT. These observations are consistent with the con- 
ductance dip getting deeper and sharper as the magnetic field decreases, which in turn is consistent with the CBT island electrons cooling. Note that the 'zero bias' curve in Figure 3.11 is actually at a bias of $0.01 \mathrm{mV}$. This is due to the output offset of the preamplifier meaning that the true centre of the conductance dip is measured to be at a slight non-zero DC bias.

Figure 3.12 shows the electron temperatures $T_{e}$ calculated from the zero-bias conductance data presented in Figure 3.11. We see there is a significant reduction in electron temperature from $(9.21 \pm 0.06) \mathrm{mK}$ to $(4.802 \pm 0.011) \mathrm{mK}$ while the MXC chamber temperature remains constant at $(7.97 \pm 0.08) \mathrm{mK}$. This reduction is almost a factor of 2 , and it is to a temperature below that of the dilution refrigerator and hence that of the electrons' host lattice. There is a significant spike in electron temperature towards the end of the demagnetisation which reaches its peak value of $\approx 29 \mathrm{mK}$ at the same time the magnetic field stops ramping. This is the result of eddy current heating while the magnetic field is sweeping, and is explained in more detail in Subsection 3.4.2.

\subsubsection{Thermal Modelling}

To analyse the data in more detail, and hence extract the magnitude of the heat leak into the islands during demagnetisation, we model the cooling process. To do this, we split the CBT island into three linked thermal subsystems: the nuclear spins, which provide the cooling, the electrons, which we measure the temperature of, and the phonon bath, or lattice, which we take to be in thermal equilibrium with entire CBT chip and its associated substrate and package. This arrangement is shown schematically in Figure 3.13 which differs from the passive cooling model shown in Figure 2.4 through the inclusion of the nuclear spin subsystem and the fact that the electron phonon coupling now serves to warm the electrons, rather than cool them.

During demagnetisation cooling, heat from the electrons, which have heat capacity

$$
C_{e}=\frac{\pi^{2}}{2} n R \frac{T_{e}}{T_{F}}
$$

for $R=8.314 \mathrm{~J} \mathrm{~mol}^{-1} \mathrm{~K}$ the ideal gas constant, $T_{F}=8.12 \times 10^{4} \mathrm{~K}$ the Fermi temperature for copper [23] and $n=6.96 \times 10^{-9} \mathrm{~mol}$ the number of moles of copper per island, is transferred to the nuclear spins which have heat capacity

$$
C_{n}=\frac{\lambda n\left(B^{2}+b^{2}\right)}{\mu_{0} T_{n}^{2}}
$$




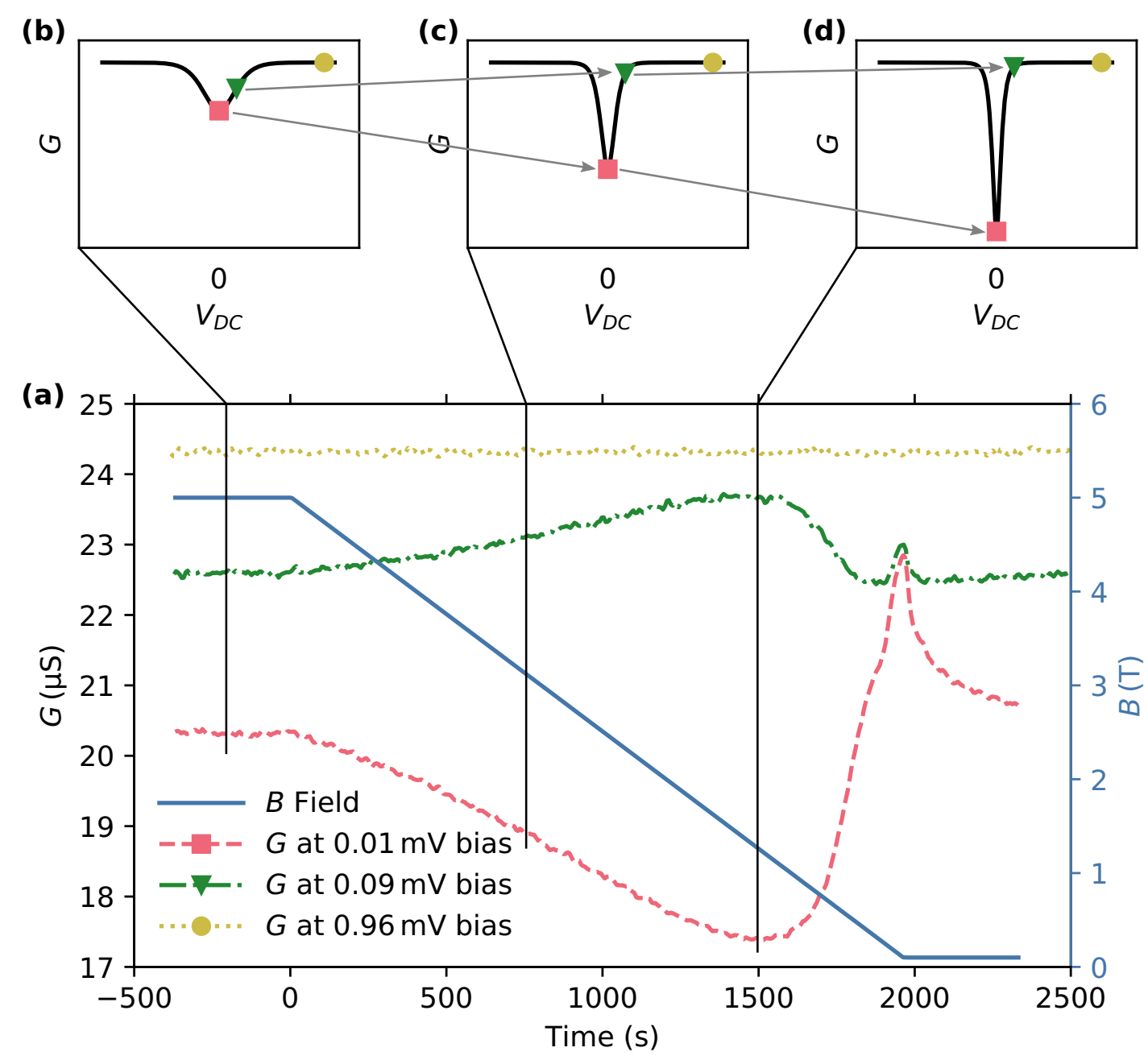

Figure 3.11: CBT Conductance During Demagnetisation at Different Biases. While sweeping the magnetic field down at $2.5 \mathrm{mT} \mathrm{s}^{-1}$ (solid blue line, right axis), from $5.0 \mathrm{~T}$ at $0 \mathrm{~s}$ to $0.1 \mathrm{~T}$, the conductance of the CBT was recorded at zero bias, the bias for initial half-maximum conductance, and asymptotic bias. These are shown in panel (a) by the dashed red, dot-dashed green and dotted yellow lines, respectively. Panels (b)-(d) are schematic CBT conductance curves showing the location of the three bias points as an red square, a green triangle and a yellow circle, respectively. This plot is redrawn from [49]. 


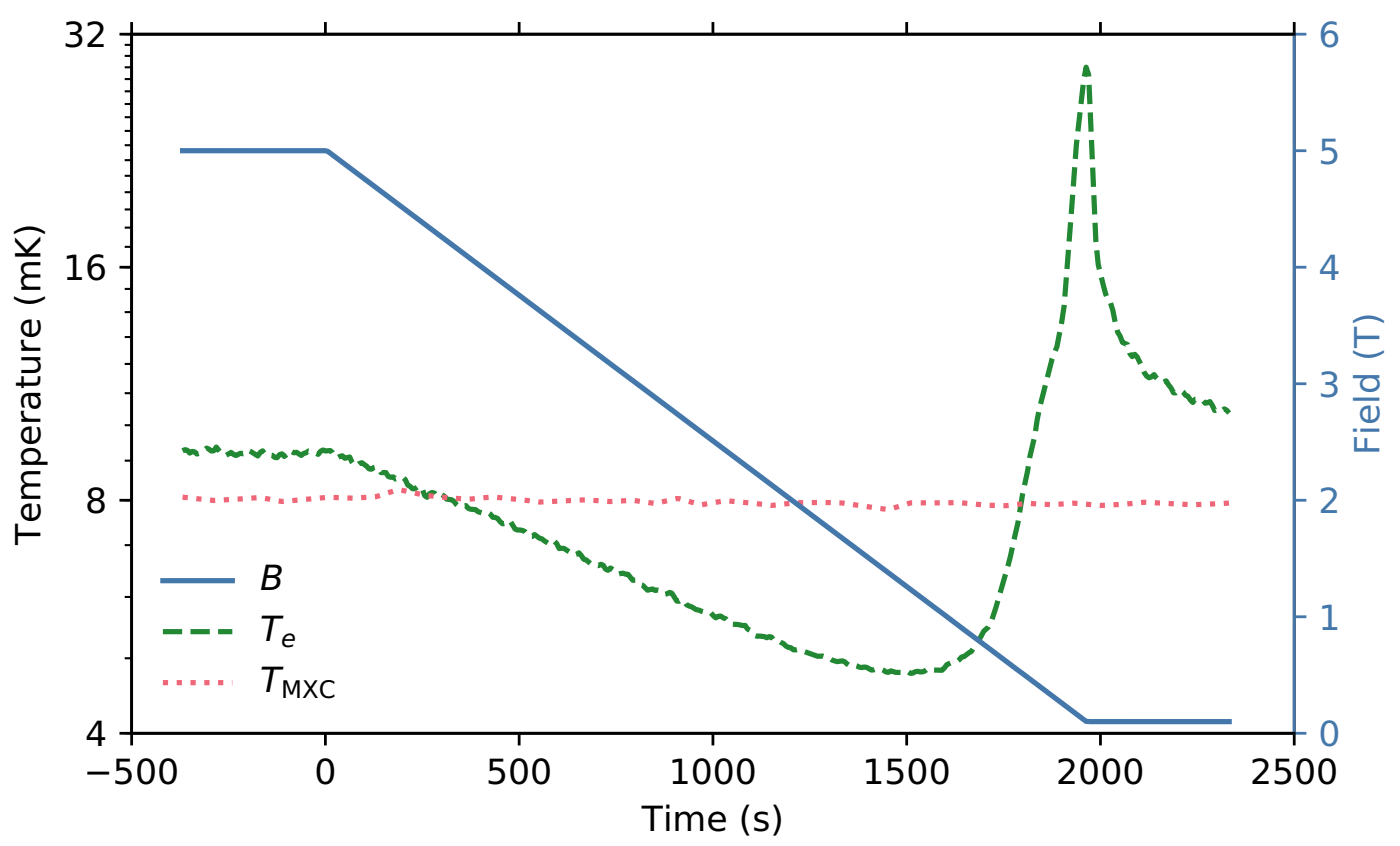

Figure 3.12: CBT Electron Temperature During Demagnetisation From 5.0 $\mathbf{T}$ at $2.5 \mathrm{mT} \mathrm{s}^{-1}$. This shows the zero bias data seen in Figure 3.11 converted to electron temperatures using the self-calibration data in Figure 3.8. For comparison, the MXC temperature is also shown.

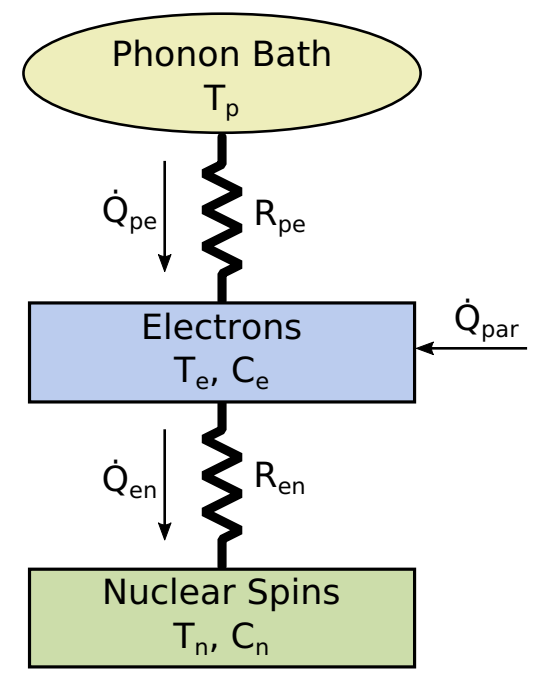

Figure 3.13: Model of the Thermal Subsystems Within a CBT Island. The phonon bath is regarded as having a very large heat capacity due to its thermalisation with the rest of the chip, substrate and sample package. This bath is linked to the electrons, at temperature $T_{e}$ with heat capacity $C_{e}$, which in turn are linked to the nuclear spins, at temperature $T_{n}$ with heat capacity $C_{n}$. An additional heat leak into the electrons is shown as $\dot{Q}_{\mathrm{par}}$. 
for $\lambda$ the nuclear Curie constant, $\lambda / \mu_{0}=3.22 \mu \mathrm{J} \mathrm{K} \mathrm{T}^{-1} \mathrm{~mol}^{-1}$ for $\mathrm{Cu}, B$ the applied magnetic field and $b=0.36 \mathrm{mT}$ the effective internal dipole field in copper [23]. From this, the heat flow from the electrons to the nuclear spin bath can be calculated as (see Subsection 2.1.2)

$$
\dot{Q}_{\mathrm{en}}=\frac{\lambda n\left(B^{2}+b^{2}\right)}{\mu_{0} \kappa T_{n}}\left(T_{e}-T_{n}\right)
$$

for $\kappa=1.2 \mathrm{Ks}$ the Korringa constant for copper [37]. This results in the cooling of the electrons, such that when they are no longer equilibrated with the phonon temperature, the electron-phonon coupling results in a heat flow of

$$
\dot{Q}_{\mathrm{pe}}=\Sigma V\left(T_{p}^{5}-T_{e}^{5}\right),
$$

from the phonons to the electrons, as discussed in Section 2.3. For copper, $\Sigma=$ $2 \mathrm{GW} / \mathrm{m}^{3} \mathrm{~K}^{5}[106,107]$ and here the volume per island is $V=6.14 \mu \mathrm{m} \times 39 \mu \mathrm{m} \times$ $206 \mu \mathrm{m}=4.93 \times 10^{-14} \mathrm{~m}^{3}$.

To model the cooling process, we assume that the nuclear spin subsystem internally reaches equilibrium at a much faster rate than the rate at which thermalisation occurs between the phonons and electrons, and the electrons and nuclear spins. This allows us to simulate the adiabatic demagnetisation as instantaneous, but small, steps down in magnetic field $\delta B$ where the nuclear spin temperature reduces by

$$
\delta T_{n}=T_{n} \frac{\delta B}{B},
$$

since for an ideal adiabatic step $B / T_{n}$ is constant. Following these steps, the relevant amount of time (given the demagnetisation rate) is allowed for heat to flow between the subsystems. In these thermalisation stages, the nuclear spin temperature varies with time $t$ according to

$$
\frac{\mathrm{d} T_{n}}{\mathrm{~d} t}=\frac{\dot{Q}_{\mathrm{en}}}{C_{n}}=\frac{T_{n}}{\kappa}\left(T_{e}-T_{n}\right)
$$

and the electron temperature varies with

$$
\frac{\mathrm{d} T_{e}}{\mathrm{~d} t}=\frac{\dot{Q}_{\mathrm{pe}}+\dot{Q}_{\mathrm{par}}-\dot{Q}_{\mathrm{en}}}{C_{e}}
$$

where additionally $\dot{Q}_{\text {par }}$ is included to represent a parasitic heat leak directly into the island electrons which can arise from, for example, eddy current heating, electronic noise or electromagnetic radiation.

To give numerical clarity to some of these quantities, for a rough example we can 
use some parameters from the $2.5 \mathrm{mT} \mathrm{s}^{-1}$ demagnetisation shown in Figure 3.12. At $1000 \mathrm{~s}, B=3.5 \mathrm{~T}, T_{\mathrm{MXC}}=8 \mathrm{mK}$ and $T_{e}=7 \mathrm{mK}$. If we assume $T_{n}=7 \mathrm{mK}$ here, the highest temperature it can possibly be given the electron temperature, we can calculate that the prefactor of $\dot{Q}_{\mathrm{en}}, \lambda n\left(B^{2}+b^{2}\right) /\left(\mu_{0} \kappa T_{n}\right) \approx 30 \mathrm{pW} \mathrm{K}{ }^{-1}$. When looked at in conjunction with the electron heat capacity $C_{e} \approx 25 \mathrm{fJ} \mathrm{K}^{-1}$ and phonon-electron heat flow $\dot{Q}_{\mathrm{pe}} \approx 1.5 \mathrm{fW}$, we see that the electrons will thermalise very quickly to the nuclear spin temperature, since any deviation from this will lead to a large heat flow from the electrons to the spins, except if the heat leak $\dot{Q}_{\text {par }}$ is extremely large or if the nuclear heat capacity becomes exhausted at low magnetic field.

To verify the model, the electron temperatures shown in Figure 3.12 were fitted to it. To do this, the unknown heat leak $\dot{Q}_{\text {par }}$ was allowed to vary from an initial value of $0 \mathrm{~W}$. Next, the rate of change of the local phonon temperature $\dot{T}_{p}$ was allowed to vary from $0 \mathrm{~K} \mathrm{~s}^{-1}$. This is assumed to take a constant value throughout the demagnetisation, giving a linear increase in phonon temperature as a result of eddy current heating of metallic parts surrounding the CBT chip (e.g. the silver package). Finally, the volume $V$ used to calculate $\dot{Q}_{\text {pe }}$ in Equation 3.8 is decoupled from the number of moles $n$ used to calculate $\dot{Q}_{\mathrm{en}}$ in Equation 3.7 by replacing it with an effective volume $V_{\text {eff }}$. This allows us to account for differences between the heat capacities and densities of the electroplated copper applied to this chip and that used for the calculation of $\Sigma, \lambda$ and $\kappa$. The fitted electron temperatures are shown in Figure 3.14.

We see in Figure 3.14 that this thermal model provides a reasonable fit to the measured electron temperatures, and hence gives us an understanding of how the nuclear temperature $T_{n}$ evolves during the demagnetisation. There is a very small deviation of the measured data from the model at the lowest temperature part of the demagnetisation, where the model predicts a lower electron temperature than is actually observed. This may be the result of electrical noise causing broadening of the conductance curve (Figure 3.5) and hence raised electron temperature measurements. The fitting parameter which controls the relative strength of the electron-phonon coupling, by setting the effective volume that this operates over, is surprisingly low at $12 \%$ of the volume used for the calculation of $\dot{Q}_{\text {en }}$ and $C_{n}$ (via the number of moles $n$ ). This may be accurate for the electroplated copper, particularly if this copper takes a form with a grain size smaller than the mean free path for phonon-electron collisions [108, 109]. Alternatively, this parameter may be forced to take a somewhat unphysical value if the phonon temperature does not rise linearly as we assume here, or if there is a significant deviation from the $T^{5}$ dependence for the electron-phonon coupling, as has been seen in other 


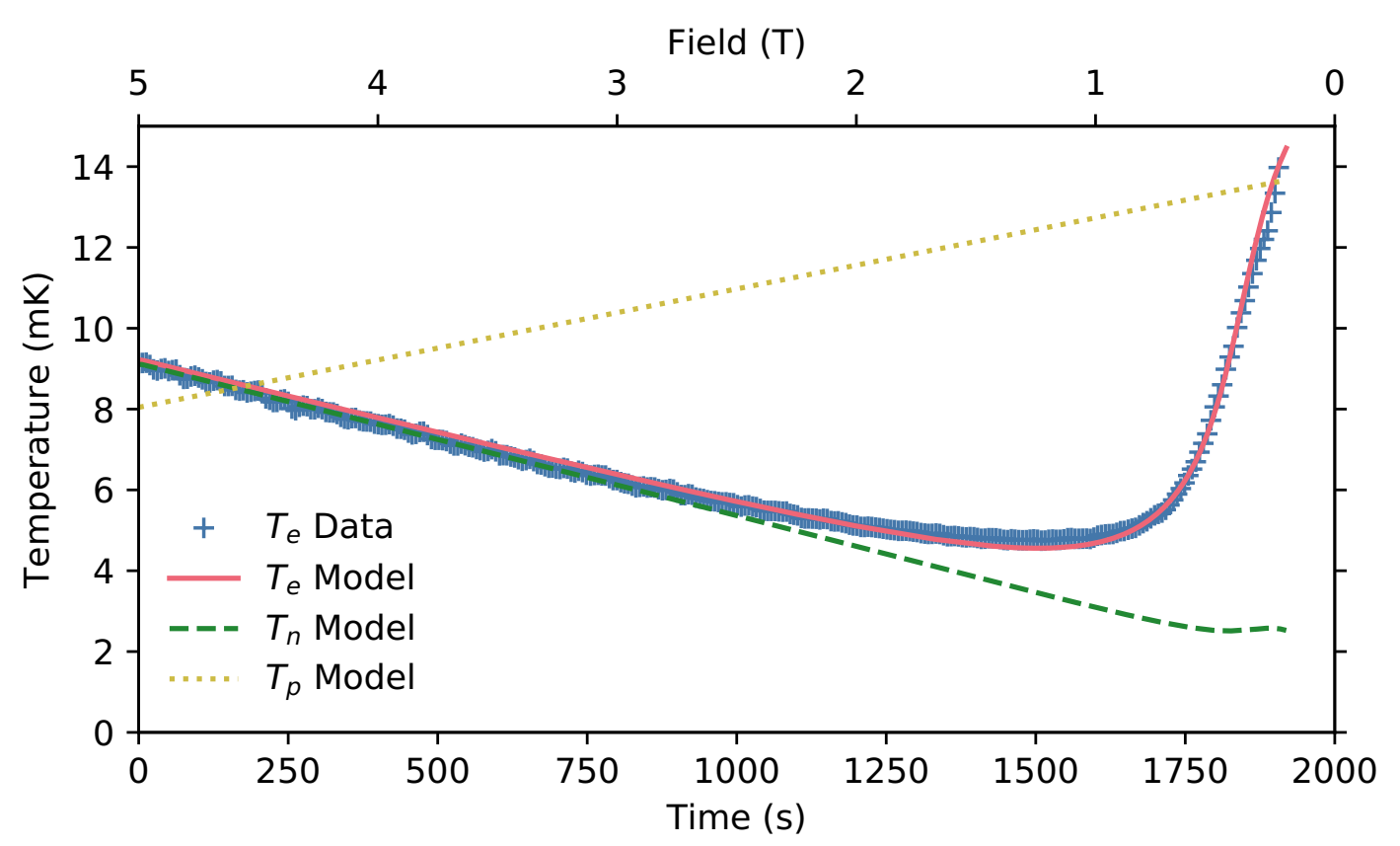

Figure 3.14: Thermal Model Fitted to a $2.5 \mathrm{mT} \mathrm{s}^{-1}$ Demagnetisation. The modelled electron temperature (solid red line) are fitted to the measured data (blue pluses). The fitted parameters were $\dot{Q}_{\mathrm{par}}=6.32 \mathrm{fW}, \dot{T}_{p}=$ $2.96 \mathrm{\mu K} \mathrm{s}^{-1}$ (shown by the yellow dotted line) and $V_{\text {eff }}=0.12 \mathrm{~V}$.

devices $[49,55]$. This model was additionally fitted to four other demagnetisations performed at different rates, and a close agreement between the measured and modelled $T_{e}$ values was found for all cases.

\subsubsection{Optimisation}

We can use information from the thermal model to optimise the demagnetisation process in order to reduce the minimum temperature reached and increase the low temperature hold time. Equation 3.7 shows that the cooling power goes as $B^{2}$, meaning that towards the end of a demagnetisation the cooling power is significantly reduced, enabling the electron-phonon coupling (Equation 3.8), now stronger due to the growing difference between $T_{e}$ and $T_{p}$, and parasitic heat leak to become dominant and hence rapidly heat the electrons. We see this in the model (Figure 3.14), where the electron temperature rises very quickly after $\approx 1500 \mathrm{~s}$, despite the very low nuclear temperature.

To resolve this, the amount of heat that needs to be removed while the magnetic field is small must be reduced. To do this, we note that eddy current heating goes as $\dot{B}^{2}$ [23]. Hence the heat being introduced by eddy currents on the metallic islands themselves, and that introduced indirectly by eddy current heating of the CBT package, can be reduced by slowing down the field ramp rate as smaller 


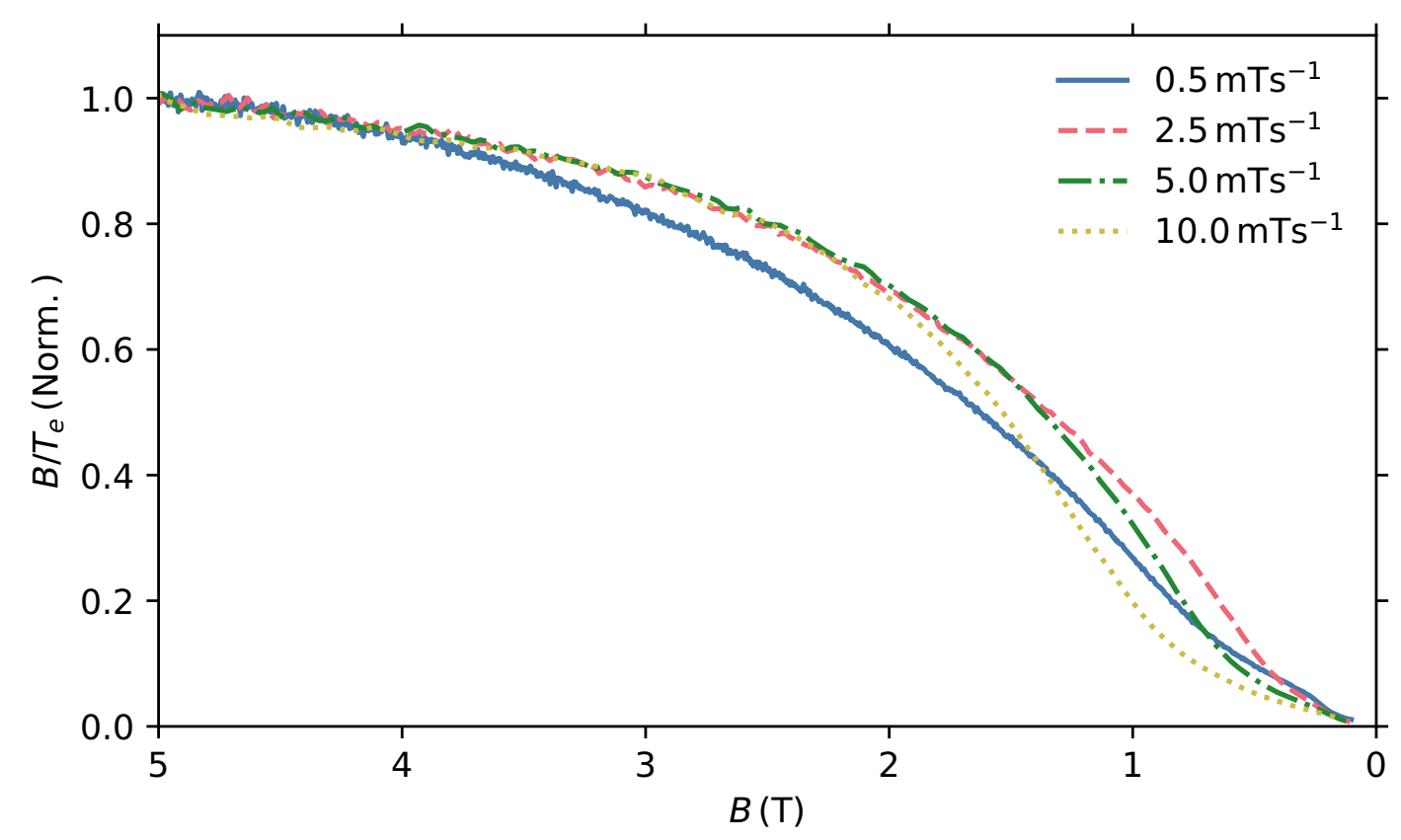

Figure 3.15: Evolution of $B / T_{e}$ Used for Demagnetisation Optimisation. This shows the amount of deviation from the ideal adiabatic case of constant $B / T$ for four different magnetic field sweep rates. Each curve is normalised to 1 at the start of the sweep.

fields are reached. This means that the magnetic field remains higher for longer, allowing more heat to be removed from the electrons while $\dot{Q}_{\text {en }}$ is large, and when the magnetic field is smaller there is less heat input. Additionally, we can see that if the demagnetisation is completely stopped at a higher field, the available nuclear heat capacity (Equation 3.6) is much greater, allowing heat to be absorbed for a longer period after the demagnetisation has stopped and hence increasing the low temperature hold time.

To assist with selecting the appropriate magnetic field sweep rates and the fields as which they should be switched, it is helpful to plot the nuclear entropy during the demagnetisation. The nuclear entropy is entirely a function of $B / T_{n}$, as discussed in Subsection 2.1.2. If we assume that $T_{n}=T_{e}$, which the thermal model in Figure 3.14 shows is true down to $\approx 1 \mathrm{~T}$, then we can say that the entropy is a solely a function of $B / T_{e}$ which is plotted in normalised form in Figure 3.15 for four different magnetic field ramp rates. For an ideal demagnetisation, this plot would show a horizontal line at $B / T_{e}=1$. As such, the deviation from this ideal case can be seen from how far this quantity has reduced.

In Figure 3.15 we see that until $B=2.5 \mathrm{~T}$ there are no significant differences between the $10.0 \mathrm{mT} \mathrm{s}^{-1}, 5.0 \mathrm{mT} \mathrm{s}^{-1}$ and $2.5 \mathrm{mT} \mathrm{s}^{-1}$ sweeps. Therefore we can simply select the fastest rate, $\dot{B}=10 \mathrm{mT} \mathrm{s}^{-1}$, for use until $B=2.5 \mathrm{~T}$ in order to 
produce the fastest possible cool down. Following this, the $10 \mathrm{mT} \mathrm{s}^{-1}$ curve drops away quickly, so instead the $2.5 \mathrm{mT} \mathrm{s}^{-1}$ rate can be chosen from this point. At $1.5 \mathrm{~T}$, while the $2.5 \mathrm{mT} \mathrm{s}^{-1}$ curve still has the greatest value of $B / T_{e}$, the $0.5 \mathrm{mT} \mathrm{s}^{-1}$ curve has the shallowest gradient, hence switching to this rate here then further reduces the entropy change until the end of the demagnetisation. Naively, one may perhaps think that sticking to the slowest possible rate throughout the demagnetisation would be beneficial, however, it is clear from the figure that this incurs an entropy penalty at high fields relative to the other rates. This is particularly the case here, where there is no heat switch to completely isolate the sample from the MXC, since a slow demagnetisation rate means that the rate of heating via the electron-phonon coupling is significant compared with the cooling rate, causing an elevated temperature. Indeed, contrary to most thermodynamic processes, a demagnetisation proceeding at an infinitesimally slow rate would produce no temperature reduction at all here.

The demagnetisation profile (group of magnetic field sweeps rates) described above is shown in Figure 3.16 as the dashed orange line, where the sweep is stopped at $0.1 \mathrm{~T}$, and the dot-dashed green line, where the sweep is stopped at $1.4 \mathrm{~T}$. For comparison, demagnetisation at the constant rates of $10.0 \mathrm{mT} \mathrm{s}^{-1}$ and $2.5 \mathrm{mT} \mathrm{s}^{-1}$ down to $0.1 \mathrm{~T}$ are shown by the solid blue and dotted red lines, respectively. We see that the use of this segmented profile significantly reduces the spike in electron temperature at low magnetic field (note the logarithmic y-axis in Figure 3.16a) and increases the time held below $5 \mathrm{mK}$ from $400 \mathrm{~s}$ (for the constant $2.5 \mathrm{mT} \mathrm{s}^{-1}$ profile) to $1200 \mathrm{~s}$. The base temperature is also slightly reduced relative to the $2.5 \mathrm{mT} \mathrm{s}^{-1}$ profile from $4.7 \mathrm{mK}$ to $4.5 \mathrm{mK}$ and there is a significant reduction in the cooling time. Stopping the sweep at $1.4 \mathrm{~T}$ completely eliminates the peak in electron temperature at low field, and instead allows the CBT to gradually warm up back to the MXC temperature.

\subsection{Conclusions}

In conclusion, we have verified the primary thermometry from a CBT with additional $\approx 6 \mu \mathrm{m}$ thick copper blocks on the metallic islands and found similar behaviour to that seen on a similar device with gold thermalisation blocks [49]. These copper blocks were then used as refrigerant to permit adiabatic nuclear demagnetisation to be performed directly on the chip, and the resulting cooling was verified by using the self-calibrated thermometry of the CBT. By optimising the technique, the electrons on the chip were cooled to a minimum temperature of $4.5 \mathrm{mK}$ and were held below $5 \mathrm{mK}$ for $1200 \mathrm{~s}$. While this was not the lowest 

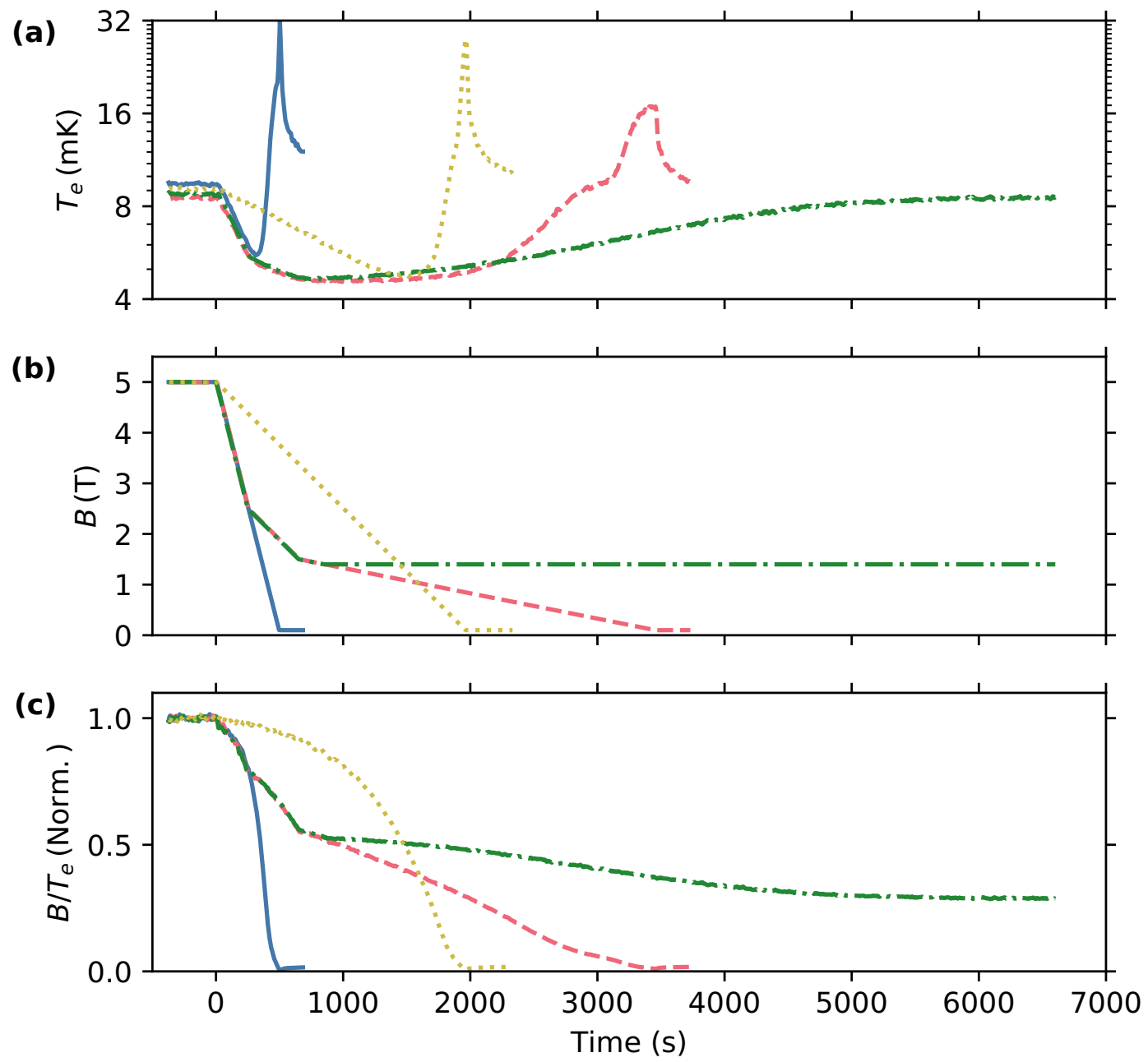

Figure 3.16: Effect of Demagnetisation Profile on CBT Temperature. All parts of this figure are plotted to a common time axis, shown at the bottom of panel (c). Panel (a) shows how the CBT electron temperature depends on the field sweep profile shown in (b). Panel (c) shows the deviation from the ideal case of constant entropy $\left(B / T_{e}=1\right)$. This figure is redrawn from [49]. 
electron temperature obtained in a device at the time, the $3.7 \mathrm{mK}$ result for the immersion cooled CBT [49] being lower, it was a successful proof of concept for the ability to cool the electrons in a device to below their host lattice temperature and significantly below the typically guaranteed base temperature of a commercial dry dilution refrigerator, which is usually around $10 \mathrm{mK}$. 
BLANK 


\section{Chapter 4}

\section{On-Chip Demagnetisation Cooling on a Cryogen-Filled Dilution Refrigerator}

The experiments described in this chapter follow directly from those described in Chapter 3, where on-chip nuclear demagnetisation cooling was performed on a CBT mounted in a dry dilution refrigerator. From the thermal model developed in Chapter 3 (Subsection 3.4.2), it was calculated that, for a system with the same heat leaks but a lower initial electron temperature of $6 \mathrm{mK}$ and higher starting magnetic field of $10 \mathrm{~T}$, a minimum electron temperature of $1.1 \mathrm{mK}$ should be reachable [88]. While we do not possess such a system, we do have a wet dilution refrigerator, designed for very low heat leaks, featuring a $7.5 \mathrm{~T}$ solenoid magnet and a base MXC temperature of $1.75 \mathrm{mK}$ [6]. This chapter describes the use of this system, with the same CBT design as in the previous chapter, in an attempt to reach even lower electron temperatures.

Section 4.1 describes the attachment of the CBT to the wet dilution refrigerator, including detail on the thermalisation techniques required to ensure efficient precooling at much lower temperatures. The CBT used in this investigation is to the same design as that in Chapter 3, however it is not the same chip, hence it was re-characterised and the results of this are given in Section 4.2. More advanced measurement techniques were required for thermometry with this CBT at temperatures around $1 \mathrm{mK}$, so these are also described in Section 4.2 and in the publication [110], which was drafted by the author of this thesis. The results from the demagnetisations, including the minimum electron temperature of $(1.1 \pm 0.4) \mathrm{mK}$, are presented in Section 4.3, and finally the conclusions are drawn 
in Section 4.4.

\subsection{Experimental Set-Up}

Many of the aspects of this experiment are the same as those that were described in Chapter 3. As such, to avoid repetition, this section describes the changes made to the set-up which were necessary as a result of the different experimental environment. In addition, the use of a Lancaster designed wet dilution refrigerator gives a number of benefits in terms of thermalisation of the sensor and of the electrical noise present in the system. The altered aspects concerning the mounting and thermalisation of the CBT are described in Subsection 4.1.1 while the aspects relating to the electrical measurement and environment are given in Subsection 4.1.2. Reference to Section 3.2, where the previous set-up was described, may be beneficial for the reading of this section.

\subsubsection{Mounting and Heatsinking}

With the CBT used in Chapter 3 having demonstrated the on-chip nuclear demagnetisation technique successfully, it was decided to use a second sensor, fabricated on the same $150 \mathrm{~mm}$ diameter silicon wafer and nominally identical to the first. This sensor was attached to a second 3D-printed silver package, again to the same design as the first, using silver epoxy. This was used in place of the silver paste to make the wire bonding process, used for connecting the CBT to the filter PCB, more reliable since the epoxy provides a more solid mechanical connection, while still having high thermal conductivity [111]. The filter PCB integrated into the package was also to the same design, however, this time had bond pads coated with a nickel-free ${ }^{1}$, electroless, immersion coated silver layer, again to aid the wire bonding procedure by improving adhesion of the aluminium bond wires. Before the wire bonding process, the PCB was coated with GE varnish (see Figure 4.1), an electrically insulating varnish with relatively high thermal conductivity at low temperatures [112]. This was applied in order to ensure that the components in the filter are well thermalised, similar to what was done for the Aivon Therma filter in Subsection 3.2.1.

The closed package was mounted on a new design of cold finger suitable for the wet dilution refrigerator being used. This dilution refrigerator has a 'Grenoble Style' plastic MXC [113], the base of which is openable. This allows the top of the cold finger to be mounted directly beneath the MXC, and sealed to it with a cone joint,

\footnotetext{
${ }^{1}$ Nickel-free coating processes are important since nickel is a ferromagnet which may cause undesirable effects during demagnetisations
} 


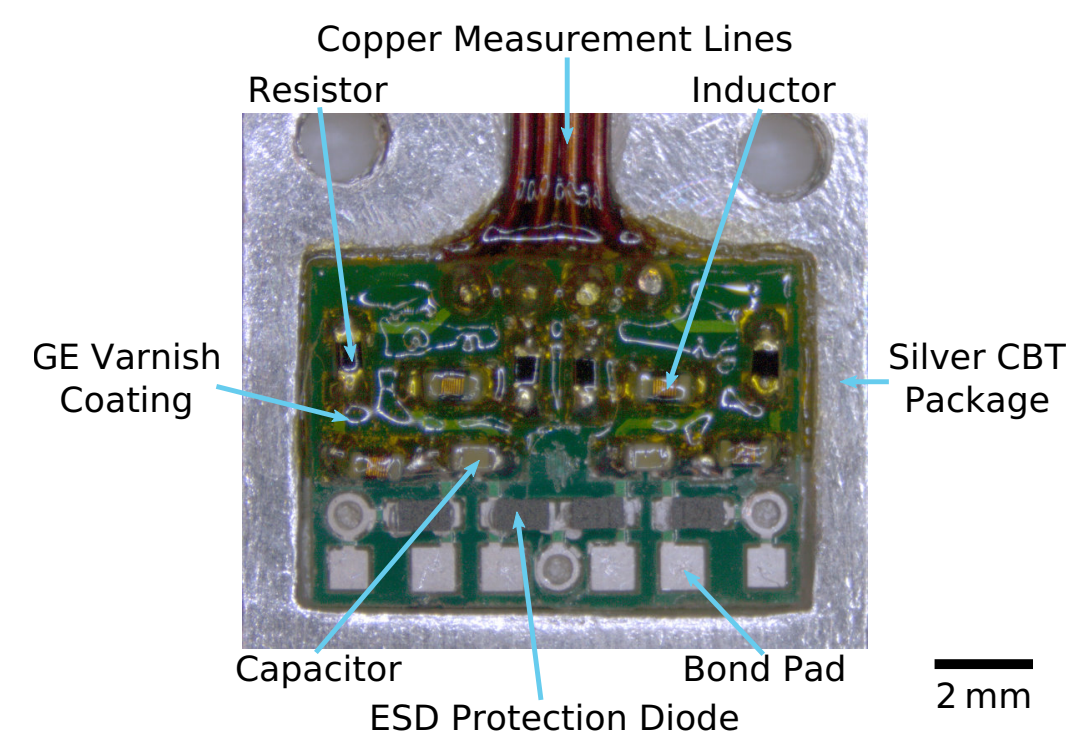

Figure 4.1: CBT Package Filter PCB. This shows the filter PCB, held within the 3D-printed silver package, after the application of the GE varnish. The GE varnish is used to ensure thermalisation of the components on the PCB, and has a translucent yellow appearance.

allowing full experimental access to the ${ }^{3} \mathrm{He}-{ }^{4} \mathrm{He}$ mixture. To minimise the effects of the Kapitza resistance between the mixture and the CBT chip during precooling, the silver sample package and the four measurement lines were directly connected to silver sinters immersed in the helium mixture via high-purity, annealed silver wires, as shown in Figure 4.2. The package is connected to its respective silver wire using the same technique as in Subsection 3.2.1, using a bolt through a silver annulus with a copper washer to ensure it remains tight after thermal contraction. The measurement leads are each connected to a silver wire in a similar manner, by trapping the copper wires, coming from the package, between a nut and a screw passing through a silver annulus, again with a copper washer. Each of the four silver wires used as part of the measurement circuit were insulated with heat shrink and then covered with tinned copper braid, extracted from coaxial cable, in order to provide electrical shielding over their $\approx 20 \mathrm{~cm}$ length. To complete the shielding of these four silver wires, a silver can, featuring many small holes to allow access for the helium mixture, covers the silver sinters.

Above the cone joint, in addition to the silver sinters, there is a plastic 'diagnostic post' used for monitoring the behaviour of the dilution refrigerator. This holds two quartz turning forks, with a nominal resonant frequency of $32 \mathrm{kHz}$, fixed at two different heights and used for sampling the viscosity of the ${ }^{3} \mathrm{He}$ rich (upper) and ${ }^{3} \mathrm{He}$ poor (lower) phases of the ${ }^{3} \mathrm{He}-{ }^{4} \mathrm{He}$ mixture $[114,115]$. There is also a vibrating wire resonator (VWR), made from $124.0 \mu \mathrm{m}$ diameter Ta wire, located in the dilute $\left({ }^{3} \mathrm{He}\right.$ poor $)$ phase for the same purpose $[116,117]$. Both viscometers in the dilute 
(a)

(b)

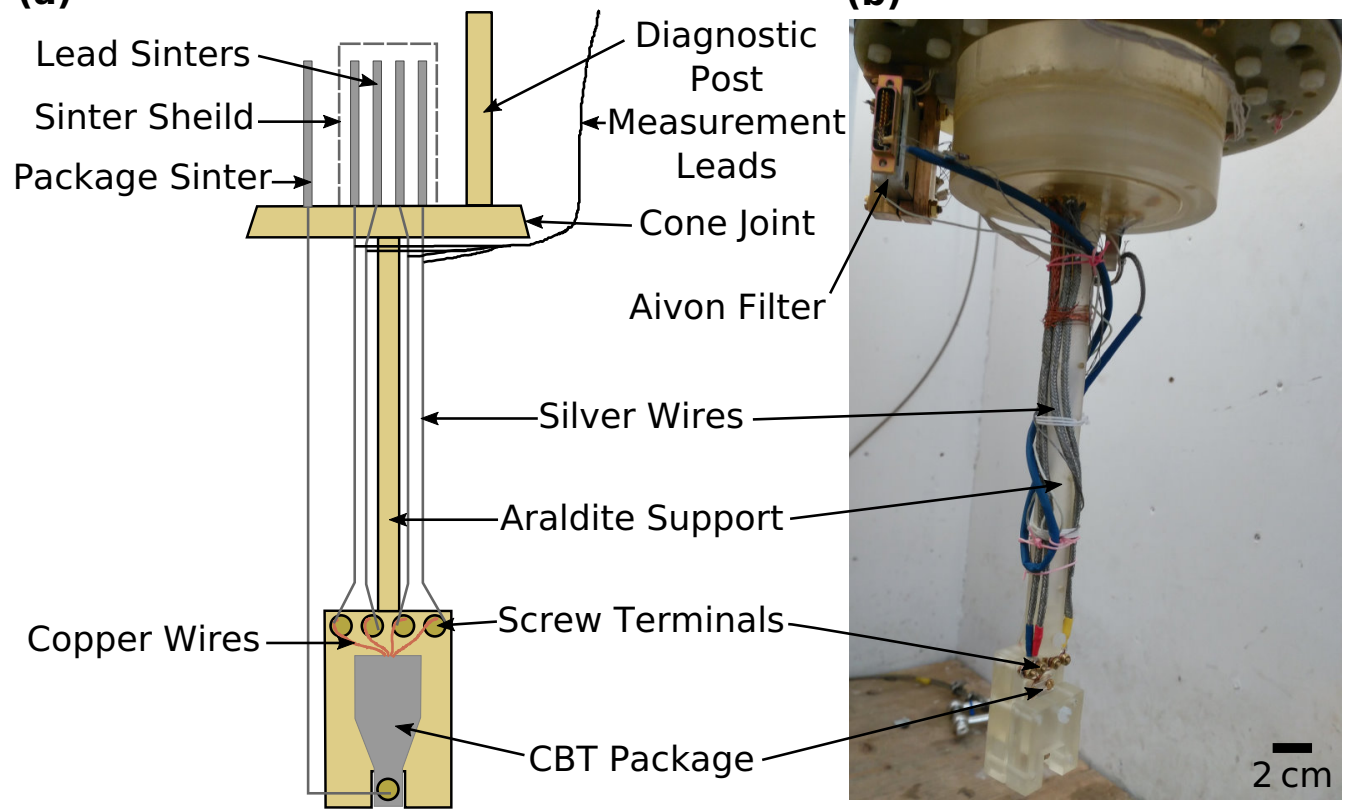

Figure 4.2: CBT Package Mounting. Panel (a) is a schematic drawing of the cold finger shown in the photograph in panel (b). The body of the structure is fabricated from epoxy resin (Araldite ${ }^{\circledR}$ ) and supports the CBT package in a clamp at the bottom. The cone joint seals into the bottom of the MXC, allowing the silver sinters to be immersed in the helium mixture. The length of the support is chosen such that the CBT is at the centre of the bore of the $7.5 \mathrm{~T}$ superconducting magnet.

phase can be used for accurate thermometry of the MXC $[118,119]$. For crude, but fast, thermometry there is a $47 \Omega$ 'Speer' carbon resistor mounted just above the cone which exhibits a strong negative temperature coefficient of resistance at low temperatures [120, 121]. Finally, there is a $100 \Omega$ thin-film resistor mounted at the top of the diagnostic post. This has a small temperature coefficient of resistance, hence can be easily used as a heater to warm the MXC.

The dilution refrigerator used for precooling the sensor is referred to as 'Fridge 6' within the Ultra-Low Temperature Laboratory at Lancaster University. This machine is a copy, with minor improvements, of 'Fridge 5' which has a base temperature of $1.75 \mathrm{mK}$ and a cooling power of $10 \mu \mathrm{W}$ at $20 \mathrm{mK}$, as described in [6], and therefore has a lower base temperature but a comparable cooling power to the Bluefors LD250 used in Chapter 3. This machine was designed and built in Lancaster to have the lowest heat leak reasonably possible in order to achieve the lowest temperatures in adiabatic nuclear demagnetisation experiments, albeit with large bulk copper stages and not small on-chip ones. To this end, the cryostat is located within a room shielded with steel plates in order to create a Faraday cage, preventing the ingress of electromagnetic radiation and electric fields. This also provides some degree of shielding from magnetic fields by means of the fer- 


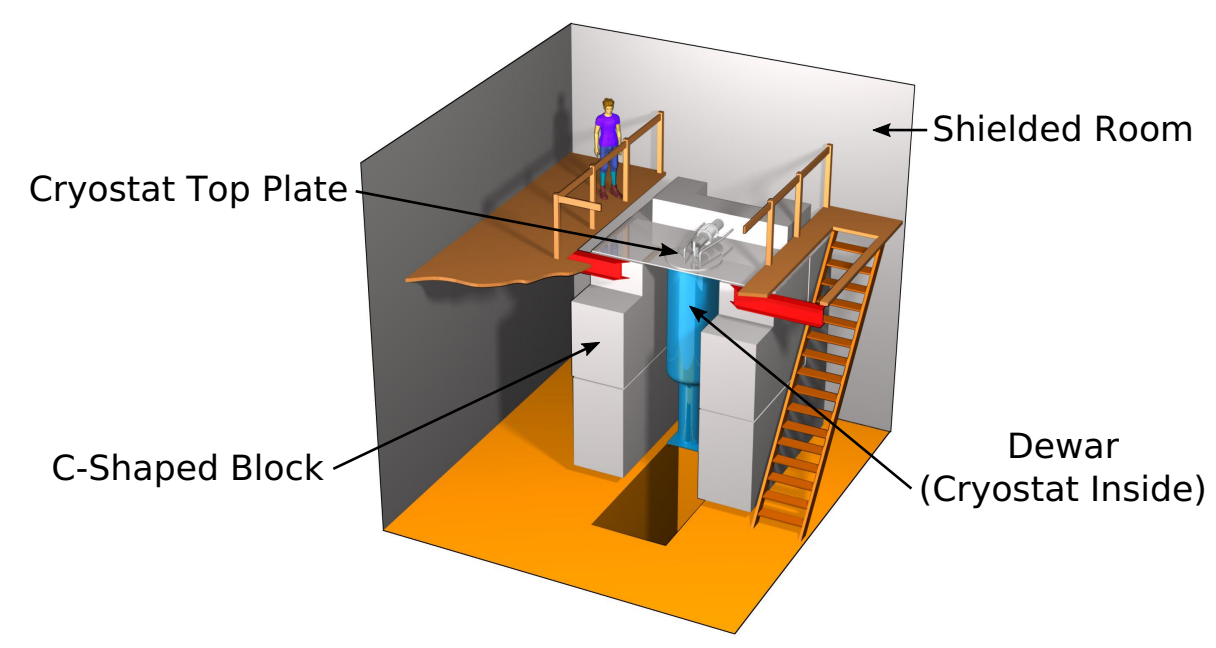

Figure 4.3: Noise Isolation on the Wet Dilution Refrigerator. This is a drawing of the vibration isolation structure, used in Fridges 5 and 6, surrounded by the shielded room. The central graphic was drawn by G.R. Pickett.

romagnetic steel, though this is not as effective as a considerably more expensive Mu-metal shield [122]. The cryostat also features vibration isolation, with the cryostat top plate fixed on top of $50 \mathrm{t}$ of concrete loaded with lead bars and stainless steel re-enforcement. This assembly, in the form of a C-shaped block with the cryostat at the centre of mass (see Figure 4.3), is lifted off the ground by seven air springs which creates a mechanical low pass filter.

\subsubsection{Electrical}

The electrical measurement scheme is similar to that described in Subsection 3.2.2, however, it does feature some changes as a result of the different environment it was used in. The circuit is the same as is shown in Figure 3.4, except that the $\mathrm{PhBr}$ twisted pair measurement lines are replaced with 39 AWG coaxial cables made from non-magnetic stainless steel, each with an end-to-end resistance of $\approx 1 \mathrm{k} \Omega$ and capacitance $\approx 1 \mathrm{nF}$ to ground ${ }^{2}$. Stainless steel has poor thermal conductivity for a metal [23], hence its use for connections between room temperature and the $\mathrm{MXC}$ of a dilution refrigerator is advantageous for the minimisation of heat leaks.

The filtering in the measurement circuit is identical to that used in Chapter 3. As described in Subsection 4.1.1, however, this wet cryostat features significantly more electrical shielding and has a significant vibration isolation structure, c.f. the dry cryostat which does not have such a structure and produces vibrations itself which originate from the pulse-tube cryocooler $[123,124]$. The nominally $230 \mathrm{~V}$ at $50 \mathrm{~Hz}$ mains power supply is filtered at its point of entry into the shielded room, removing

\footnotetext{
${ }^{2}$ This capacitance was only roughly measured using the capacitance function on a multimeter which only had $1 \mathrm{nF}$ resolution.
} 
a significant amount of interference, injected into the mains wiring from equipment elsewhere in the building, which otherwise can couple into the measurement circuit through the mains powered instruments. This filter provides an insertion loss of $100 \mathrm{~dB}$ at $10 \mathrm{kHz}$.

\subsection{CBT Measurements}

The overall method of using a CBT, that has undergone a self calibration procedure in order to perform thermometry of the demagnetisation process at an acceptable sampling rate, is unchanged for this part of the investigation. There are however a number of additional considerations that must be made in order to account for the lower temperatures involved in this part of the study. As a result of the modelling performed at the end of the research in Chapter 3, it was anticipated that we would be working with temperatures around $\approx 1 \mathrm{mK}$, so this temperature was used for the design of the experiment.

The additional measurement techniques required for the use of this CBT at much lower temperatures are described in Subsection 4.2.1. Additionally, since there may be slight differences in the sensor parameters between this device and the nominally identical one measured in Chapter 3, it is important to repeat the self-calibration procedure for the new sensor in the new experimental set-up. This process also ensures that any errors resulting from, for example, different $R_{T}$ values arising from a slightly different amplifier gain, are accounted for in the new self-calibration and hence ensure that the temperature readings obtained are accurate. The results from this new self-calibration are presented in Subsection 4.2.2.

\subsubsection{Additional Techniques}

The CBT used in Chapter 3 has a total capacitance $C_{\Sigma} \approx 200 \mathrm{fF}$, and hence a charging energy (using Equation 2.38) $E_{c} \approx 810 \mathrm{neV}$. This gives $E_{c} / k_{B} \approx 9 \mathrm{mK}$, so the CBT can be operated over the temperature range

$$
3.6 \mathrm{mK}<T_{e}<180 \mathrm{mK}
$$

if a full tunnelling model is used, or

$$
1.8 \mathrm{mK}<T_{e}<180 \mathrm{mK}
$$

if a Monte Carlo simulation of the effects of the island background charges is performed (see Subsection 2.2.3). While this is fine for the measurements at a 
minimum temperature of $4.5 \mathrm{mK}$ in Chapter 3 , and for the $3.7 \mathrm{mK}$ temperatures in the immersion cooling study in [49], for temperatures down to $1 \mathrm{mK}$ it is more problematic.

\section{High charging energy}

When using a CBT with $C_{\Sigma} \approx 200 \mathrm{fF}$ at a temperature of $\approx 1 \mathrm{mK}$, the $1 \sigma$ uncertainty arising from the range of possible background charge distributions is $\approx 40 \%$ (extracted from the simulation data in [50]). When using such a device, there is nothing that can be done about this uncertainty, however, at this point it was unknown if a sufficiently low heat leak could be realised in order to reach temperatures this low using only on-chip nuclear demagnetisation. The only solution to the large uncertainty is to fabricate a new CBT with a larger junction capacitance, so that it has a smaller charging energy. However, developing a new fabrication process, with limited application outside of this project (most tunnel junctions are created for use in SETs or quantum dots where a low capacitance is desirable $[125,126])$, for a cooling technique which was not known to work at these low temperatures, is undesirable. Therefore, it was decided to test the technique using this CBT anyway as a feasibility study.

The $40 \%$ uncertainty quoted above only accounts for the distribution of the CBT island background charges, and does not account for any other errors. Some experimental sources of uncertainty, additional to those encountered in the study in Chapter 3, arise because of the anticipated low temperatures. The first of these relates to the fact the the CBT is being taken away from the intermediate Coulomb blockade regime and towards the strong regime, $E_{c} \gg k_{B} T$. This means that the CBT will start to behave more like a SET, which exhibits full Coulomb blockade. As such, the zero-bias resistance will start to become large $(\sim 1 \mathrm{M} \Omega)$. For the measurement scheme in use for these experiments, a current source applying an $\mathrm{AC}$ excitation of constant amplitude, this results in the creation of large voltages across the sensor.

This can cause issues such as overloaded amplifiers, which can be dealt with as required during the experiment by reducing the gain, but there is a more subtle issue that requires more work to correct. The measurement circuit features three filters, as in Subsection 3.2.2, each having a capacitance between the positive and negative current paths (either directly, or indirectly via ground). The reactance of these capacitances is usually such that the excitation current bypassing the CBT through them is negligible. However, if the CBT resistance becomes much larger, say $500 \mathrm{k} \Omega$, then the capacitor reactance, $\sim 1 \mathrm{M} \Omega$ at $100 \mathrm{~Hz}$, becomes significant. Since the excitation is applied using a current source, this situation 
leads to a smaller than expected current through the CBT, meaning that when the conductance is calculated from the assumed current and measured voltage, the result will be erroneously large.

The solution to this is to perform the measurement at a sufficiently low excitation frequency $f$ such that the capacitor reactance ${ }^{3}$

$$
\left|X_{c}\right|=\frac{1}{2 \pi f C}
$$

for $C$ the capacitance, is high enough so that the excitation current bypassing the $\mathrm{CBT}$ is negligible. While this seems trivial, it is important that this point is realised before measurements commence since the value of $G_{T}$ (asymptotic conductance) may appear to change slightly with frequency as a result of the frequency response of the current source, preamplifier and cryostat wiring. Since this effect scales the entire conductance curve equally, this will not effect the accuracy of the measured electron temperatures provided that the self-calibration procedure is performed at the same frequency as all subsequent temperature measurements. This works because, in primary mode, the temperature is determined by the width of the curve (Equation 2.40), while in pseudo-secondary mode (Equation 2.41), the temperature is determined from the ratio between the magnitude of the conductance curve dip and the asymptotic conductance $\left(\Delta G / G_{T}\right)$. These two quantities are invariant under scaling of the conductance. By analysing a Fourier transform of the signals present in the measurement circuit, it was concluded that $13 \mathrm{~Hz}$ was a suitably low frequency for these measurements which was free from other interference, and hence this was used for all measurements.

\section{Joule heating}

A second issue that arises from the low temperature use of a CBT is the amount of self-heating of the sensor in response to the voltages present across it. This Joule heating, mainly arising from the applied DC bias, is now more significant due to the further reduced heat capacities and cooling powers available at low temperatures. This means that during the self-calibration of the CBT, when the full conductance curves are measured, the outer parts of the curve will be distorted due to the chip being warmed at high bias [49]. To compensate for this, the full tunnelling model calculates the temperature to which the chip is heated at each bias voltage, and then calculates the expected response at this given temperature and bias. It does this by calculating the Joule heating power, which is possible since the voltage

\footnotetext{
${ }^{3}$ There are two conventions for the sign of the reactance: one where capacitive reactance is positive, the other where reactance is the imaginary part of impedance (so capacitive reactance is negative). Here we only concern ourselves with its magnitude.
} 
across the sensor and the sensor's resistance are both known, and then finding the equilibrium temperature using the strength of the electron-phonon coupling (Equation 2.45). This technique was verified to be effective before [49], however it does require that the CBT electrons are in thermal equilibrium when each datum is taken. Since the weak electron-phonon coupling between the electrons and lattice means they warm quickly in response to the Joule heating, but cool slowly, it is fastest to take measurements in an order which only increases the magnitude of the bias amplitude, rather than reducing it and waiting for thermalisation. To this end, the full conductance curves used for self-calibration were measured in two halves: from zero bias to positive bias and, after waiting once for thermalisation, from zero bias to negative bias.

\section{Narrow conductance curve}

A third issue relates to the FWHM of the conductance curve, given approximately by (Equation 2.40)

$$
V_{1 / 2} \approx 5.439 N k_{B} T / e
$$

for $N=33$ the number of tunnel junctions in series. At $1 \mathrm{mK}$, the array used here produces a FWHM of $15 \mu \mathrm{V}$. The non-linearity of the electron temperature dependence on zero-bias conductance, together with this narrow width, means that a small DC bias offset, as little as $100 \mathrm{nV}$ away from zero-bias, can cause a significant pessimistic error in the measured electron temperature. A larger offset, which results in the measurement bias going beyond the FWHM point on the conductance curve, produces more confusing results: The electrons will appear to cool during the demagnetisation, and then warm gradually afterwards as normal. However, at what would be the coldest part of the process, there would appear to be an unphysically fast period of heating followed by unphysically fast cooling (since as the peak sharpens, the conductance outside the half maximum width rises). Note that this issue is not the result of using a CBT with an inappropriately large charging energy, as the FWHM of the conductance curve is directly proportional to temperature for all CBTs. If a completely new CBT was being fabricated, this problem could be alleviated by designing a device with more junctions in series, however, as discussed earlier, we are using the same CBT design as was used in Chapter 3.

To mitigate this issue, two procedures can be used. First, with the sensor thermalised at the base temperature of the dilution refrigerator, a high-resolution and low-noise conductance curve can be recorded in the vicinity of zero-bias. This can then be used to get a reasonable approximation of the true location of the minimum conductance point by fitting a parabola to the data, as shown in Figure 4.4. This 


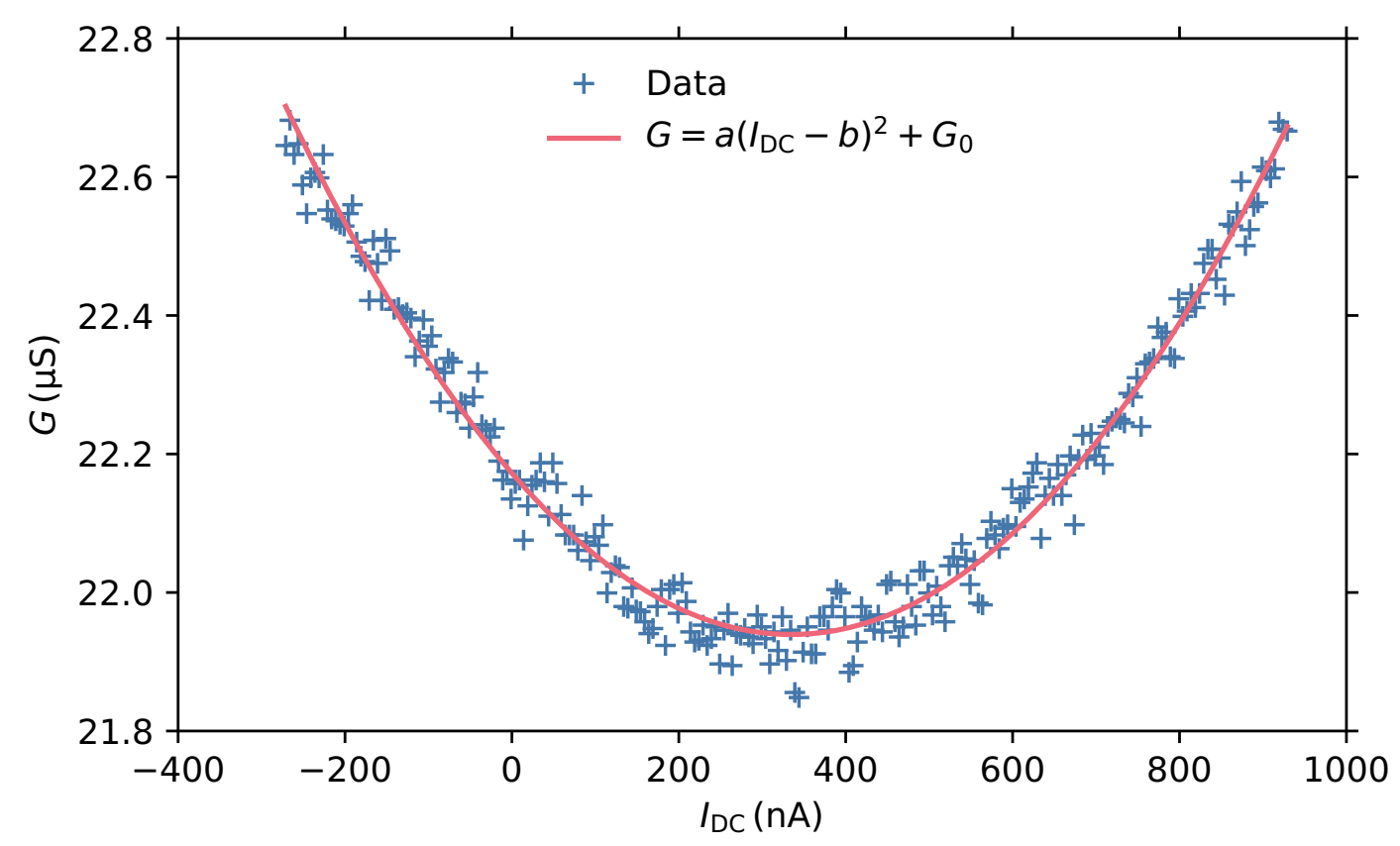

Figure 4.4: Locating the Conductance Minimum at MXC Temperature. The data (blue pluses) was recorded over a small range of DC bias currents at the base temperature of the dilution refrigerator. The fitted parabola (red line) gives the peak centre as $b=(335 \pm 5) \mathrm{nA}$ and minimum conductance $G_{0}=(21.939 \pm 0.005) \mu \mathrm{S}$. The conductances are raised above the base temperature conductances in Figure 4.6 as the solenoid was at $6.8 \mathrm{~T}$, raising the CBT temperature through eddy current heating (see Figure 3.9).

is, however, insufficiently accurate for use at $1 \mathrm{mK}$, since the conductance curve is significantly sharper at this temperature and because the required DC bias can change with time due to drifts in the room temperature equipment caused by the changing room temperature. As a result, in addition to locating the conductance minimum at the base temperature of the dilution refrigerator, it must be tracked throughout the demagnetisation.

The simplest peak tracking technique in principle is to continuously measure small bias conductance curves during the cooling process, fit a parabola to the data, and shift the bias according to the location of this parabola, as has since been performed in other studies [103]. It was decided against this approach due to the short amount of cold time available during the previous demagnetisations and a desire to minimise any excess Joule heating arising from the bias scans. Instead, additional information available at the second harmonic of the excitation frequency was made use of. The functional form of the CBT voltage $(V)$ vs. current $(I)$ curve can be approximated by the third order polynomial

$$
V(I) \approx V_{0}+\alpha I+\beta I^{2}+\gamma I^{3}
$$


for $V_{0}, \alpha, \beta$ and $\gamma$ arbitrary expansion constants. The CBT is driven with an $\mathrm{AC}$ excitation current, at frequency $f$, of the form

$$
I=I_{0} \sin (\omega t)
$$

for $\omega=2 \pi f$ and $t$ time. Hence by substituting Equation 4.6 into Equation 4.5 we find

$$
V \approx V_{0}+\alpha I_{0} \sin (\omega t)+\beta I_{0}^{2} \sin ^{2}(\omega t)+\gamma I_{0}^{3} \sin ^{3}(\omega t)
$$

Expanding the powers of $\sin (\omega t)$ into sums of different harmonics and collecting like terms, we arrive at

$$
\begin{aligned}
V=\left(V_{0}\right. & \left.+\frac{1}{2} \beta I_{0}^{2}\right)+\left(\alpha I_{0}+\frac{1}{2} \gamma I_{o}^{3}\right) \sin (\omega t) \\
& -\frac{1}{2} \beta I_{o}^{2} \cos (2 \omega t)-\frac{1}{6} \gamma I_{0}^{3} \sin (3 \omega t) .
\end{aligned}
$$

Hence, we can measure the DC component

$$
V_{D C}=V_{0}+\frac{1}{2} \beta I_{0}^{2}
$$

the in-phase AC component at frequency $f$

$$
X_{f}=\alpha I_{0}+\frac{1}{2} \gamma I_{o}^{3}
$$

and the out-of-phase (due to the cos function) AC component at frequency $2 f$

$$
Y_{2 f}=-\frac{1}{2} \beta I_{o}^{2}
$$

These components are shown for a simulated CBT in Figure 4.5.

As can be seen in Figure 4.5, the component at frequency $2 f$ is zero at zero bias, positive for positive bias and negative for negative bias. With the Signal Recovery 7265 lock-in amplifier in dual-harmonic mode, this signal at double the frequency can be measured in parallel with the main data collection. This information can be used in a feedback loop which adjusts the applied DC bias such that the measurement remains centred at the minimum of the conductance dip, i.e. where the double frequency signal is minimised. Note, however, that the signal at $2 f$ is considerably smaller in magnitude than that at $f$, so in order to successfully measure it, a long time constant is required on the lock-in amplifier. This means the rate of corrections applied by the feedback loop is slow, and therefore the dip location must be roughly correct before using this technique. This was ensured by first finding the centre at the base temperature of the dilution refrigerator, as described 


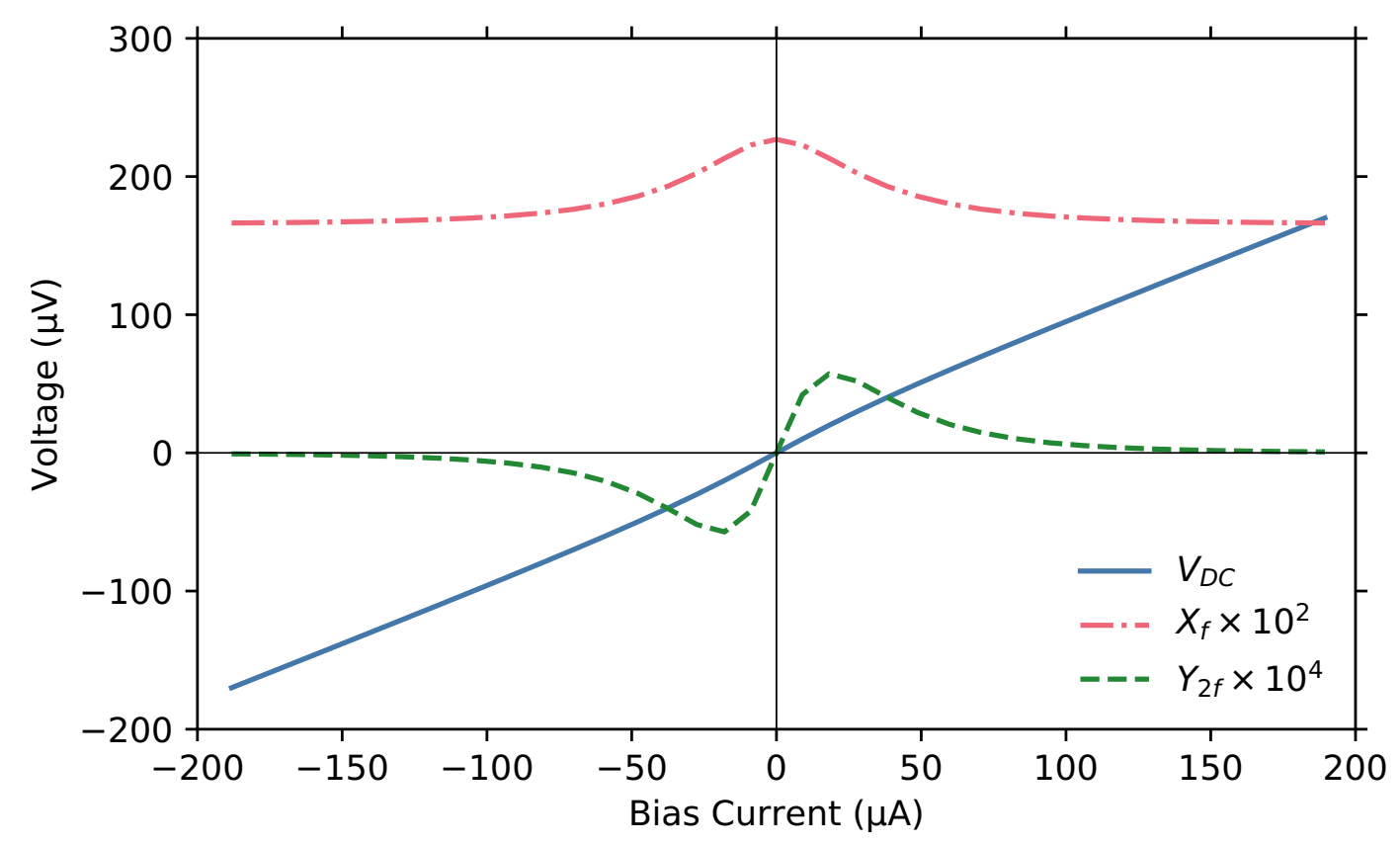

Figure 4.5: Frequency Components of the Signal from a Simulated CBT. A CBT biased with a DC current and measured with an AC excitation current at frequency $f$ results in, to second order, a DC voltage $V_{\mathrm{DC}}$, an in phase $\mathrm{AC}$ voltage $X_{f}$ at frequency $f$ and an $\mathrm{AC}$ voltage $Y_{2 f}$ at frequency $2 f$ with a $\pi / 2$ phase shift. This figure was used in a redrawn form in [110].

above, before applying this procedure.

In addition to the signal at the second harmonic which we use for peak locating, we note that the signal measured at the first harmonic (Equation 4.10) does not exactly yield the differential conductance when divided by $I_{0}$ (as it would for a linear current-voltage characteristic). Hence, in the CBT simulation used in the full tunnelling model, clusters of a small number of adjacent data points are fitted to a cubic polynomial (Equation 4.5), allowing the extraction of the coefficients $V_{0}, \alpha, \beta$ and $\gamma$ at several locations along the curve. These coefficients are then used in Equation 4.10 to calculate the voltages present at frequency $f$ which are then fitted to the real CBT data by varying $C_{\Sigma}, R_{T}$ and $T_{e}$.

Finally, with such a narrow peak at low temperatures, the issues concerning the broadening of the conductance curve by noise or excessive excitation, as described in Subsection 3.2.2 (Figure 3.5), are now more significant. The operation of the CBT in the low noise environment provided by this dilution refrigerator, as discussed in Subsection 4.1.1, inherently deals with the former problem. However, it was important that the excitation current was sufficiently small. To this end, an excitation current of $10 \mathrm{pA}$ was used, which required a lock-in time constant of $10 \mathrm{~s}$ in order to achieve an acceptable SNR in the final data. 


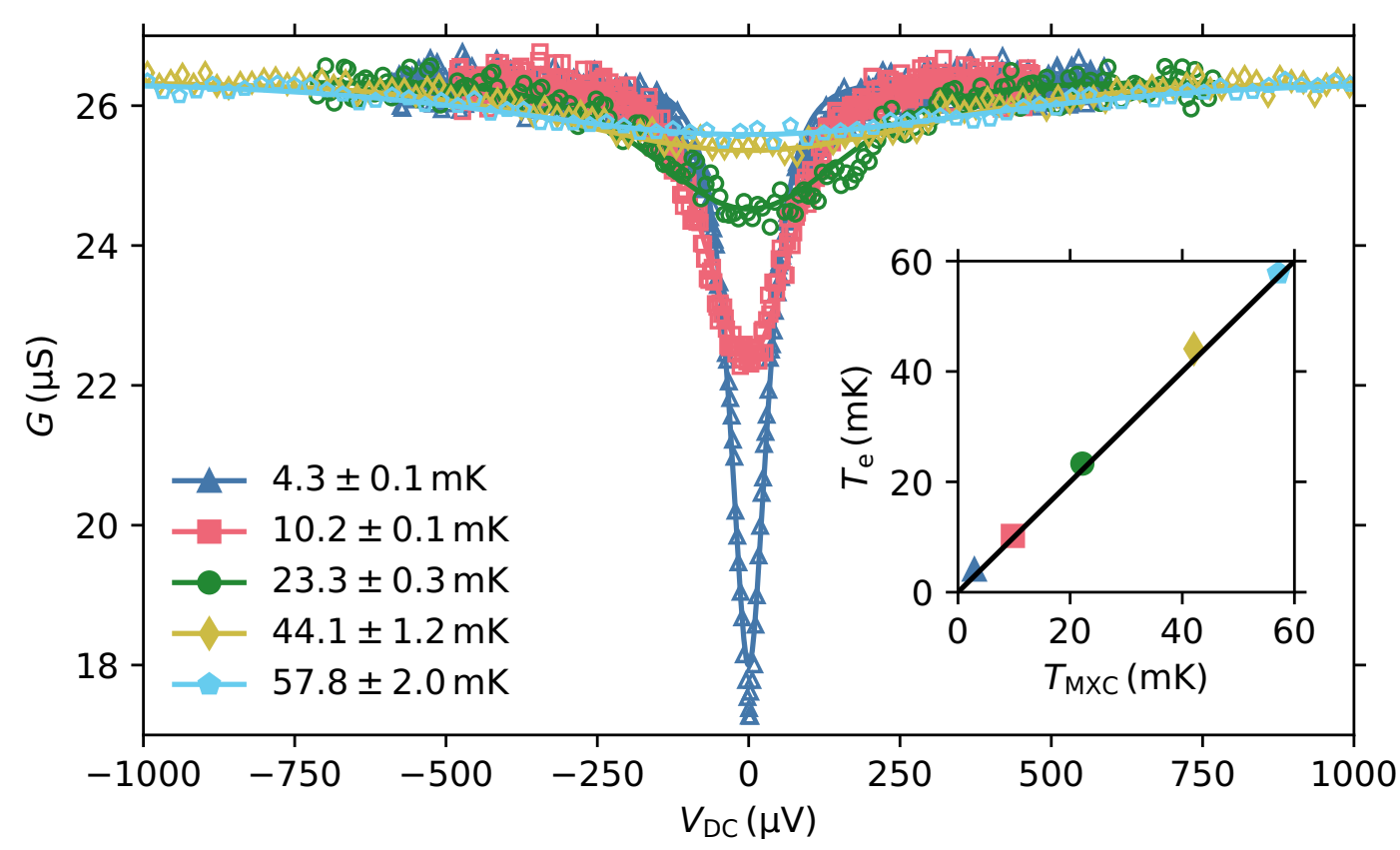

Figure 4.6: CBT Conductance Curves. The symbols show the measured values and the lines show the fits to a full tunnelling model, from which the temperatures shown in the legend are extracted. The inset shows the extracted electron temperature against the temperature the mixing chamber was heated to. The solid line in the inset is $T_{e}=T_{\mathrm{MXC}}$ for reference. This data was measured at a field of $100 \mathrm{mT}$.

\subsubsection{Characterisation Results}

Once the CBT was cooled in the dilution refrigerator, five full conductance curves were measured at different temperatures, as shown in Figure 4.6. As before, each temperature was achieved by heating the MXC using the resistive heater mounted on the diagnostic post inside the MXC (Figure 4.2). The resulting temperature of the helium mixture was then recorded by using the lower tuning fork to measure the viscosity. Given that it had already been established that the thermometry of this design of CBT is not sensitive to magnetic field (Section 3.1), this procedure was only performed at a field of $100 \mathrm{mT}$. The full tunnelling model, with the corrections applied to account for Joule heating and the actual signal detected at the excitation frequency, as described in Subsection 4.2.1, was then fitted simultaneously to the warmest four conductance curves. This gave the warmest four electron temperatures and the CBT sensor parameters, which are shown in Table 4.1. Although the CBT measured in Chapter 3 was nominally to the same design as this one, and was fabricated on the same silicon wafer as part of the same batch, there is a $3 \%$ difference in $C_{\Sigma}$ and an $8 \%$ difference in $R_{T}$ between the two sensors. 


\begin{tabular}{ccc}
\hline Dilution & \multicolumn{2}{c}{ CBT Parameter } \\
\cline { 2 - 3 } refrigerator & $C_{\Sigma}(\mathrm{fF})$ & $R_{T}(\mathrm{k} \Omega)$ \\
\hline Wet & $185.7 \pm 1.0$ & $23.015 \pm 0.004$ \\
Dry & $191.1 \pm 0.6$ & $25.05 \pm 0.04$ \\
\hline
\end{tabular}

Table 4.1: CBT Sensor Parameters. The parameters measured using this CBT on the wet dilution refrigerator were extracted from the fit to the full tunnelling model, as shown in Figure 4.6. For comparison, the average sensor parameters found in Chapter 3 on the dry dilution refrigerator (using the first CBT) are shown on the row underneath.

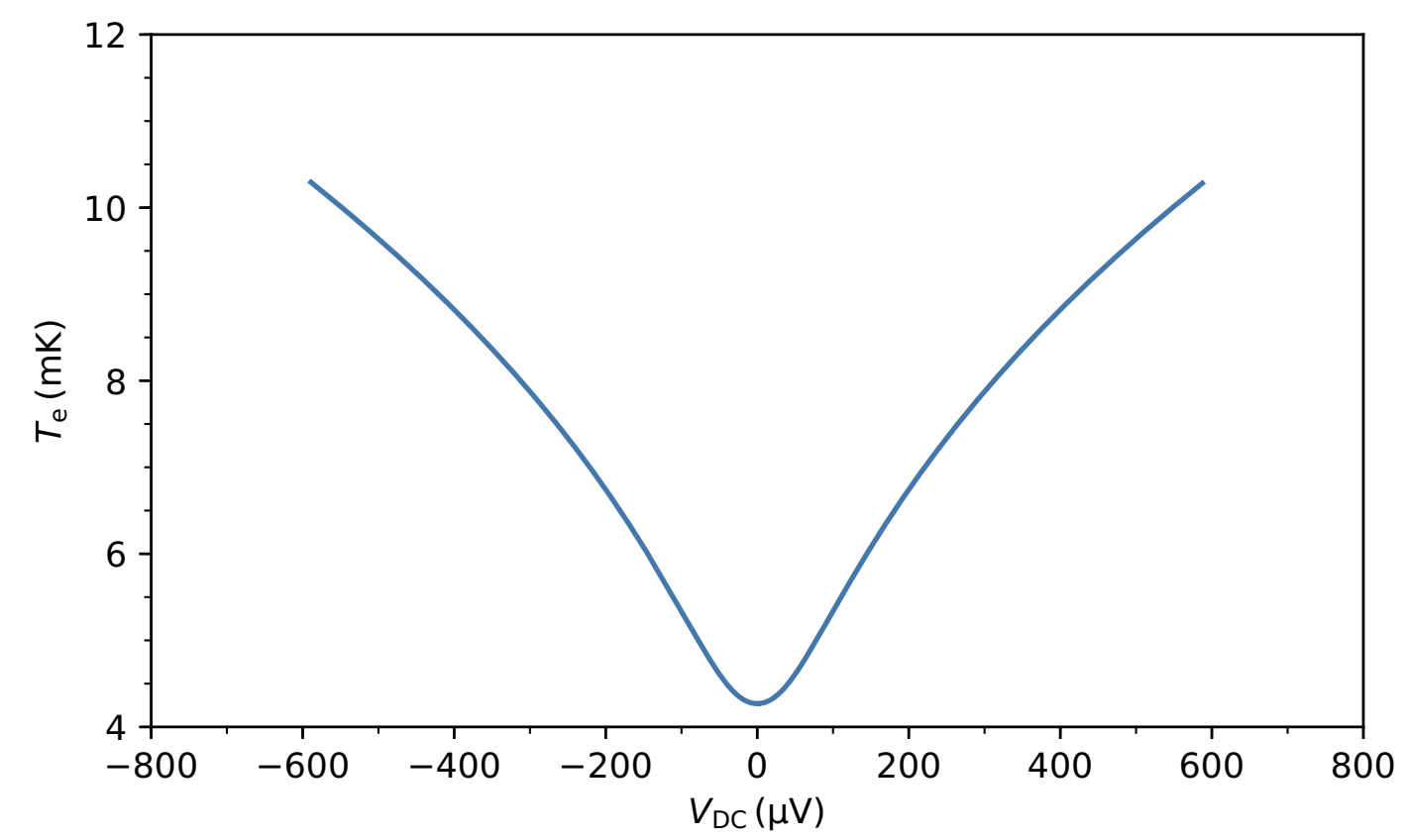

Figure 4.7: Joule Heating During a CBT Conductance Curve Measurement. This shows the predicted electron temperatures in the CBT islands when measuring the $4.3 \mathrm{mK}$ conductance curve shown in Figure 4.6.

As discussed in Subsection 4.2.1, the full tunnelling model used to fit the conductance data shown in Figure 4.6 accounts for the Joule heating which occurs when a DC bias is applied to the sensor. Figure 4.7 shows the simulated temperatures used for this compensation on the fit of the lowest temperature $(4.3 \mathrm{mK})$ conductance curve. This clearly shows the weakness of the electron phonon coupling, as at $V_{\mathrm{DC}}= \pm 600 \mu \mathrm{V}$, where the Joule heating per island is $14 \mathrm{fW}$, the electron temperature is raised by $\approx 6 \mathrm{mK}$. Although it is not clear from the inset of Figure 4.6, during the measurement at the base temperature of the dilution refrigerator, the CBT electrons at $T_{e}=(4.3 \pm 0.1) \mathrm{mK}$ were significantly overheated from the MXC temperature of $T_{\mathrm{MXC}}=(2.9 \pm 0.2) \mathrm{mK}$, even after this compensation.

As before, the measured CBT parameters were used with the full tunnelling model 


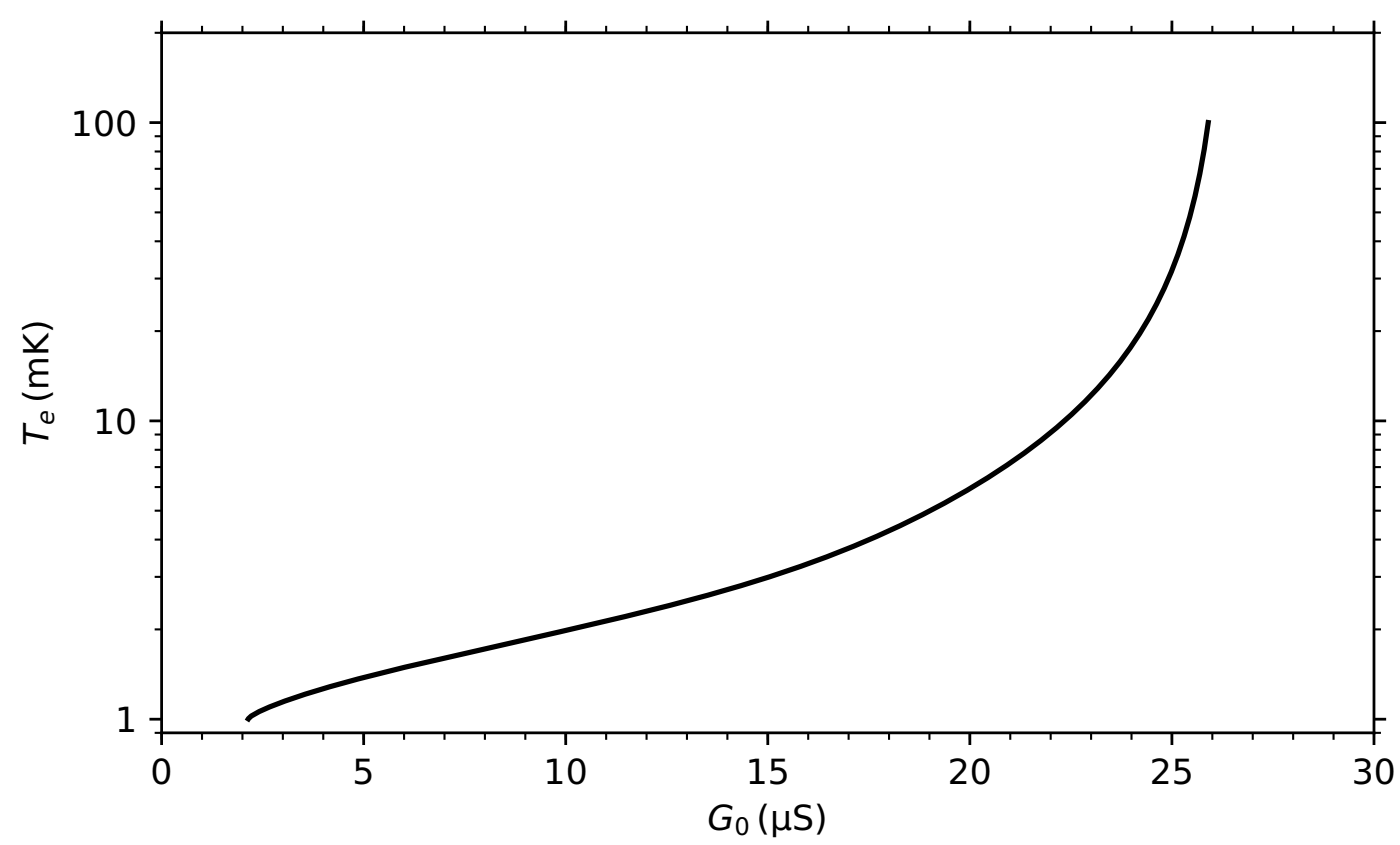

Figure 4.8: CBT Temperature vs. Conductance Self-Calibration Curve. The data in this plot was used to calculate the CBT's electron temperature $T_{e}$ from a measurement of the zero bias conductance $G_{0}$.

to compute a lookup table relating the zero-bias conductance to the electron temperature. This is plotted graphically in Figure 4.8, and allows much faster measurements of the electron temperature to be made than if full conductance curves were to be measured.

\subsection{Demagnetisation Cooling}

Prior to each demagnetisation experiment, the superconducting solenoid magnet was ramped up to a field ${ }^{4}$ of $6.8 \mathrm{~T}$ over a period of $\approx 1 \mathrm{~h}$. Following this, the CBT electrons were allowed to thermalise. The magnetisation and cooling curve is similar to Figure 3.10, except that the thermalisation time is now much longer at $\approx 70 \mathrm{~h}$ to achieve an electron temperature of $6 \mathrm{mK}$, although this could be considerably reduced to $\approx 20 \mathrm{~h}$ which yielded an electron temperature of $8 \mathrm{mK}$. These longer cooling times at low temperatures are consistent with the thermalisation time measurements for a CBT in vacuum in $[49,127]$. Once thermalisation was achieved, the magnetic field was swept down while measuring the CBT conductance every $20 \mathrm{~s}$ and updating the DC bias, in order to remain centred on the conductance dip, every $10 \mathrm{~s}$. The longer measurement interval, as compared to the measurements on the dry dilution refrigerator, is the result of the smaller excit-

\footnotetext{
${ }^{4}$ While this solenoid has a nominal maximum field of $7.5 \mathrm{~T}$, it was found in practice that a maximum of $6.8 \mathrm{~T}$ was needed to ensure stability of the magnet in persistent mode.
} 
ation current requiring a longer lock-in time constant in order for an acceptable SNR to be achieved in the final data.

A $2.7 \mathrm{mT} \mathrm{s}^{-1}$ demagnetisation is shown in Figure 4.9. Figure 4.9a shows the temperature variation, and reports a minimum electron temperature $(1.1 \pm 0.4) \mathrm{mK}$. Note, the large uncertainty is a result of using this CBT, with a relatively large charging energy, outside of its normal operation range, see Subsection 4.2.1. Figure $4.9 \mathrm{~b}$ shows the action of the feedback algorithm used to center the measurement at the minimum of the conductance dip, as described in Subsection 4.2.1. In this, the signal at the second harmonic is approximately zero initially, since any offset present was small as a fraction of the dip width at $\approx 6 \mathrm{mK}$. As the CBT cools, however, the offset becomes more significant as the conductance dip sharpens. This leads to the feedback algorithm responding by reducing the bias, overshooting slightly, then bringing the bias up to its optimal value. To reduce this overshooting a proportional-integral-differential (PID) [128] algorithm was tested, however, it was found that the sample rate of the error signal (the second harmonic) was too slow for the integral and differential components of this to work well, and the fact that the error signal magnitude increases as the CBT cools meant that the proportional component tended to cause overshoots. Instead, a simple algorithm was used which made a fixed correction to the bias for each error signal over a threshold.

This $2.7 \mathrm{mT} \mathrm{s}^{-1}$ demagnetisation was fitted to the same thermal model as described in Subsection 3.4.2, as shown in Figure 4.10. It is clear immediately that while the cooling down to $T_{e} \approx 2 \mathrm{mK}$ looks reasonable, the fit is poor at the lowest temperatures. The model predicts lower electron temperatures than were measured, an earlier turning point after which the electron temperature begins to rise, and a static phonon temperature $\left(\dot{T}_{p}=0.00 \mu \mathrm{K} \mathrm{s}^{-1}\right)$. While the static phonon temperature initially appears reasonable, since there is a much smaller volume of metal in this dilution refrigerator available to cause eddy current heating, the measured MXC temperature increased to $4.1 \mathrm{mK}$ by $2500 \mathrm{~s}$, suggesting that the rate of phonon heating should be at least $\approx 0.5 \mu \mathrm{K} \mathrm{s}^{-1}$. The effective volume is also extremely different from that found in the previous device in Subsection 3.4.2: $96 \%$ of the true volume vs. $12 \%$. The heat leak $\dot{Q}_{\text {par }}=4.76 \mathrm{fW}$ appears reasonable, particularly when compared with the slightly larger heat leak achieved on the dry dilution refrigerator as expected, although given the poor fit, none of the parameters should be regarded as accurate. The poor fit is most likely the result of inaccurate electron temperatures arising from operating this CBT too cold.

Despite the poor fit to the model, the optimisation procedure used in Subsec- 

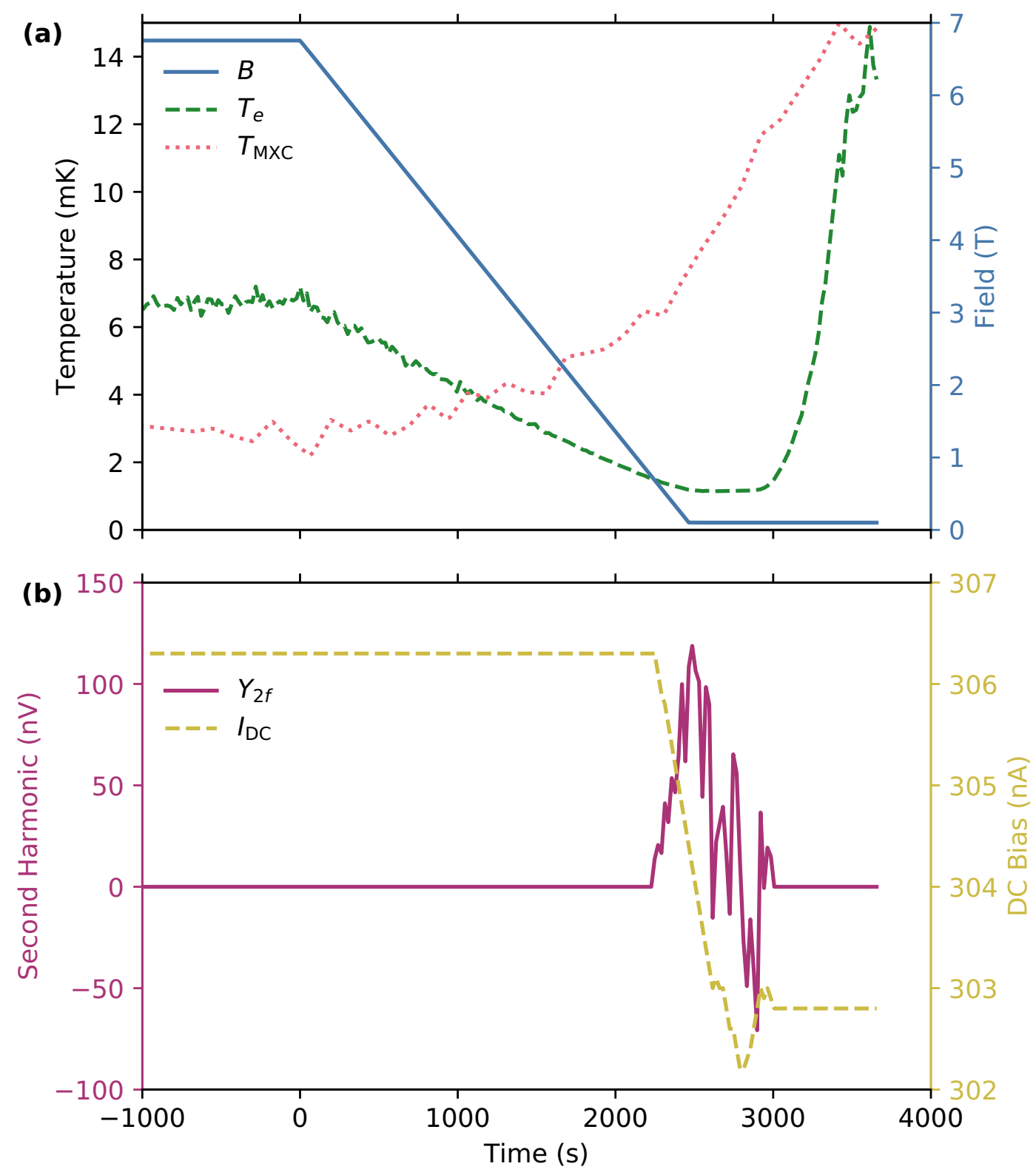

Figure 4.9: Demagnetisation at $2.7 \mathrm{mT} \mathrm{s}^{-1}$. Panel (a) shows the variation in the temperature (left axis) of the CBT electrons (green dashed line) and MXC (red dotted line) in response to the magnetic field variation (solid blue line, right axis). Panel (b) shows the signal measured by the lock-in at twice the excitation frequency $\left(Y_{2 f}\right.$, solid purple line, left axis) and the change in $\mathrm{DC}$ bias $\left(I_{\mathrm{DC}}\right.$, yellow dashed line, right axis) the feedback algorithm causes in response to this. The $I_{\mathrm{DC}}$ values quoted here are the nominal currents applied by the current source, and are not corrected to be relative to true zero bias. Due to a bug in the feedback program, the second harmonic was not recorded if no change to the DC bias was made, hence it is shown here as being nominally zero at these points. 


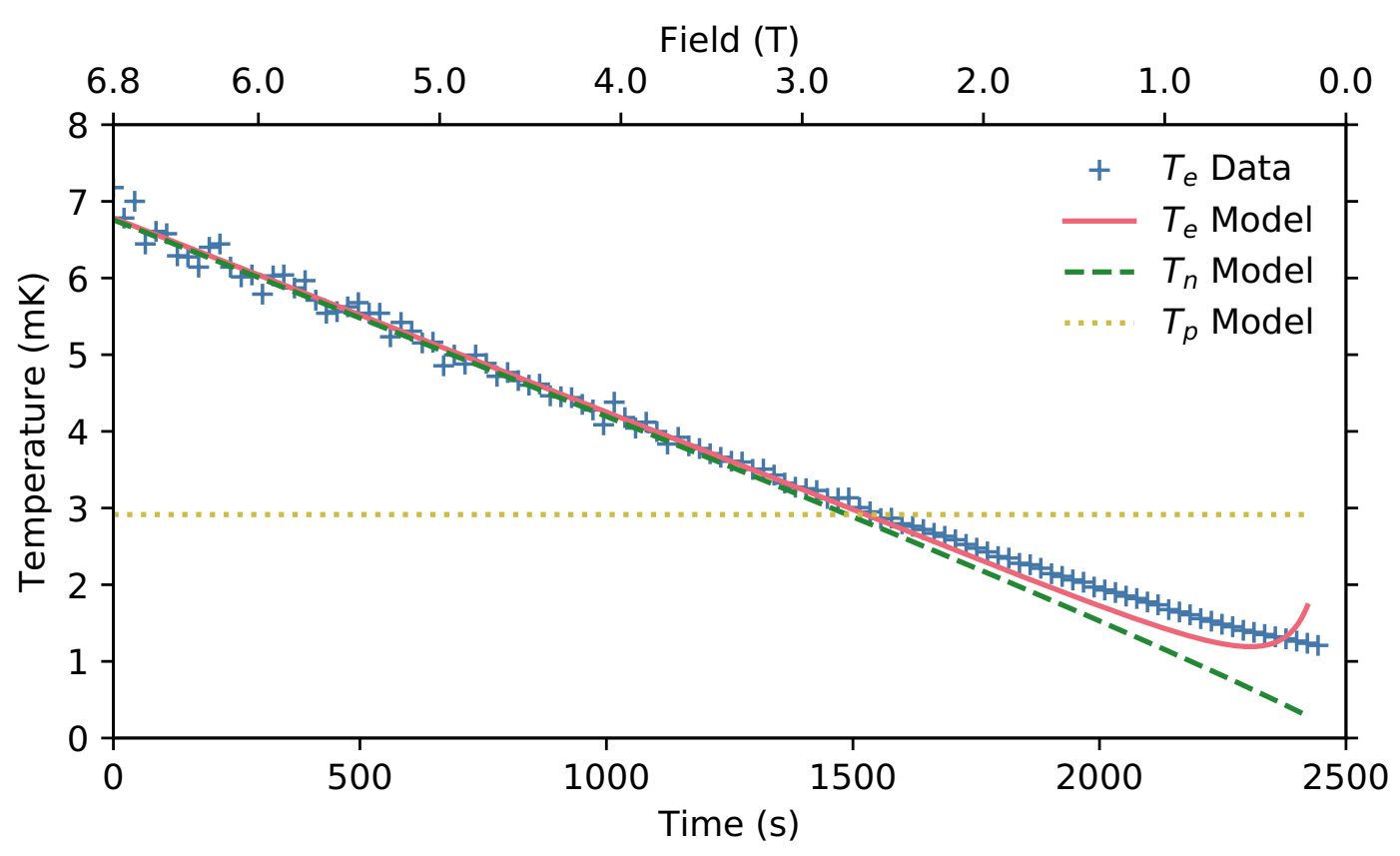

Figure 4.10: Thermal Model Fitted to a $2.7 \mathrm{mT} \mathrm{s}^{-1}$ Demagnetisation. The modelled electron temperatures (solid orange line) are fitted to the measured data (blue pluses). The fitted parameters were $\dot{Q}_{\mathrm{par}}=4.76 \mathrm{fW}$, $V_{\text {eff }}=0.96 \mathrm{~V}$ and $\dot{T}_{p}=0.00 \mu \mathrm{K} \mathrm{s}^{-1}$ (shown by the red dotted line).

tion 3.4.3 was repeated, whereby the sweep rates with the smallest change in $B / T_{e}$ were used in order to limit the entropy change. The unoptimised $2.7 \mathrm{mT} \mathrm{s}^{-1}$ sweep used in the model above, together with the final optimised sweep, are shown by the solid blue and dashed orange lines, respectively, in Figure 4.11. A standard and an optimised sweep from the measurements performed in Chapter 4 are also shown for comparison. We note that the optimised sweep on the wet dilution refrigerator made very little difference to the minimum measured electron temperatures, $1.15 \mathrm{mK}$ and $1.08 \mathrm{mK}$ for the unoptimised and optimised sweeps, respectively. The hold time also saw very little change, with it being $\approx 500 \mathrm{~s}$ at the lowest temperature for both sweeps. This is most likely because the lowest recorded temperature in both cases is inaccurate due to the CBT being far outside its operation range. This is backed up by the electron temperature appearing to saturate around $1.1 \mathrm{mK}$ for both profiles. In any case, however, the temperatures obtained are much lower than those on the dry dilution refrigerator. Given the large uncertainty that results from this issue, the minimum temperature can only be quoted as $(1.1 \pm 0.4) \mathrm{mK}$. 

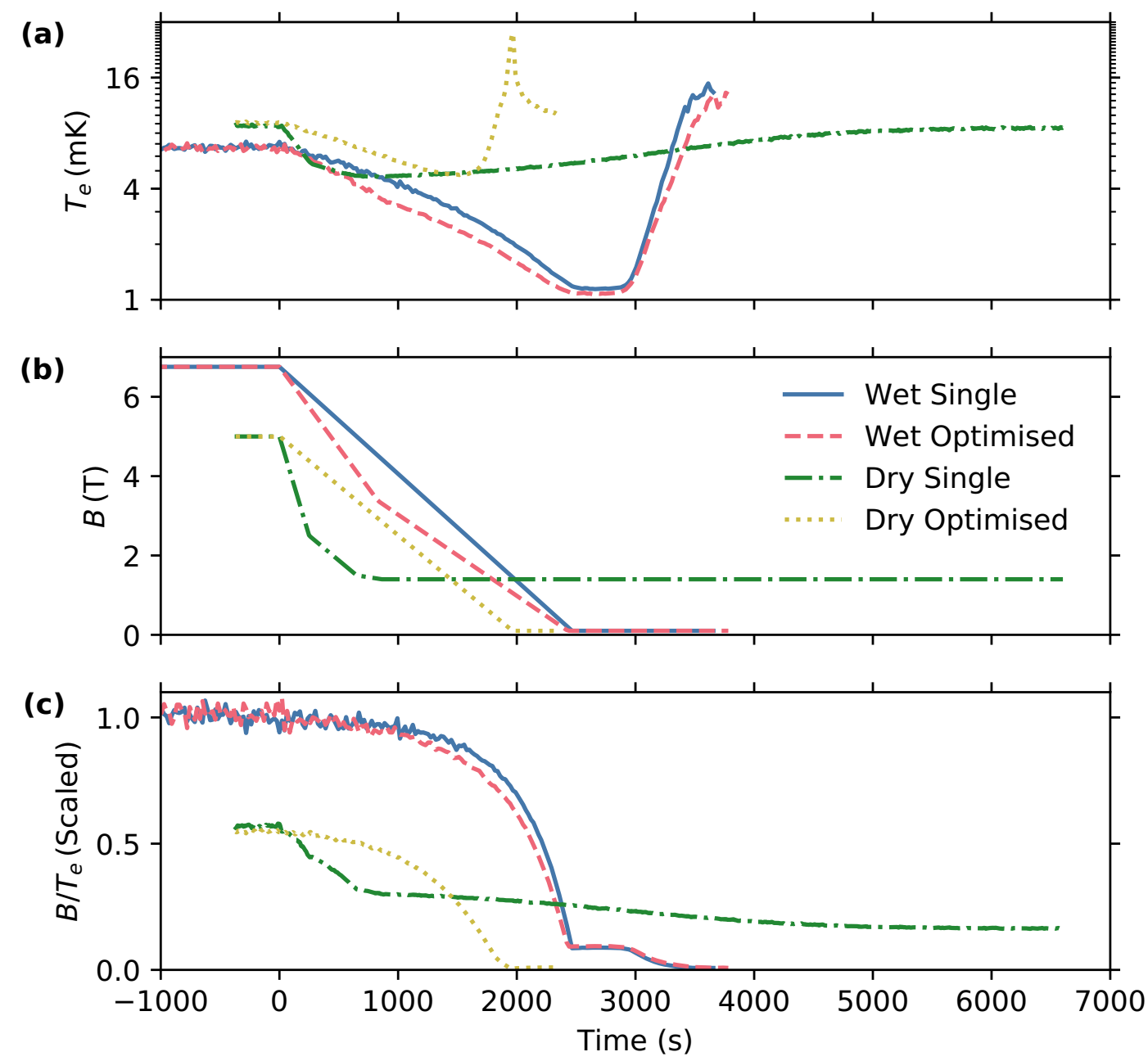

Figure 4.11: Effect of Demagnetisation Profile on CBT Temperature. All parts of this figure are plotted to a common time axis, shown at the bottom of (c). Panel (a) shows how the CBT electron temperature depends on the field sweep profile, shown in (b), for this experiment on a wet dilution refrigerator, and the previous experiment on a dry dilution refrigerator (Chapter 3). Panel (c) shows the deviation from the ideal case of constant entropy $\left(B / T_{e}=\right.$ constant $)$, with the values scaled by the same factor for both the wet and dry cryostats. 


\subsection{Conclusions}

In conclusion, the on-chip nuclear demagnetisation procedure first developed in Chapter 3 was tested on a wet dilution refrigerator and observed to work and produce lower electron temperatures. To measure this a number of issues relating to low temperature CBT measurements had to be accounted for. Some of these, such as the requirement to track the centre of the conductance dip, were successful and will be useful for further low temperature thermometry. However, measuring a CBT at a temperature below its operation range presents an insurmountable problem with the large uncertainties that arise, limiting us to the conclusion that the minimum electron temperature achieved was $(1.1 \pm 0.4) \mathrm{mK}$. Nevertheless, this provides evidence that this cooling technique has the potential to reach record low electron temperatures, and hence motivates the next chapter where this is attempted with a different CBT with a smaller charging energy. 


\section{Chapter 5}

\section{On-Chip Demagnetisation Cooling of a High Capacitance CBT}

The work in this chapter follows from the results shown in Chapter 3, where nuclear demagnetisation of on-chip copper refrigerant was proven to be a viable cooling technique for nanoelectronic devices, and from Chapter 4, where it was shown that using this technique on a wet dilution refrigerator, with a lower base temperature and larger magnetic field, has promise for breaking the $1 \mathrm{mK}$ electron temperature barrier. The electron temperature results in Chapter 4 were, however, inconclusive due to the CBT being used outside the weak Coulomb blockade regime. This chapter describes the attempts at further on-chip cooling using a different CBT which was optimised for operation at much lower temperatures, and hence allowed a minimum electron temperature of $(1.20 \pm 0.03) \mathrm{mK}$ to be recorded. These results were presented in the publication [110], which was drafted by the author of this thesis.

In this chapter, Section 5.1 describes the design and fabrication of the new CBT in addition to the changes necessary for its mounting in a different dilution refrigerator. The characterisation of this new CBT is presented in Section 5.2. The results from applying nuclear demagnetisation cooling are given in Section 5.3, and the effects of heat leaks and the form of the copper on the CBT are then discussed in Sections 5.4 and 5.5, respectively. Finally, the conclusions are presented in Section 5.6. 


\section{$5.1 \quad$ Experimental Set-Up}

The overall experimental set-up for this experiment is very similar to that described in Section 4.1. There are, however, two main differences. The first is, of course, the change in CBT design, made in order to achieve a smaller charging energy to enable lower temperature thermometry. The fabrication of this new device is described in Subsection 5.1.1. Secondly, due to a change in the availability of the dilution refrigerators in the Ultra-Low Temperature Laboratory, a different dilution refrigerator was used for the precooling of the CBT. This necessitated a small number of changes to the cold finger used to mount and heatsink the device to the cryostat. These changes are described in Subsection 5.1.2.

\subsubsection{New CBT Fabrication}

As described in Subsection 2.2.3, the practical operation range of a CBT is given by $0.2<k_{B} T / E_{c}<20$ which, for the previous design of device with $E_{c}=820 \mathrm{neV}$, gives a temperature range $1.9 \mathrm{mK}<T_{e}<190 \mathrm{mK}$. This upper limit is unnecessarily high for a device being used for proof of concept testing a technique aiming to reach the lowest electron temperatures (around $\sim 1 \mathrm{mK}$ ), and, as was found in Chapter 4, the lower temperature limit is too high. There is, however, scope for achieving a more useful temperature range if the charging energy is reduced by a factor $\approx 10$, giving an approximate operation range $200 \mu \mathrm{K}<T_{e}<20 \mathrm{mK}$. The lower limit here is cold enough to accurately report the demagnetisation cooling which, from the work in Chapter 4 , we expect to be down to $\approx 1 \mathrm{mK}$.

It is equally important, however, that the upper limit is not too low, since four conductance curves must be measured at different temperatures in order to selfcalibrate the sensor and enable the use of the much faster pseudo-secondary mode for thermometry during the demagnetisations. Attempting to measure a full conductance curve with a demagnetised sensor, in order to reach a lower temperature, is impractical due to the relatively short cold time available (shorter still with the added Joule heating arising from the DC bias applied during these measurements), and is liable to produce inaccurate results since the temperature will be continuously changing as the conductance curve is measured. Therefore, the minimum temperature usable for the self-calibration is the lowest electron temperature reached when the dilution refrigerator is at its base temperature. The weak electron-phonon coupling means that this temperature is $\approx 4 \mathrm{mK}$, as seen in the lowest temperature conductance curve recorded in Subsection 4.2.2 and as seen in a dedicated study $[49,127]$ for a non-immersed CBT. Given this minimum equilibrium temperature, we see that the maximum CBT temperature must be 
reasonably high in order to allow a reasonable spread of temperatures to be used for the conductance curves. The maximum temperature of $20 \mathrm{mK}$ allows this.

To reduce the charging energy by a factor of 10, the total capacitance per island, $C_{\Sigma}$, must be increased by a factor of 10 , since the charging energy is given by

$$
E_{c} \equiv \frac{N-1}{N} \frac{e^{2}}{C_{\Sigma}}
$$

for $N=33$ the number of tunnel junctions in each series chain (see Subsection 2.2.2). This capacitance is the sum of the capacitances of the two tunnel junctions connected to the island and the stray capacitance of the island to ground. The magnitude of these capacitances can be estimated by assuming they are in the form of a parallel-plate capacitor which has capacitance

$$
C=\frac{\epsilon \epsilon_{0} A}{d}
$$

for $\epsilon_{0}$ the permittivity of free space, $\epsilon$ the relative permittivity and $A$ the cross sectional area of the dielectric material, and $d$ the separation of the metal electrodes. The tunnel junctions in the ex-situ fabricated CBTs are of circular cross section when viewed from above (see Figures 3.1c and 3.2 in Section 3.1), with diameter $0.8 \mu \mathrm{m}$ and approximate thickness $2 \mathrm{~nm}$, as determined from a transmission electron microscope (TEM) image of the junction [89]. The dielectric constant of the $\mathrm{AlO}_{\mathrm{x}}$ tunnel barrier is usually in the range 8-9 [129, 130], although it can vary at high temperature [131] or, as a consequence of the fabrication process, can be reduced to as low as 0.5 [132]. Assuming a dielectric constant of 9 , the capacitance of each junction in the previous CBT is $\approx 20 \mathrm{fF}$, meaning the bulk of the contribution to $C_{\Sigma} \approx 200 \mathrm{fF}$ is from the stray capacitance. Attempting to increase $C_{\Sigma}$ to $\approx 2000 \mathrm{fF}$ by enlarging the junctions requires the development of a new fabrication process able to produce junctions with a much larger area or smaller thickness. Again, developing such a process, which has limited application outside of this project, is undesirable.

Instead, it was decided to increase the stray capacitance of the CBT islands. This was done by conformally coating a pre-existing device with a $\approx 400 \mathrm{~nm}$ thick proprietary dielectric material, deposited by atomic layer deposition (ALD), followed by a metallic layer, as shown in Figure 5.1a. When the device was installed in the same silver package as used before, and the measurement lines were connected by wire bonding, the top metallic layer was also bonded to ground. This creates a parallel plate capacitor between the islands and ground, hence increasing the stray capacitance. The CBT used was to the same design as the previous ones, except it 

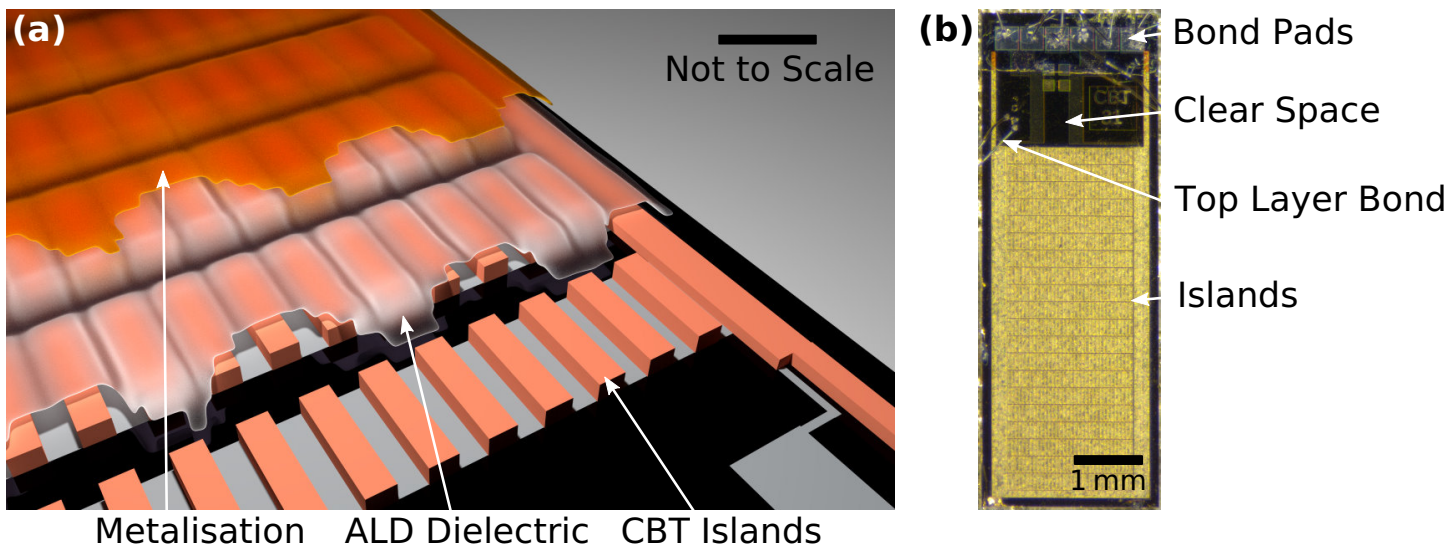

Figure 5.1: Additional Stray Capacitance Added to a CBT. Panel (a) shows a rendering of the extra dielectric and metallic layers added on top a preexisting $\mathrm{CBT}$ in order to increase the stray capacitance and hence reduce the charging energy for lower temperature operation. Panel (b) is a photograph of the chip showing the coating. Note the original copper colour at the top of the chip on the thin metallic leads on the far left and right. Panel (a) is redrawn from [110].

does not feature an on-chip filter. The absence of this filter may have implications for the thermalisation of the sensor and for the noise levels, however, it was unknown if the device would be destroyed during the processing necessary to add this extra capacitance layer, hence it was decided to attempt it on some less precious earlier samples which lack this filter. Additionally, when wire bonding to the top metallic layer, there is a risk of punching through to the lower layers and causing a short circuit. For the devices with filters, the entirety of the $2.3 \mathrm{~mm} \times 6.5 \mathrm{~mm}$ chip is covered with circuitry, meaning that there are no safe locations for wire bonding the top layer where the risk of a short circuit is reduced. However, the devices without filters have $\mathrm{a} \approx 2 \mathrm{~mm} \times 1 \mathrm{~mm}$ empty area above the islands where the filter would be, giving a suitable area for bonding the top layer (see Figure 5.1b). The capacitance created between each island, of cross sectional area $39 \mu \mathrm{m} \times 206 \mu \mathrm{m}$, and the metallic layer, $400 \mathrm{~nm}$ away though a dielectric with $\epsilon \approx 10$, can be estimated using the parallel plate model as $C_{s} \approx 1.8 \mathrm{pF}$. This should lead to a total capacitance per island for this CBT of $C_{\Sigma} \approx 2.0 \mathrm{pF}$, after accounting for the original $200 \mathrm{fF}$, and therefore a charging energy $\approx 90 \mathrm{neV}$, which is $10 \%$ of that for the previous CBT design.

\subsubsection{Precooling}

It was originally intended to cool the new sensor, and test nuclear demagnetisation cooling on it, in Fridge 6 as in Chapter 4. This machine had since become unavailable, however, so it was decided to use a different wet dilution refrigerator labelled 'Fridge 4'. The construction of this fridge was much simpler and followed a more 
straightforward approach to minimising heat leaks than the 'throw the kitchen sink at it' philosophy used on Fridges 5 and 6 . Nevertheless, it does still feature a surrounding Faraday cage, air spring vibration isolation, and was designed for cooling (small) nuclear demagnetisation stages using an $8 \mathrm{~T}$ superconducting solenoid. The base temperature of this dilution refrigerator is $2.3 \mathrm{mK}$ [133], and is the machine used for the immersion and vacuum cooling of a CBT in [49]. In that study, the CBT with gold thermalisation blocks on each island was cooled in vacuum to $4.0 \mathrm{mK}$ via silver sinters immersed in the MXC. The weaker electron-phonon coupling in the copper thermalisation blocks (copper $\Sigma=2.0 \mathrm{GW} / \mathrm{m}^{3} \mathrm{~K}^{5}$ [106], gold $\Sigma=2.4 \mathrm{GW} / \mathrm{m}^{3} \mathrm{~K}^{5}$ [54]) on the CBT in use for Chapter 4 meant that the lowest equilibrium temperature obtained was $4.3 \mathrm{mK}$, despite the lower MXC temperature. As such, despite the higher base MXC temperature, Fridge 4 is also sufficient for precooling the CBT for on-chip nuclear demagnetisation.

Fridge 4 also features a mixing chamber with a demountable base, allowing access to the helium mixture. It is however much smaller than the mixing chamber of Fridge 6, and the centre of the magnetic field applied by the solenoid is at a different height. As such, a replacement cold finger had to be built which was compatible with this dilution refrigerator and which located the CBT at the centre of the magnetic field. This cold finger is to the same general design as the one shown in Figure 4.2 of Chapter 4, but with different dimensions. It has a silver sinter connected to the CBT package and four other silver sinters connected to the four measurement leads via shielded silver wires, as shown in Figure 5.2. Other than dimensions, the only tangible difference between this cold finger and the one in Chapter 4 is the lack of a diagnostic post, since Fridge 4 has a resistive heater, tuning fork and VWR integrated into the MXC.

\subsection{CBT Characterisation}

Four conductance curves were measured at four different temperatures, starting with the base temperature curve at $4.3 \mathrm{mK}$ and then increasing in $\approx 5 \mathrm{mK}$ intervals, as shown in Figure 5.3. Note that the base temperature curve is at the same temperature as in Chapter 4, indicating that the thermalisation of the sensor is as good in Fridge 4 as it was in Fridge 6. These measurements were made in two halves, from zero bias outwards, to enable the full tunnelling model to account for the Joule heating of the sensor, as described in Subsection 4.2.1.

The measurements were made using the same electrical set-up as described in Subsection 3.2.2, with the exceptions of the missing on-chip filter and a different current source, which was replaced with one with different gains more suited to 


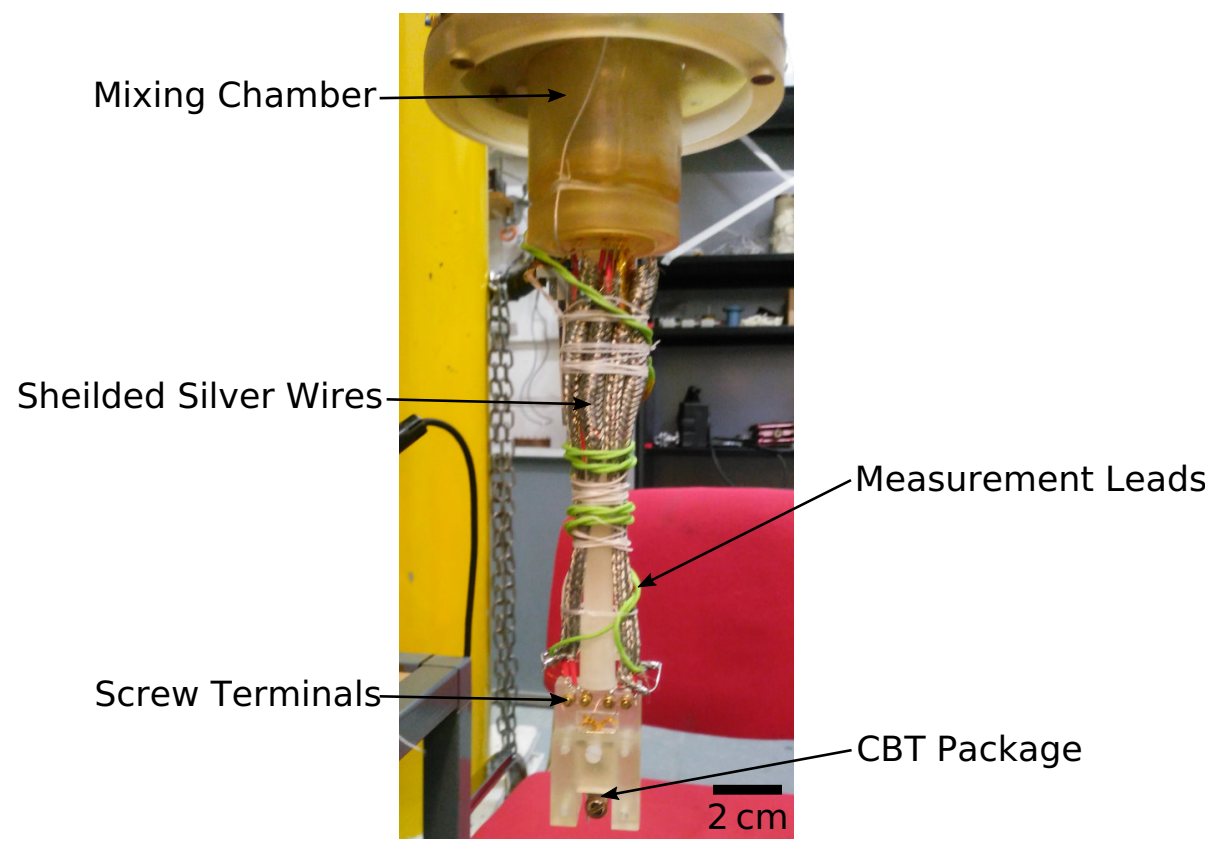

Figure 5.2: Cold Finger Used on Fridge 4. This was built to the same general design as the previous cold finger shown in Figure 4.2, but had different dimensions, as required for Fridge 4.

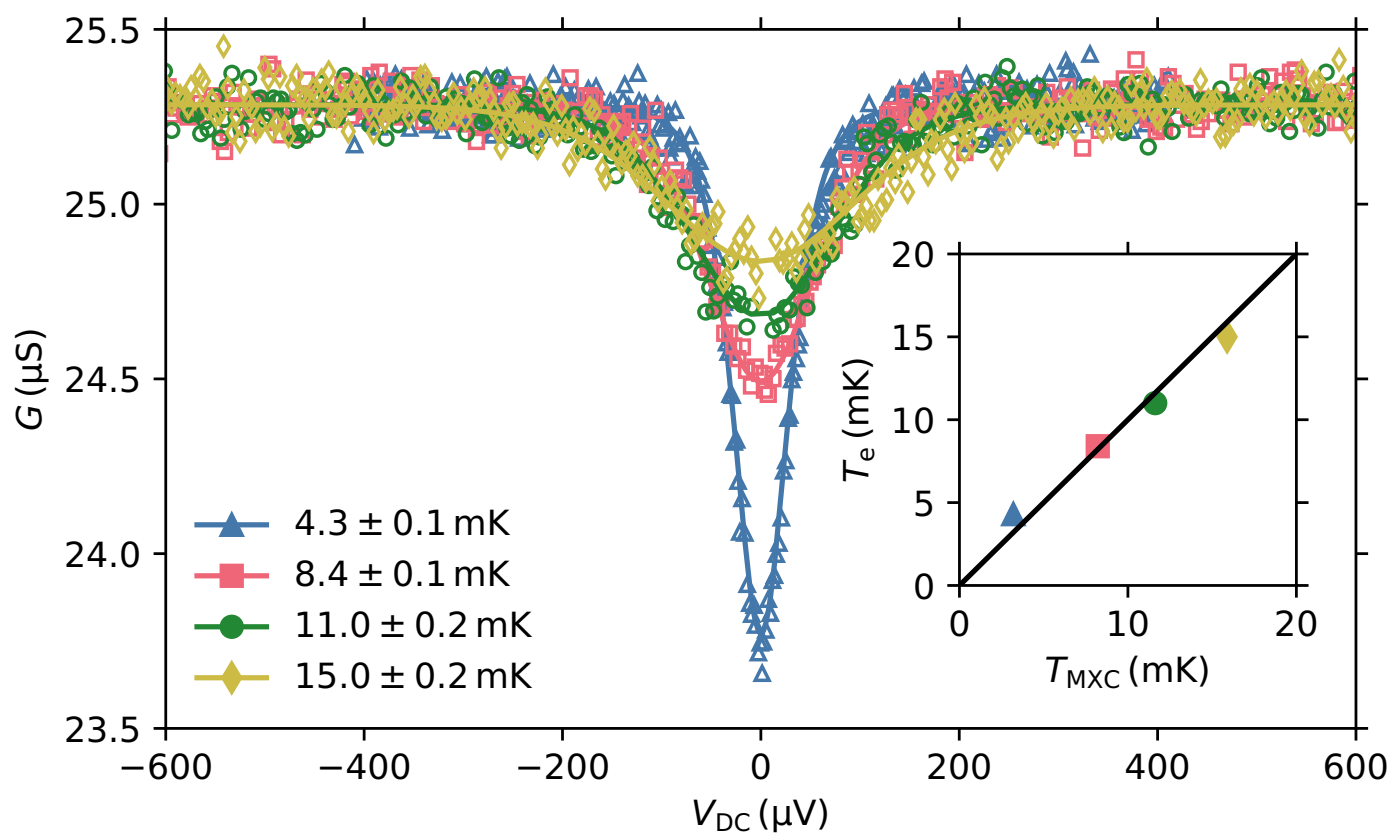

Figure 5.3: High Capacitance CBT Conductance Curves. The symbols show the measured values and the lines show the fits to a full tunnelling model, from which the temperatures shown in the legend were extracted. The inset shows the extracted electron temperatures against the temperatures the mixing chamber was heated to. Note the shallower depth of the conductance dip, as reflected by the smaller y-axis range, compared to the CBT data shown in Figures 3.6, 3.7 and 4.6. The solid line in the inset is $T_{e}=T_{\mathrm{MXC}}$ for reference. This data was measured at a field of $100 \mathrm{mT}$. This figure is redrawn from [110]. 
the smaller excitation current used here $(10 \mathrm{pA}$ at $13 \mathrm{~Hz})$ and smaller DC biases needed for the narrower curves at the lower temperatures at which self-calibration is performed. This current source has the gains $A_{\mathrm{ac}}=100 \mathrm{pA} \mathrm{V}^{-1}$ and $A_{\mathrm{dc}}=$ $10 \mathrm{nA} \mathrm{V}^{-1}$.

The sensor parameters extracted from the fit to the three warmest conductance curves were $C_{\Sigma}=(1.155 \pm 0.008) \mathrm{pF}$ and $R_{T}=(23.9698 \pm 0.0008) \mathrm{k} \Omega$. The value of $R_{T}$ is similar to those found for the sensors used in Chapters 3 and 4 , as expected given the underlying tunnel junction device is of the same design in all three cases. This does, however, give an indication that no damage occurred to the sensor, such as electrically shorted islands, during the processing used to add the additional stray capacitance. The value of $C_{\Sigma}$ clearly has been significantly increased from the original value of $C_{\Sigma} \approx 200 \mathrm{fF}$, however it is approximately half as much as estimated earlier $(\approx 2 \mathrm{pF})$, possibly because the dielectric constant of the proprietary insulating layer was not as high as expected.

This value of $C_{\Sigma}$ gives a charging energy $E_{c}=(134.5 \pm 0.9) \mathrm{neV}$. This results an operation range, assuming the background charge distribution is simulated (see Subsection 2.2.3), of

$$
310 \mu \mathrm{K}<\frac{E_{c}}{k_{B} T}<31 \mathrm{mK}
$$

giving an uncertainty of $2 \%$ at the lowest temperatures as a result of the range of possible background charge distributions. This is sufficiently low for the temperatures expected during the demagnetisations. It also, at the time of writing and to the best of our knowledge, gives the lowest reported CBT operation temperature at $2 \%$ uncertainty, c.f. $1.9 \mathrm{mK}$ for the device used in Chapters 3 and $4,1.3 \mathrm{mK}$ by Palma et. al. [134] and $540 \mu \mathrm{K}$ by Sarsby et. al. [50]. This lower operation range is reflected in Figure 5.4, which graphically shows the self-calibration lookup data for this CBT. Note that the zero-bias conductances at a given temperature are much higher than is seen for the previous CBTs in Figures 3.8 and 4.8. Additionally, the curve rapidly steepens around $20 \mathrm{mK}$, representing the loss of temperature sensitivity above this, where the conductance vs. bias voltage characteristic is essentially a straight horizontal line.

\subsection{Demagnetisation Cooling}

Prior to demagnetisation, the solenoid was ramped up to full field $(8.0 \mathrm{~T})$ and then the CBT electrons were allowed to thermalise. Similar to in Chapter 4, thermal equilibrium at $6 \mathrm{mK}$ would be reached after $\approx 80 \mathrm{~h}$, although precooling overnight resulted in an acceptable starting temperature of $8 \mathrm{mK}$. The magnetic field was 


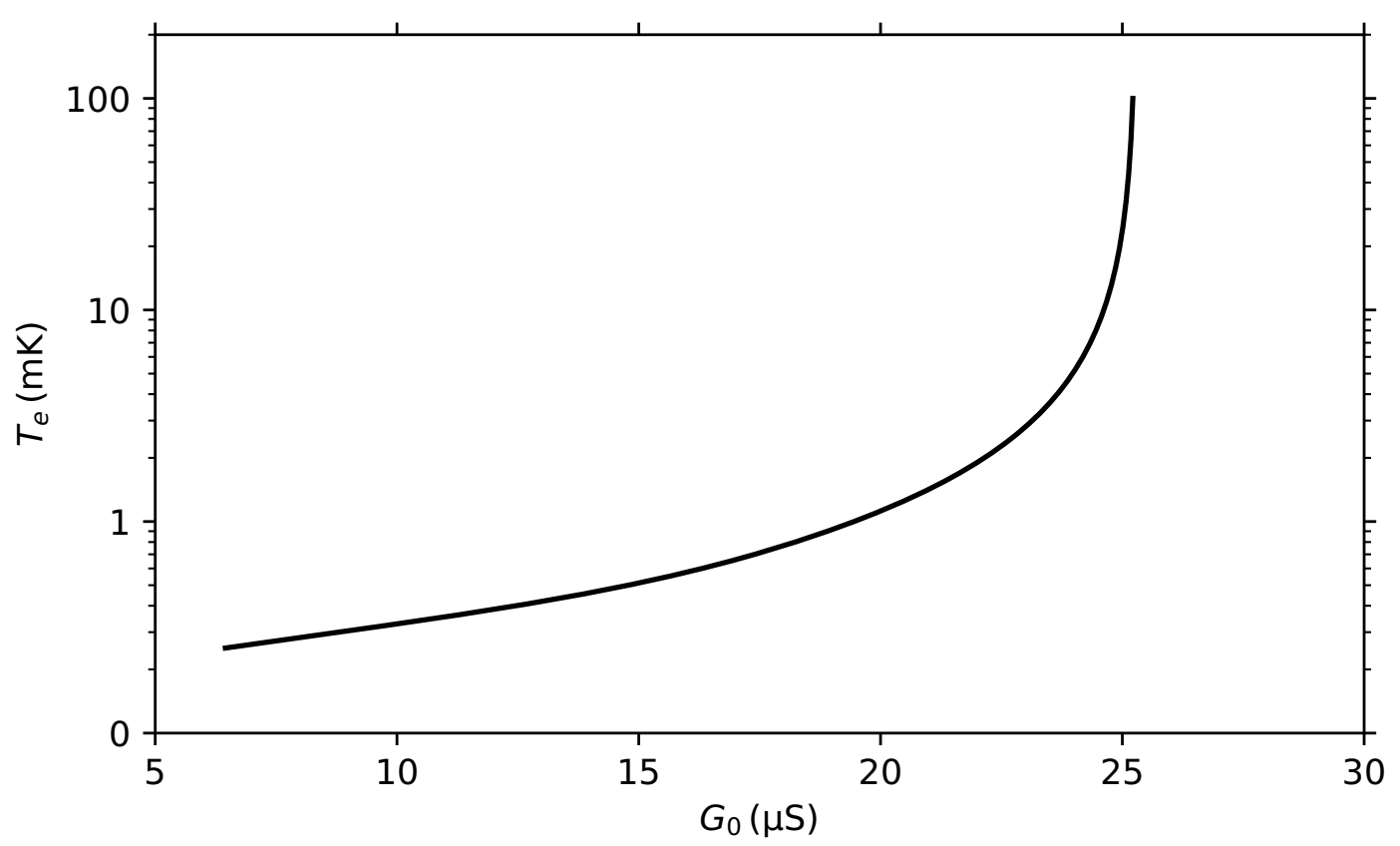

Figure 5.4: CBT Temperature vs. Conductance Self-Calibration Curve. The data in this plot is used to calculate the CBT's electron temperature $T_{e}$ from a measurement of the zero bias conductance $G_{0}$.

then swept down using a selected ramp rate profile while recording the zero bias CBT conductance every 20 seconds and simultaneously using the signal at twice the excitation frequency to track the location of the conductance minimum.

The magnetic field was swept down between $8.0 \mathrm{~T}$ and $0.1 \mathrm{~T}$ at several different rates, and then the results compared to enable optimisation. An example sweep at $3.0 \mathrm{mT} \mathrm{s}^{-1}$ is shown in Figure 5.5. The time dependence of the resulting electron and MXC temperatures is similar to those seen before, except that the MXC temperature reaches a higher value of $20 \mathrm{mK}$ and, following the demagnetisation, this temperature continues to rise up to $\approx 30 \mathrm{mK}$ (not shown in the figure). This demagnetisation was fitted to the thermal model described in Subsection 3.4.2, as shown in Figure 5.6. This fit, while not perfect at the lowest temperatures, more accurately reproduces the electron temperature during the demagnetisation than the attempted fit to the data taken using the low capacitance CBT (Chapter 4, Figure 4.10), indicating that the origin of the poor fit and unrealistic parameters earlier was the result of inaccurate thermometry. The fit parameters here are similar to those measured in the dry dilution refrigerator (Chapter 3, Figure 3.14), with both demagnetisations featuring $\dot{Q}_{\mathrm{par}} \approx 6 \mathrm{fW}$, the value of $\dot{T}_{p}$ here being roughly double that found in Chapter 3, and the effective volume for the electron phonon coupling $V_{\text {eff }}$ being $7.7 \%$ and $12 \%$ of the true volume here and in Chapter 3, respectively. 


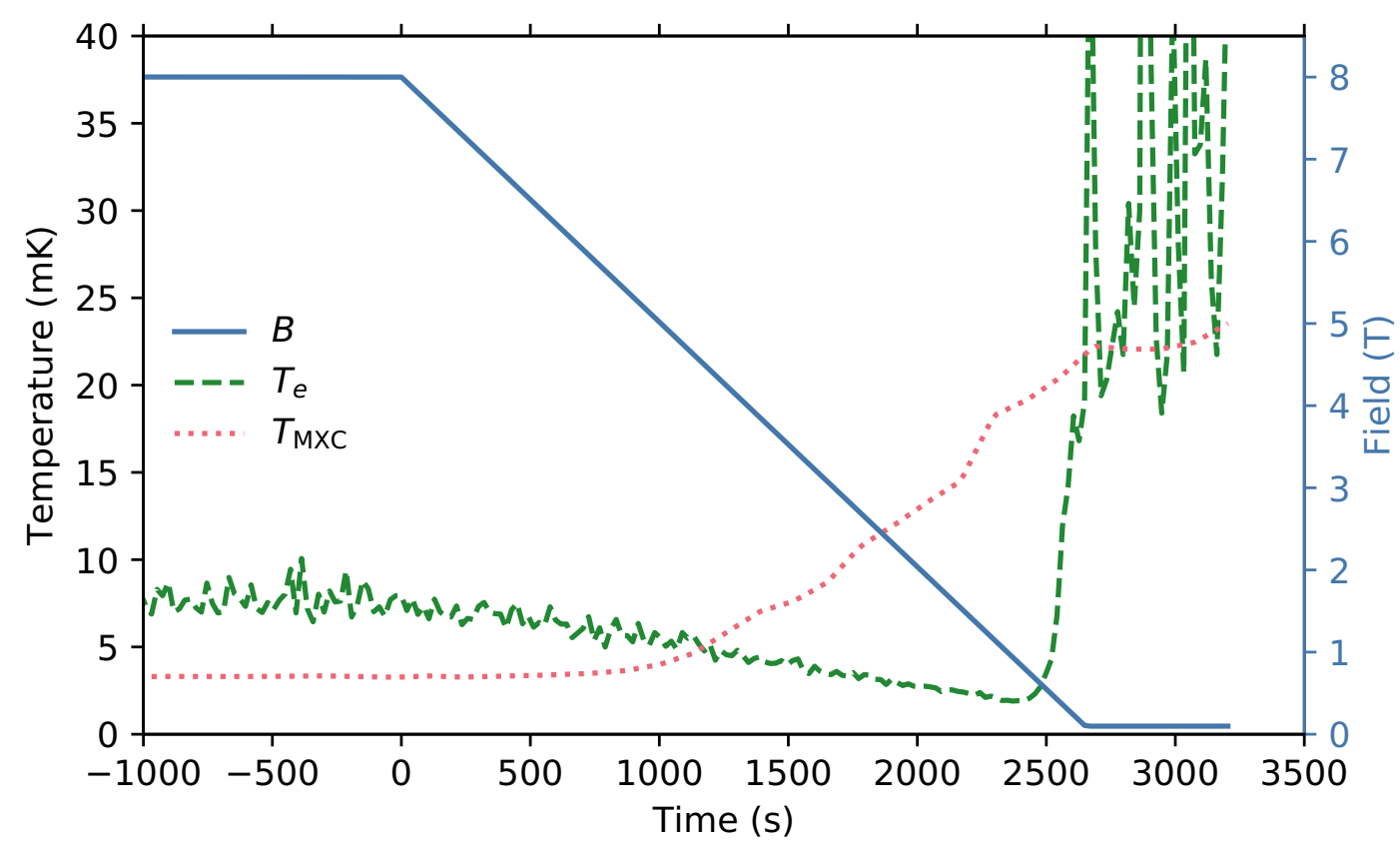

Figure 5.5: CBT Electron Temperature During Demagnetisation from 8.0 T to $0.1 \mathrm{~T}$ at $3.0 \mathrm{mT} \mathrm{s}^{-1}$. The very noisy $T_{e}$ values near $3000 \mathrm{~s}$ are the result of the poor temperature sensitivity of this CBT above $\approx 20 \mathrm{mK}$, i.e. a small conductance change here corresponds to a large temperature change (see Figure 5.4), so the small amount of noise in $G_{0}$ creates a large amount of noise in $T_{e}$.

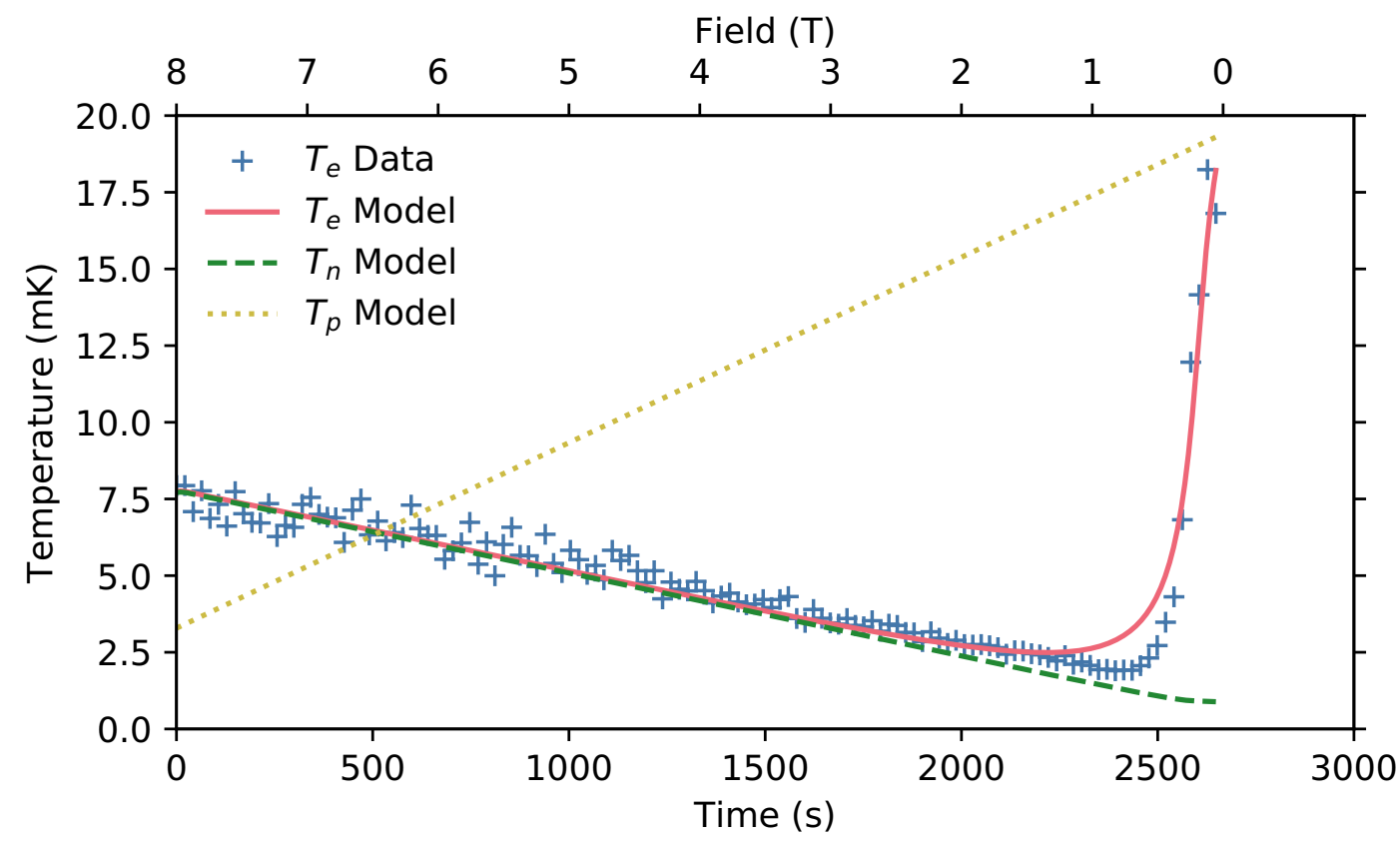

Figure 5.6: Thermal Model Fitted to a $3.0 \mathrm{mT} \mathrm{s}^{-1}$ Demagnetisation. The modelled electron temperatures (solid red line) are fitted to the measured data (blue pluses). The fitted parameters were $\dot{Q}_{\mathrm{par}}=6.01 \mathrm{fW}, \dot{T}_{p}=6.11 \mu \mathrm{K} \mathrm{s}^{-1}$ (shown by the dotted yellow line) and $V_{\text {eff }}=0.077 \mathrm{~V}$. The nuclear spin temperature is shown by the dashed green line. 


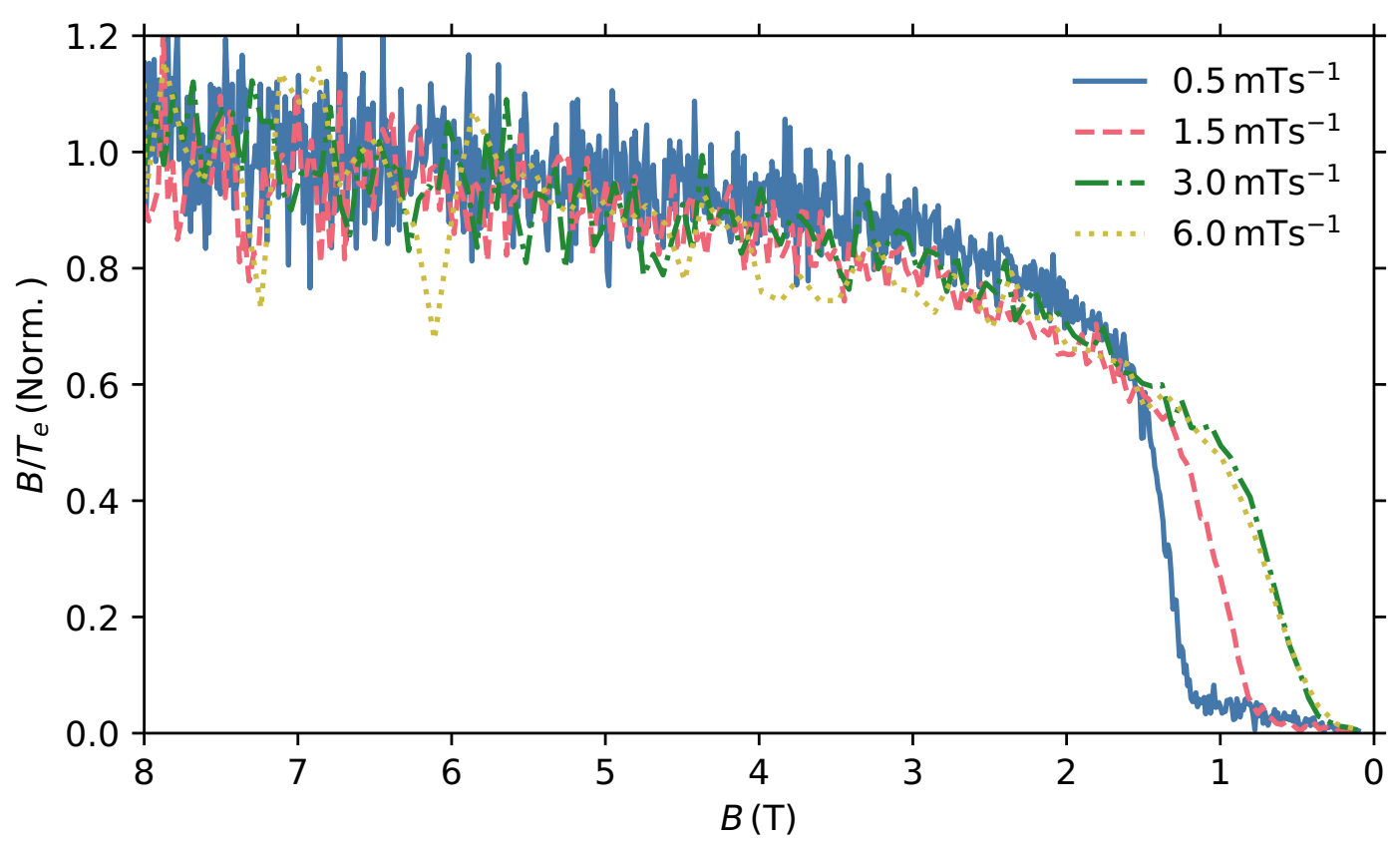

Figure 5.7: Evolution of $B / T_{e}$ Used for Demagnetisation Optimisation. This shows the amount of deviation from the ideal adiabatic case of constant $B / T$ for four different magnetic field sweep rates. Each curve is normalised to 1 at the start of the sweep.

Figure 5.7 shows how the variation of $B / T_{e}$ during a demagnetisation depends on the sweep rates used. In contrast to what was seen in Figure 3.15 in Chapter 3, there is very little variation between the sweep rates for fields above $\approx 1.8 \mathrm{~T}$, although any difference present may be masked by the noisier signal. More surprising is that towards the end of the demagnetisation, the rate of entropy change is larger for the slower rates, the opposite of what was observed last time, and the opposite to what would be expected if this entropy change is dominated by eddy current heating (the power from which goes as $\dot{B}^{2}[25]$ ). This suggests that there is a large heat leak occurring below $1.8 \mathrm{~T}$ which is independent of the ramp rate, and hence that it is important to demagnetise sufficiently quickly that the heat flow to the nuclear spins, $\dot{Q}_{\text {en }}$, is able to compensate for it. There is, however, little difference between the $3.0 \mathrm{mT} \mathrm{s}^{-1}$ and $6.0 \mathrm{mT} \mathrm{s}^{-1}$ sweeps at the end, suggesting that at this point the eddy current heating is once again becoming relevant.

For the optimisation of the magnetic field sweep rate above $1.8 \mathrm{~T}$, the empirical approach used previously is impossible since the noise in the data in Figure 5.7 masks any useful information. As an alternative, a full thermodynamic model of the nuclear cooling process could be used to calculate the ideal demagnetisation rate $[135,136]$, which can then be approximated by using a linear rate profile, $\dot{B} \propto B$, as used in [50] to keep $\dot{B} / B$ constant. Unfortunately, the power supply 
for the magnet used in Fridge 4 is not capable of continuous rate adjustment, which ruled out these approaches. Instead, we approximate the linear rate profile by halving the sweep rate when the magnetic field has completed half of its total sweep (e.g. for a demagnetisation from $8.0 \mathrm{~T}$ to $1.8 \mathrm{~T}$, the rate would be halved at $4.9 \mathrm{~T}$ ). The difference made by this part of the optimisation must be small, given Figure 5.7, as such going beyond this approximation is likely unnecessary.

Below $1.8 \mathrm{~T}$, the demagnetisation was optimised in two different ways. One approach was to stop the demagnetisation at $1.8 \mathrm{~T}$. This avoids the large heat leak that occurs below this field (see Section 5.4 for a discussion on the origins of this), and results in the nuclear spin heat capacity remaining relatively large, hence providing a long hold time, at the expense of a higher theoretical minimum temperature. The other approach was to keep reducing the field at the optimal rate of $3.0 \mathrm{~m} \mathrm{~T} \mathrm{~s}^{-1}$ below $1.8 \mathrm{~T}$ and then stop the sweep as soon as the electrons begin to heat. This latter approach will optimise the sweep for the lowest electron temperatures, at the expense of hold time.

Figure 5.8 shows the temperatures (Figure 5.8a) that result from these two optimised magnetic field sweeps (Figure 5.8b). For comparison it also shows the optimal demagnetisation profile from the dry cryostat in Chapter 3. The profile optimised for the minimum electron temperature reaches $T_{e}=(1.20 \pm 0.03) \mathrm{mK}$, held below $1.3 \mathrm{mK}$ for $1500 \mathrm{~s}$. To the best of our knowledge, this is the lowest electron temperature inside a nanoelectronic device achieved using only on-chip magnetic refrigerant. This low temperature is then followed by the rapid warming of the electrons to above $\approx 30 \mathrm{mK}$, the highest temperature reliably measurable with this CBT, which coincides with a very fast change in entropy, as seen in Figure 5.8c. The sweep optimised for a longer hold time stops the magnetic field ramp at $1.8 \mathrm{~T}$, giving a minimum electron temperature of $(1.58 \pm 0.04) \mathrm{mK}$, held below $1.7 \mathrm{mK}$ for $2300 \mathrm{~s}$. The electron temperature then gradually increases back to the base temperature of the dilution refrigerator over $\approx 4 \mathrm{~h}$ (not shown).

\subsection{Heat Leaks}

As stated before, the thermal model includes a parasitic heat leak into the electrons, $\dot{Q}_{\text {par }}$, which gives the total heat input into the electron subsystem except for that entering via the electron-phonon coupling. There are, however, two distinct parts to this heat leak: There is the static heat leak, $\dot{Q}_{\text {stat }}$, which is always present and is the result of electronic noise and radiation, and there is the dynamic heat leak, $\dot{Q}_{\text {dyn }}$, which is only present while sweeping the magnetic field, and hence is primarily the result of eddy current heating. The total heat leak is the sum of 

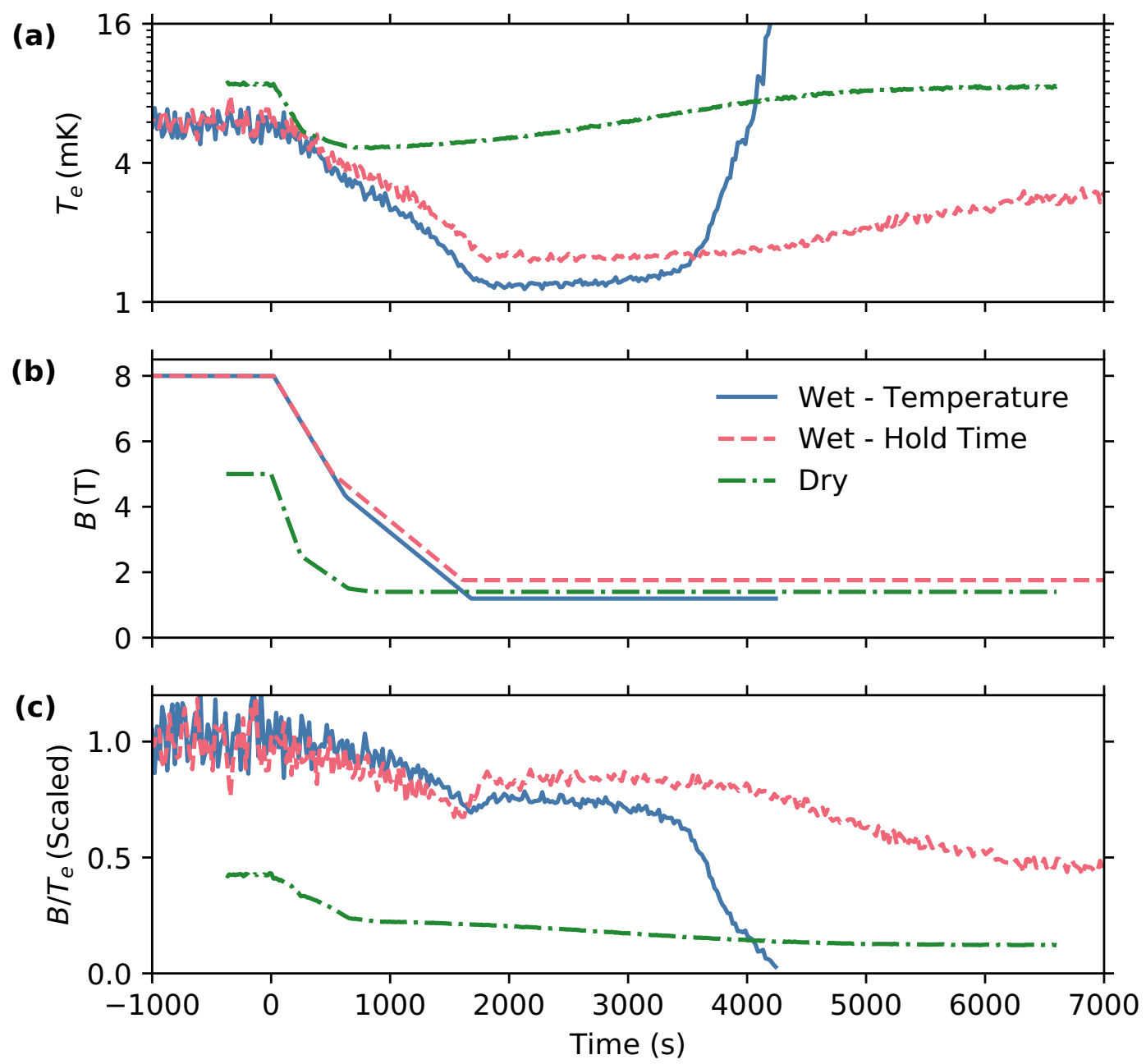

Figure 5.8: Demagnetisation Profiles Summary. All parts of this figure are plotted to a common time axis, shown at the bottom of (c). Panel (a) shows how the CBT electron temperature depends on the field sweep profile, shown in (b). The sweeps in the wet cryostat were optimised for the lowest temperature (solid blue line) and longest hold time (red dashed line), and are shown with the optimised sweep from the dry cryostat (dash-dotted green line) for comparison. Panel (c) shows the deviation from the ideal case of constant entropy $\left(B / T_{e}=\right.$ constant $)$, with the values scaled to a dimensionless number by the same factor for both the wet and dry cryostats. The data in this figure was included in a similar figure in [110] 
these parts, $\dot{Q}_{\mathrm{par}}=\dot{Q}_{\mathrm{stat}}+\dot{Q}_{\mathrm{dyn}}$.

The total heat leak, $\dot{Q}_{\mathrm{par}}$, can be extracted from fits to the thermal model described in Subsection 3.4.2, since this is only fitted to the data recorded while the magnetic field was being swept. This procedure gives the heat leaks shown in Figure 5.9a, which shows a straight line of gradient 2 for the heat leaks on the wet cryostat when plotted on logarithmic axes, suggesting a $\dot{B}^{2}$ dependence. This is as expected, since the heating power, in an object with volume $V$ made of a material with resistivity $\rho$, that results from eddy current heating is of the form [37]

$$
\dot{Q}_{\mathrm{dyn}}=\frac{G V \dot{B}^{2}}{\rho}
$$

for magnetic field sweep rate $\dot{B}$ and a geometry-dependent factor $G$ with dimensions of length-squared. This is in contrast with [103], where a linear dependence in $\dot{B}$ was observed, and with the dry cryostat heat leaks here which, given the curve seen in Figure 5.9a, do not appear to follow any power law.

In Figure $5.9 \mathrm{~b}$, the total heat leak $\dot{Q}_{\text {par }}$ is plotted against $\dot{B}^{2}$ and a linear fit performed. Ideally, for an improved fit, there would be more data points with a better distribution in $\dot{B}^{2}$, however this was not done due to the long time (magnetisation, precool and demagnetisation) each measurement takes and since the main aim was to optimise the process for the lowest temperatures, not measure heat leaks. For a cube with side $a$, the geometric factor $G=a^{2} / 32$ [23] can be used with Equation 5.4 and the gradient of the fit in Figure 5.9b to show that the eddy current heating per island is consistent with it acting over a copper cube with side $a \approx 1 \mathrm{~mm}$. This is considerably larger than the island itself, however, this could reflect some eddy current heating being produced externally and conducted to the island.

In principle, the offset of the fit in Figure $5.9 \mathrm{~b}$ should be the static leak, however here it is negative and small in magnitude. This may simply be because of the uncertainty in the fit, or may reflect the static heat leak being mainly the result of the electron-phonon coupling, so is not included in $\dot{Q}_{\text {par }}$ by the model. Instead, for the two optimised demagnetisation profiles in Figure 5.8, the static heat leaks can be calculated from the data collected after the magnetic field sweep ends. The nuclear heat capacity is given by (Equation 2.14 in Subsection 2.1.2)

$$
C_{n}=\frac{\lambda n\left(B^{2}+b^{2}\right)}{\mu_{0} T_{n}^{2}},
$$

for $\lambda$ the nuclear Curie constant, $\lambda / \mu_{0}=3.22 \mu \mathrm{J} \mathrm{K} \mathrm{T}^{-1} \mathrm{~mol}^{-1}$ for $\mathrm{Cu}, n$ the num- 

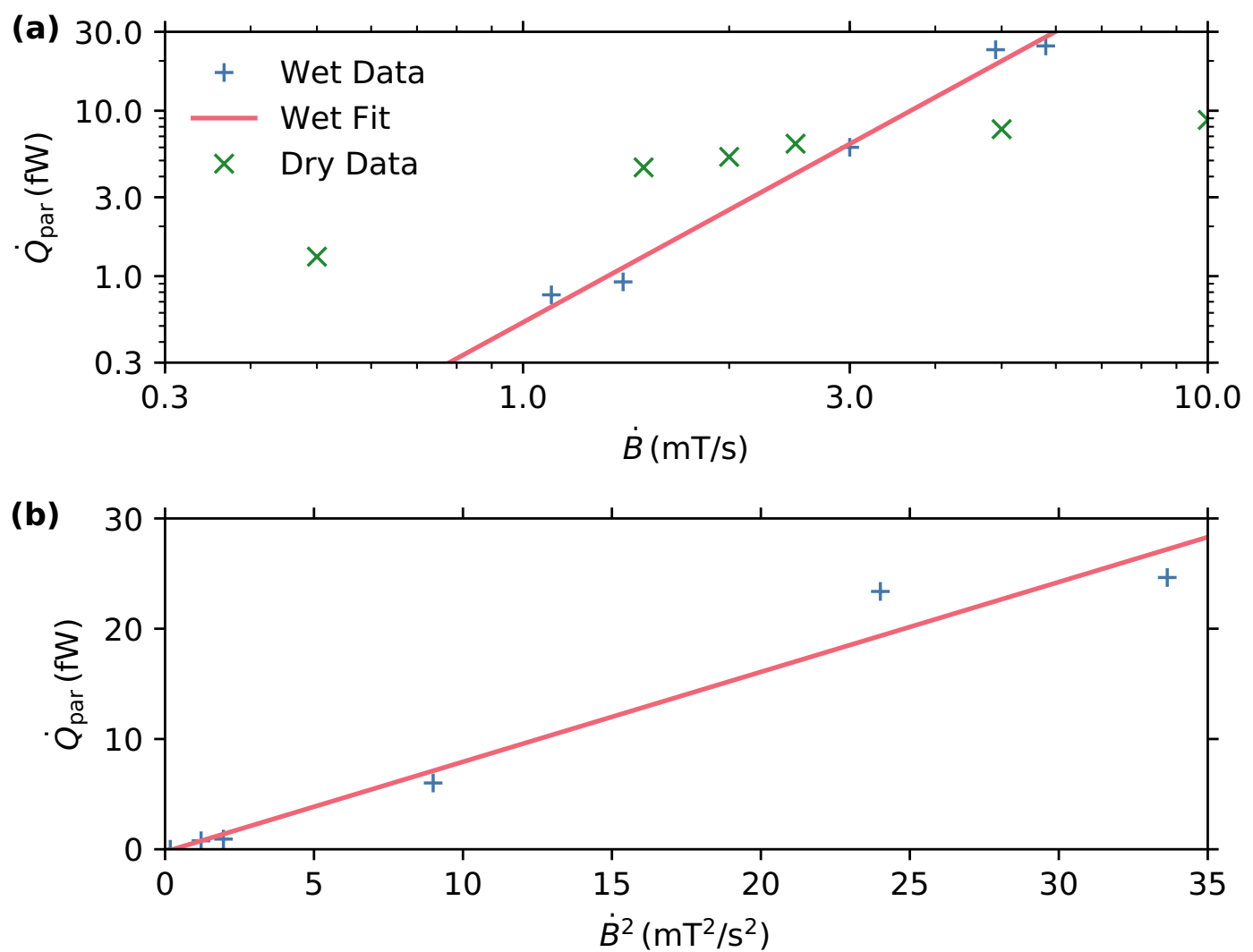

Figure 5.9: Dynamic Heat Leaks with Sweep Rate. Panel (a) shows the total parasitic heat leaks for different demagnetisation rates on the wet (blue pluses) and dry (green crosses) cryostats. The data from the wet cryostat fits the straight red line of gradient 2 on a logarithmic scale, indicating a $\dot{B}^{2}$ dependence. This is verified in Panel (b) where $\dot{Q}_{\text {par }}$ is plotted against $\dot{B}^{2}$. Here, the red line is a linear fit with gradient $(0.82 \pm 0.11) \mathrm{nWs}^{2} / \mathrm{T}^{2}$ and offset $(-0.22 \pm 1.90) \mathrm{fW}$. 


\begin{tabular}{ccccc}
\hline Fridge & Optimisation & $B_{f}(\mathrm{~T})$ & $1 / \dot{T}_{e}\left(\mathrm{~K}^{-1} \mathrm{~s}^{-1}\right)$ & $\dot{Q}_{\mathrm{par}}(\mathrm{fW})$ \\
\hline Wet & Temperature & 1.2 & -0.97 & 31 \\
Wet & Hold Time & 1.8 & -0.016 & 1.2 \\
Dry & - & 1.4 & -0.026 & 1.2 \\
\hline
\end{tabular}

Table 5.1: CBT Heat Leaks at Constant Magnetic Field. These heat leaks were extracted from the fits to the warm up curves shown in Figure 5.10 using Equation 5.6.

ber of moles of copper per island $\left(6.96 \times 10^{-9}\right.$ mol here $), B$ the applied magnetic field and $b=0.36 \mathrm{mT}$ the effective internal dipole field in copper [23]. Following demagnetisation to a final field $B_{f}$, assuming a constant heat leak $\dot{Q}$, we can use Equation 5.5 to find the relation

$$
\frac{\mathrm{d}}{\mathrm{d} t}\left(\frac{1}{T_{n}}\right)=-\dot{Q}\left(\frac{\lambda n\left(B_{f}^{2}+b^{2}\right)}{\mu_{0}}\right)^{-1}
$$

As such, we see that the value of $1 / T_{n}$ should reduce linearly with time at a rate proportional to the static heat leak into the system. Although we do not measure the nuclear spin temperature $T_{n}$ directly in these experiments, the heat capacity of the electrons is sufficiently small that the relatively large heat flow between the electrons and the nuclear spins results in the electrons rapidly thermalising, meaning that the electron temperature is effectively held at the nuclear spin temperature, $T_{n}=T_{e}$. The warming of the CBT following the sweeps shown in Figure 5.8 is shown in Figure 5.10a, and graph of $1 / T_{e}$ against time is shown in Figure 5.10b, which does show a straight line warmup trace after a short period when the CBT begins to warm. Using Equation 5.6, the gradients of these warmup traces give the heat leaks shown in Table 5.1.

The $1.2 \mathrm{fW}$ static heat leak per island, seen for the hold time optimised sweep, is reasonable, although is perhaps disappointing since it was expected that the static heat leak would be smaller on the wet dilution refrigerator than on the dry due to the better electrical shielding and vibration isolation. On the other hand, the heat leak seen in the temperature optimised sweep, $31 \mathrm{fW}$, is surprisingly large, much larger than what would be expected to occur from further eddy current heating between $1.8 \mathrm{~T}$ and $1.2 \mathrm{~T}$. It is therefore indicative of significant excess heating of the dilution refrigerator during this part of the sweep. This heating has been observed before in this dilution refrigerator in a study into the demagnetisation of solid ${ }^{3}$ He in Aerogel [137].

In the Aerogel study, a bulk demagnetisation cell was used in place of the cold finger used here, and it was found that cooling below $\approx 500 \mu \mathrm{K}$ was impossible 

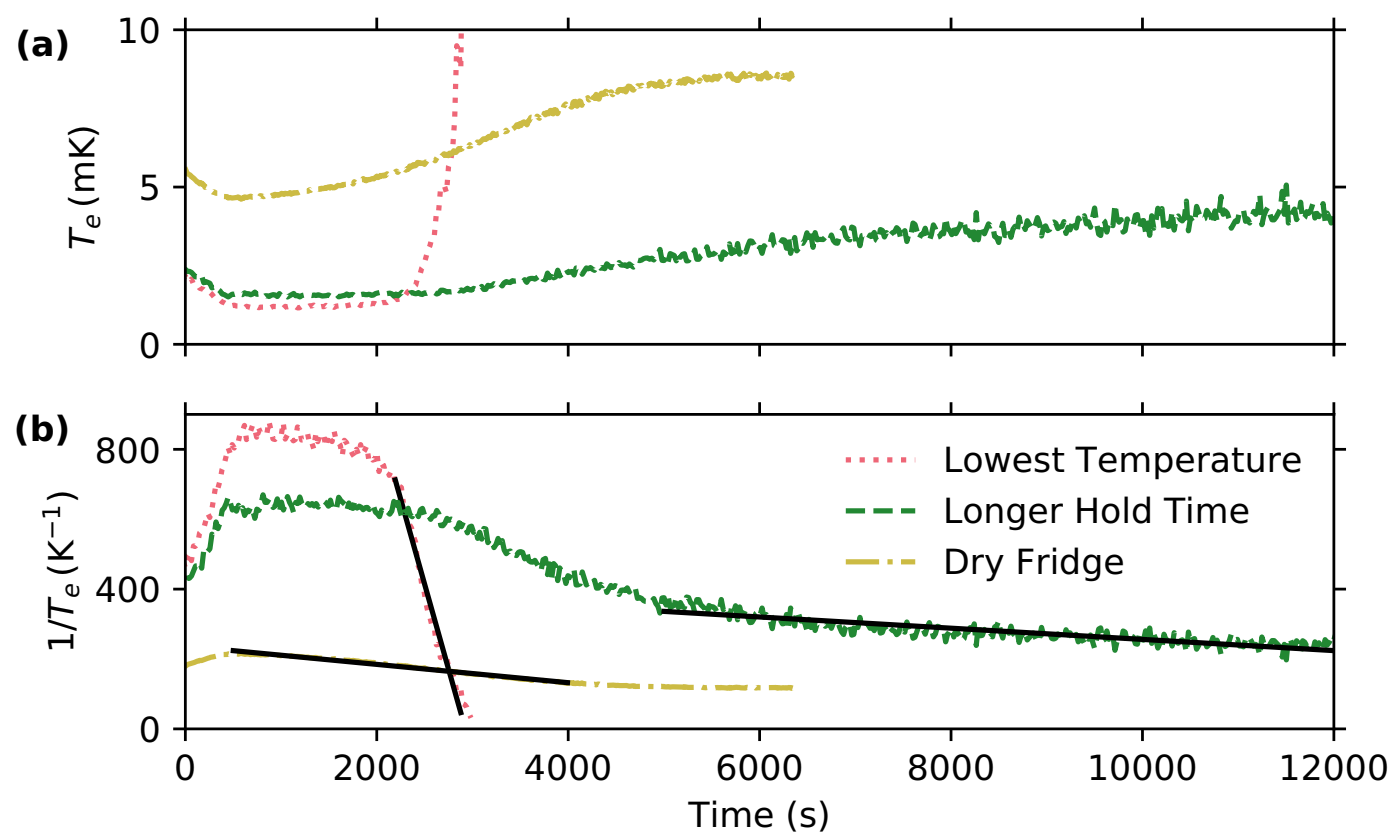

Figure 5.10: Static Heat Leaks. During the warmup following a demagnetisation, shown in panel (a), the rate of change of $1 / T_{e}$ becomes constant, as shown in panel (b), and is fitted with the solid black lines. This is inversely proportional to the static heat leak. This figure shows the optimised sweep profiles on the dry dilution refrigerator and the sweeps optimised for the lowest temperature and a longer hold time on the wet dilution refrigerator. The data in this figure was included in a similar figure in [110]. 


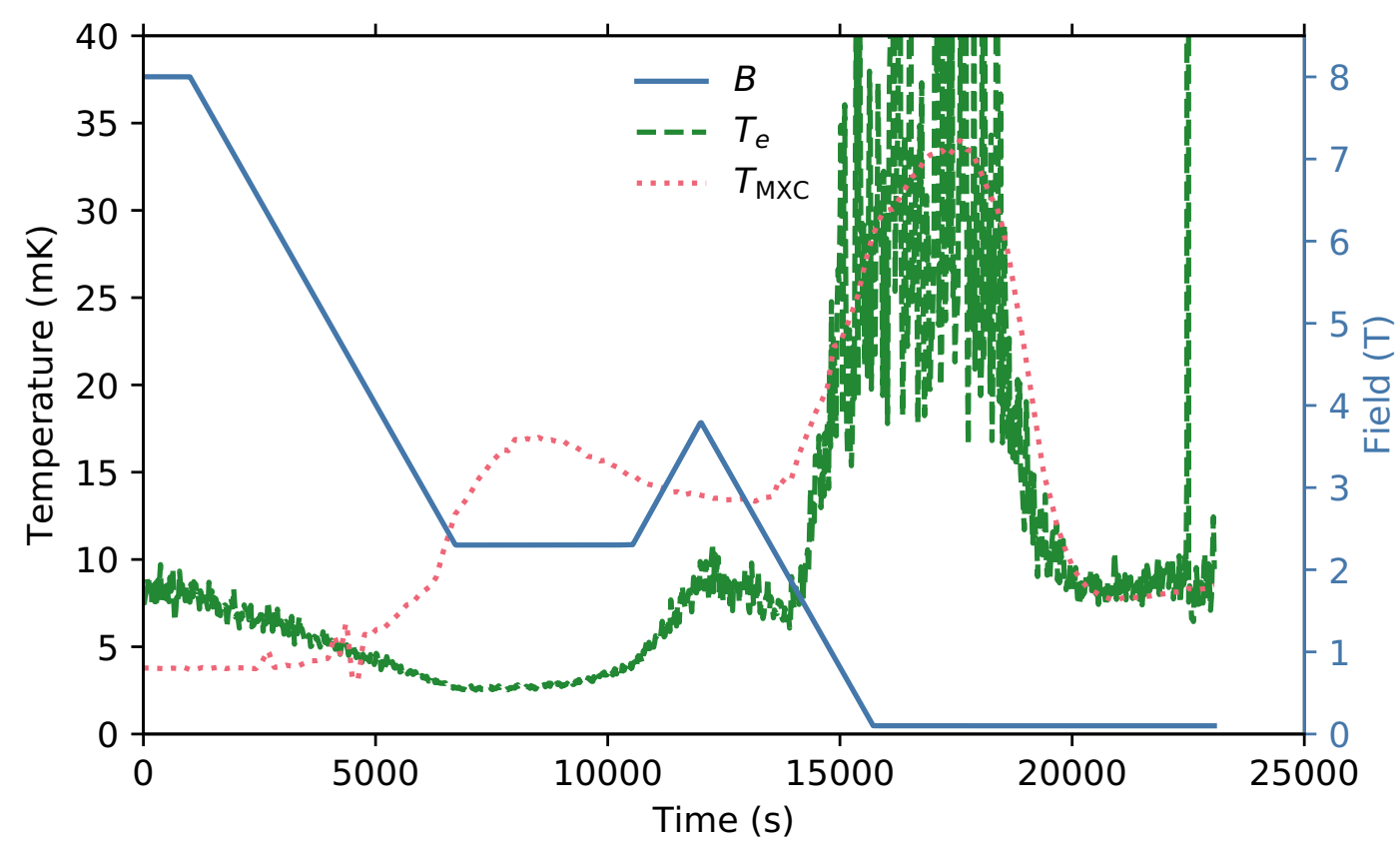

Figure 5.11: Fridge 4 Magnetic Heating Hysteresis. A peculiarity of demagnetisations on Fridge 4 is the heat released when demagnetising to new low fields. Note that when the magnetic field (solid blue line, right axis) drops below its previous value around $14000 \mathrm{~s}$ a significant amount of heating of the CBT (green dashed line) and MXC (red dotted line) occurs.

due a release of heat when demagnetising below $\approx 500 \mathrm{mT}$. Like in the current study, where we only conclude that this heat release occurs somewhere below $1.8 \mathrm{~T}$, the Aerogel study noted that the exact onset of this effect is difficult to judge. The Aerogel study concluded that the most likely origin of this heating was from some magnetic material inside the cell. However in this study, which has no such cell, an effect observed in the Aerogel study was reproduced: The heating shows hysteresis whereby the bulk of the heat is released when demagnetising below magnetic fields that have not yet been visited during the demagnetisation. This is shown in Figure 5.11.

Here the magnetic field, using a rate of $1 \mathrm{mT} \mathrm{s}^{-1}$, was first decreased from $8.0 \mathrm{~T}$ to $2.3 \mathrm{~T}$. During this, the MXC warmed from eddy current heating and the CBT cooled due to the demagnetisation of the on-chip copper, as expected. Secondly, the field was held constant at $2.3 \mathrm{~T}$. This allowed the MXC to begin to cool and the CBT to slowly begin to thermalise back to the MXC, again as expected. Thirdly, the field was increased to $3.8 \mathrm{~T}$ at the same rate. This also produced the expected outcome, with the CBT warming from the heat of magnetisation. The field was finally decreased to $0.1 \mathrm{~T}$. This initially produced the usual cooling of the CBT from demagnetisation, and the beginning ${ }^{1}$ of the warming of the MXC. After the

\footnotetext{
${ }^{1}$ Note that up to this point, the response of the MXC is always delayed, possibly due to the
} 
field drops below 2.3 T (where the ramp was paused earlier), the MXC and CBT immediately respond by quickly warming. This same hysteretic response is seen in Figure 6.15 in [137].

Given that this behaviour has now been seen with a completely different experiment mounted on Fridge 4, it is clear that this must be arising from the dilution refrigerator itself. In [137], this idea is considered, with one theory being that it is the result of the specific $\mathrm{CuNi}$ alloy the dilution refrigerator's heat exchangers are made from. In the CBT investigation, the heat exchangers were checked with a permanent magnet, and were found to be weakly magnetic, while those on the other dilution refrigerators in the lab are not. This gives rise to possibilities such as a magnetic phase transition occurring and hence releasing heat upon demagnetisation, or for some motion occurring as the field is swept leading to frictional heat production. The exact cause and reason for the observed hysteresis is, however, unknown and requires further work to diagnose.

In this study, several attempts were made to work around the issue. First, the $20 \mathrm{mK}$ shielding can was wrapped with $\mathrm{Mu}$-metal $^{2}$ in an attempt to reduce the flux from the solenoid through the heat exchangers. According to a giant magnetoresistance sensor installed near the heat exchangers, however, this made no difference to the field in this region, possibly because of the reduction in permeability $\mathrm{Mu}-$ metal experiences at low temperatures, or because of the field was sufficiently high to saturate it. Superconducting lead foil was also attempted for this shielding, which again made no difference, possibly because of the critical field being exceeded. Finally, an aluminium heat switch was added between the CBT package and the silver sinter cooling it. This heat switch was located in the compensated region of the main solenoid, and a small control solenoid was wound around it, allowing independent control of the thermal link to the package. This initially seemed promising, with the CBT able to be thermally isolated from the heated MXC while testing with the MXC resistive heater. However, during actual demagnetisations, this did not work since the eddy current heating of the silver package itself was unable to be dissipated, leading to another excessive heat leak into the CBT electrons. The conclusion of this was that it was unlikely anything could be done about the heat leak, making reaching any lower electron temperatures on the CBT impossible.

fact it is made of plastic meaning it takes time for heat from eddy current heating to reach it from elsewhere in the cryostat.

${ }^{2} \mathrm{Mu}$-metal is a ferromagnetic alloy, with exceptionally high permeability, used for magnetic shielding [138] 


\subsection{CBT Copper}

The discussion on heat leaks in Section 5.4 assumes the normal nuclear spin heat capacity for copper, given by Equation 5.5, as calculated with the accepted value of copper's internal magnetic dipole field $b=0.36 \mathrm{mT}$ [23]. However, for certain forms of copper with a large amount of internal strain, this value can be much bigger. It has been found previously [28] that one form of copper powder, made by hammering bulk copper into small flakes, has $b=350 \mathrm{mT}$. This made demagnetising below $\approx 1 \mathrm{mK}$ impossible and created an unexpectedly high heat capacity. A highly granular or amorphous form of copper would also explain the small effective volume for the electron-phonon coupling ( $\approx 10 \%$ of the true volume) found in the thermal modelling, since the mean free path for phonon-electron collisions will be constrained by the grain sizes $[108,109]$. It is also possible that some impurities in the copper may lead to additional heat leaks, for example through the ortho-para conversion of hydrogen [139].

The quality of the electroplated copper on the CBT is unknown in this regard, so in order to check for these effects, which may be significant factors in limiting the lowest temperatures obtained in this study, the copper refrigerant on the CBT was compared, using X-Ray powder diffraction (XRD) [140], with a sample of the high- $b$ copper powder and with a sample of the high-purity, annealed bulk copper used in Lancaster-style demagnetisation cells [30]. Powder diffraction was selected as it was anticipated that there would be many small grains $(\sim 100 \mathrm{~nm}$ long [141]) in random crystallographic orientations in the electroplated copper. The XRD diffraction pattern is shown in Figure 5.12.

From Figure 5.12, we see that all three samples have all the peaks expected for copper, although the intensities in the bulk copper are somewhat different to what would be anticipated, likely because of a preferred crystallographic direction being created during the annealing process, meaning that powder diffraction is not ideally suited to this sample. The powder sample is readily distinguishable from the bulk by the broader peaks and the additional, lower intensity, satellite peaks. The broader peaks in the powder were observed before in [28] and suggest the presence of some randomly distributed strain, introduced by the flake hammering process. This hammering process will also tend to distort the copper lattice from the cubic to the tetragonal system. This breaking of the cubic symmetry, from a face-centred-cubic (FCC) to a body-centred-tetragonal (BCT) lattice, leads to additional X-ray reflections being permitted, explaining the production of the satellite peaks. The angular shift of these peaks is consistent with a $-0.91 \%$ strain in the lattice. 


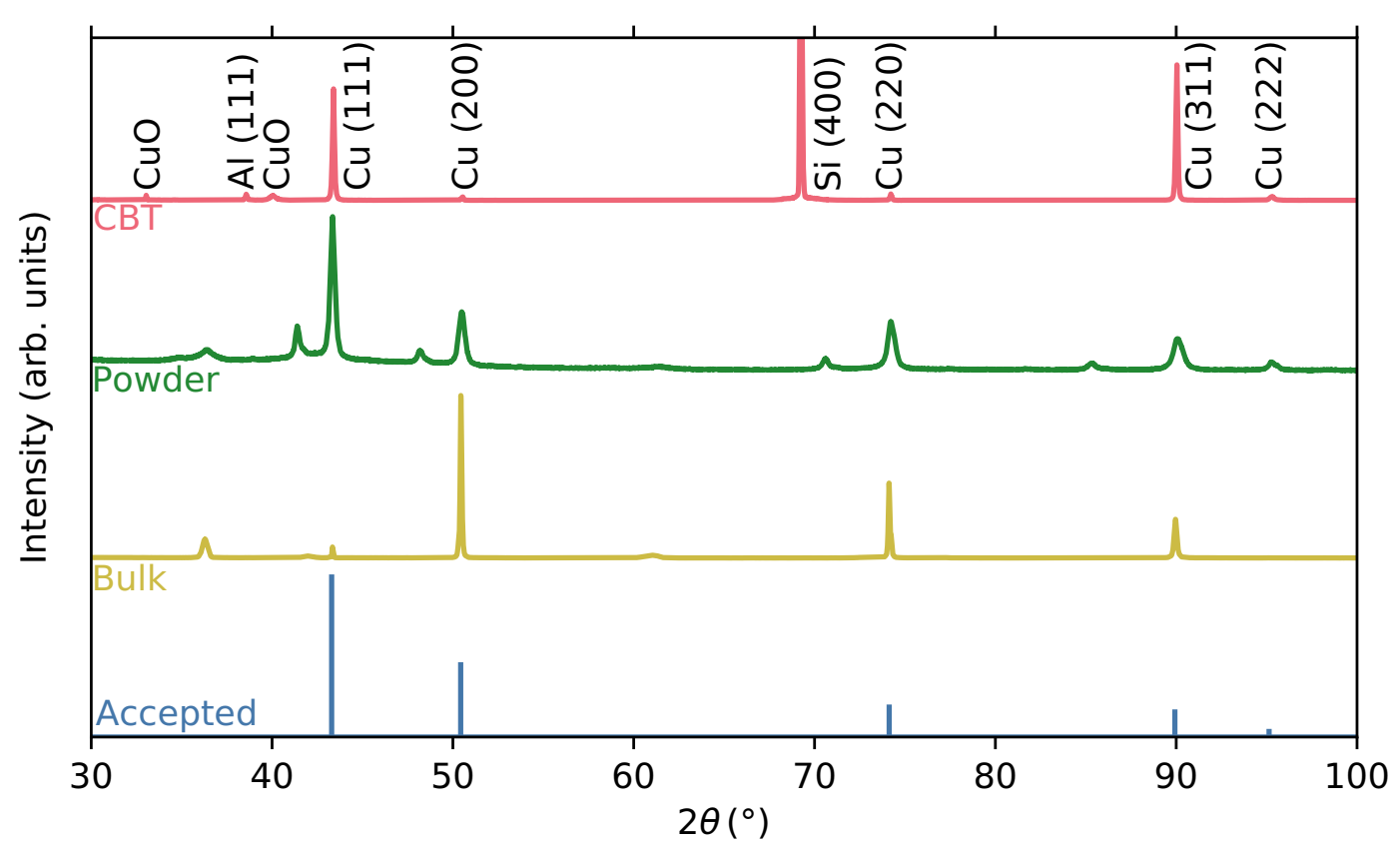

Figure 5.12: X-Ray Diffraction of the CBT Copper. Each line shows the normalised diffraction pattern of the labelled copper sample, as measured using $\mathrm{Cu} \mathrm{K} \mathrm{K}_{\alpha}$ radiation with wavelength $1.54 \AA$. To improve visibility, each line is offset from the others and the $\mathrm{Si}$ (400) peak has been clipped by a factor of $\approx 50$. The accepted XRD data for $\mathrm{Cu}$ is from [142]. This figure is redrawn from [110].

The signal from the CBT sample also contains additional peaks, though these are readily accountable for: The high intensity $\mathrm{Si}$ (400) peak is from the much thicker $(675 \mu \mathrm{m})$ silicon substrate. This is highly crystalline, meaning that a powder diffraction scan only shows a single peak. The Al (111) peak is the most intense aluminium peak [142], and is the only one visible here due to the aluminium layer under the copper being very thin $(250 \mathrm{~nm})$.

However, the narrow peaks and lack of satellite peaks in the CBT XRD pattern make it clear that overall, the CBT copper is more similar to the bulk copper than the powder, ruling out an increased value of $b$ through strain. Other than a small amount of $\mathrm{CuO}$ present, no impurities can be seen, however XRD is not sensitive to the effect of impurities such as $\mathrm{H}_{2}$. Further study using, for example X-ray photoelectron spectroscopy (XPS) [143] or secondary ion mass spectrometry (SIMS) [144] may be beneficial for revealing other impurities.

\subsection{Conclusions}

In conclusion, the successful CBT design, used in Chapter 3 for the first proof of concept testing into the application of nuclear demagnetisation refrigeration 
to on-chip electron cooling, has been modified by adding a significant amount of stray capacitance to each island. In theory, this has reduced the minimum electron temperature at which the CBT can perform thermometry down to $\approx$ $310 \mu \mathrm{K}$, although temperatures this low have not been measured in this study. Nevertheless, by reapplying the techniques developed for the study in Chapter 4 , the CBT electrons have been cooled to $(1.20 \pm 0.03) \mathrm{mK}$ which, to our knowledge, is the lowest device electron temperature achieved using only on-chip refrigerant, or any form of copper refrigerant. Improving on this, using only on-chip magnetic cooling, will require moving to a system which results in smaller heat leaks. 
BLANK 


\section{Chapter 6}

\section{Summary and Outlook}

This thesis has described some of the very latest work in on-chip cooling of the electrons within a nanoelectronic device. This particular study has been focussed on the use of adiabatic nuclear demagnetisation refrigeration [23], using only on-chip copper refrigerant, which was electroplated in direct electrical contact with the conductive elements on the nanoelectronic device. This approach permitted the construction of a simple cooling platform, with no requirements for large amounts of external copper and superconducting heat switches. Instead, the sample to be cooled, with copper refrigerant applied, was demagnetised inside a dilution refrigerator where the weak electron-phonon coupling provides the thermal isolation necessary for the device electrons to be cooled below the temperature of their host lattice, and the temperature of the dilution refrigerator.

Before this work, the majority of on-chip cooler studies were electronic in nature, relying on the creation of energy filters using junctions or quantum dots [69]. The main motivation of these studies was the replacement of the dilution refrigerator, not the creation of new techniques for reaching new low temperatures to enable the study of new physical phenomena or to improve existing quantum devices. Initial work on reaching new low electron temperatures commenced before the work described in this thesis, with an experiment on using a CBT designed to thermalise well to its cooling platform. This was cooled first in a cryogen free dilution refrigerator to $7.2 \mathrm{mK}$, then in a wet dilution refrigerator to $4.0 \mathrm{mK}$ and finally to $3.7 \mathrm{mK}$ when immersed in the liquid ${ }^{3} \mathrm{He}-{ }^{4} \mathrm{He}$ mixture $[49,127]$. At the time this was a record low temperature for electrons in a nanoelectronic device.

Following this, independently of the work presented here, magnetic cooling within a nanoelectronic device was first reported in manganese doped aluminium. This doping was used for the suppression of superconductivity in aluminium, but was 
discovered to be usable for demagnetisation cooling of the device electrons to $140 \mathrm{mK}$ [83], giving another potential candidate for the replacement of the dilution refrigerator.

In parallel with this first report of on-chip magnetic cooling, the first proof of concept work of on-chip nuclear demagnetisation cooling using copper refrigerant, as described in Chapter 3 of this thesis, took place. In this work, a variation of the CBT design used for the immersion cooling study was used with electroplated copper refrigerant on the CBT islands. This allowed the device electrons to be cooled down to $4.5 \mathrm{mK}$, with the device mounted in a commercial dry dilution refrigerator with a base temperature of $7 \mathrm{mK}$. While the base electron temperature achieved here was higher than that achieved in the immersion cooling study, it was well below the base temperature of the majority of commercial dilution refrigerators and demonstrated the effectiveness of the technique. This work was published in $[88]$.

This work was followed by an attempt at replicating the technique using one of the custom-built Lancaster dilution refrigerators to provide the pre-cooling, as described in Chapter 4 of this thesis. This work required the development of a number of new techniques in order to enable the measurement of a CBT at new low temperatures. While these techniques were effective, the design of this CBT was unsuited to the low temperatures achieved in this part of the study due to it having too high a charging energy. This lead to inaccurate thermometry and a large uncertainty in the final electron temperature, $(1.1 \pm 0.4) \mathrm{mK}$.

Subsequently, as described in Chapter 5 of this thesis, the CBT design was modified in order to increase the stray capacitance of each island, hence reducing the charging energy and producing a CBT capable of primary thermometry down to $\approx 300 \mu \mathrm{K}$. To the best of our knowledge, this is the lowest temperature capable CBT produced to date. Demagnetisation refrigeration was then performed on this device in a different Lancaster dilution refrigerator, utilizing the techniques developed in Chapter 4. This resulted in a minimum electron temperature of $(1.20 \pm 0.03) \mathrm{mK}$, to our knowledge the lowest on-chip electron temperature reached using copper refrigerant, and also the lowest on-chip electron temperature reached using only on-chip refrigerant of any material. This temperature was found to be limited by the heat leaks into the system, predominantly caused by a peculiarity of the particular dilution refrigerator used. This work, in combination with the techniques developed in Chapter 4 are to be published in [110].

In other laboratories, interest in producing new low electron temperatures in nanoelectronic devices has expanded. In Basel, a new on-and-off chip copper demag- 
netisation cooling platform was designed [145] and tested with a CBT, giving minimum electron temperatures initially of $2.8 \mathrm{mK}[134,146]$ and then of $1.8 \mathrm{mK}$ [147]. Following this, on-chip cooling using indium refrigerant, useful for its high heat capacity and strong coupling between the nuclear spins and electrons, has been explored. First with on-chip only indium refrigerant, yielding a minimum electron temperature of $3.2 \mathrm{mK}$ [103], and then with on-and-off chip indium refrigerant, allowing a temperature of $(420 \pm 40) \mu \mathrm{K}[50]$ to be reached, opening the door to sub-millikelvin nanoelectronics.

The next stages for this technology are its expansion to systems other than the CBT. The CBT was well suited to the proof of concept investigations detailed here since it is a primary thermometer of its own electron temperature, which is what we desire to cool. For the future, employing this technique on other devices to allow the exploration of new physics, is more appealing. These devices could be cooled using copper or indium refrigerant, or to enable the use of non-metallic structures, demagnetisation of doped semiconductors [148] could be attempted. One example of a quantum transport measurement was made in [50] by measuring the CBT conductance vs. bias voltage curve while cold, proving that similar measurements will be possible with the heat capacity available in their system. However, measurements in a new system that are enabled by the new low temperatures developed here should be the eventual goal.

An investigation that could be straightforwardly integrated with the on-chip cooling technique is one into the appearance of a Kondo-like temperature dependence on the resistivity of certain metal alloys which do not contain magnetic impurities. These alloys feature potential wells at random locations throughout the material as a result of a disordered distribution of the alloying element [149]. The interaction of the conduction electrons with these potential wells results in the classic logarithmic temperature dependence of resistivity, as seen in the Kondo effect [150].

This phenomenon has recently been observed to occur in $\mathrm{Au}-\mathrm{Ge}$ alloys, containing $\approx 10 \% \mathrm{Au}$, which have been fabricated into a simple Hall bar geometry [151]. In this particular case, the exact form of the resistance vs. temperature curve is such that sensors fabricated from this material are candidates for an easy to measure resistance thermometer capable of operating from room temperature down to at least $200 \mathrm{mK}$. Further cooling these alloys down to $\sim 1 \mathrm{mK}$ in order to investigate their effectiveness for thermometry at lower temperatures, or indeed explore any different behaviour they may have at these temperatures, would be worthwhile.

The $1 \mathrm{~mm}$ long by $80 \mu \mathrm{m}$ wide Hall bars fabricated in [151] from $200 \mathrm{~nm}$ thick films 
are of comparable dimensions to the CBT islands on the devices investigated in this thesis. Therefore, the on-chip cooling technique could be applied to these $\mathrm{Au}-\mathrm{Ge}$ alloys by electroplating copper or indium refrigerant onto several different samples which could then be measured using four resistive connections, giving the required thermal isolation from the warm surroundings. Since this Kondo-like temperature dependence provides only secondary thermometry, this structure could be integrated onto a chip also holding a CBT which could be used for calibration.

Should cooling these samples prove successful, and their thermometry be accurate at low temperatures, these simple resistive thermometers could then be integrated into other on-chip cooling platforms to allow thermometry when cooling other samples which lack their own capability for temperature readout. Such an integrated thermometer would be better suited to this task than a CBT since the measurement is simpler to perform and the device is easier to fabricate. Going forward, this integration could be performed with, for example, a charge qubit chip, allowing an investigation into the dependence of the coherence time on the electron temperature. 


\section{Bibliography}

[1] Aivon Oy. pyCBT, June 2014. URL

https://github.com/AivonOy/pyCBT.

[2] K. S. Novoselov, Z. Jiang, Y. Zhang, S. V. Morozov, H. L. Stormer, U. Zeitler, J. C. Maan, G. S. Boebinger, P. Kim, and A. K. Geim. Room-temperature quantum Hall effect in graphene. Science, 315(5817): 1379-1379, March 2007. doi:10.1126/science.1137201.

[3] M. K. Wu, J. R. Ashburn, C. J. Torng, P. H. Hor, R. L. Meng, L. Gao, Z. J. Huang, Y. Q. Wang, and C. W. Chu. Superconductivity at 93 K in a new mixed-phase $\mathrm{Y}-\mathrm{Ba}-\mathrm{Cu}-\mathrm{O}$ compound system at ambient pressure. Physical Review Letters, 58(9):908-910, March 1987. doi:10.1103/PhysRevLett.58.908.

[4] J. Clarke and A. I. Braginski. The SQUID handbook. Wiley-VCH, 2004. ISBN 3-527-40229-2. doi:10.1002/3527603646.

[5] O. V. Lounasmaa. Dilution refrigeration. Journal of Physics E: Scientific Instruments, 12(8):668-675, August 1979. doi:10.1088/0022-3735/12/8/001.

[6] D. J. Cousins, S. N. Fisher, A. M. Guénault, R. P. Haley, I. E. Miller, G. R. Pickett, G. N. Plenderleith, P. Skyba, P. Y. A. Thibault, and M. G. Ward. An advanced dilution refrigerator designed for the new Lancaster microkelvin facility. Journal of Low Temperature Physics, 114(5):547-570, March 1999. doi:10.1023/A:1021862406629.

[7] K. Uhlig. Cryogen-free dilution refrigerators. Journal of Physics: Conference Series, 400(5):052039, December 2012. doi:10.1088/1742-6596/400/5/052039.

[8] J. Clarke and F. K. Wilhelm. Superconducting quantum bits. Nature, 453 (7198):1031-1042, June 2008. doi:10.1038/nature07128.

[9] G. Wendin. Quantum information processing with superconducting 
circuits: a review. Reports on Progress in Physics, 80(10):106001, September 2017. doi:10.1088/1361-6633/aa7e1a.

[10] P. Krantz, M. Kjaergaard, F. Yan, T. P. Orlando, S. Gustavsson, and W. D. Oliver. A quantum engineer's guide to superconducting qubits. Applied Physics Reviews, 6(2):021318, June 2019. doi:10.1063/1.5089550.

[11] S. Nakamura, Yu. A. Pashkin, J. S. Tsai, N.-H. Kaneko, et al. SINIS turnstile for quantum current standards. In Precision Electromagnetic Measurements (CPEM), pages 660-661. IEEE, October 2014. doi:10.1109/CPEM.2014.6898558.

[12] R. N. Jabdaraghi, J. T. Peltonen, D. S. Golubev, and J. P. Pekola. Magnetometry with low-resistance proximity Josephson junction. Journal of Low Temperature Physics, 191(5):344-353, June 2018. doi:10.1007/s10909-018-1863-x.

[13] M. L. Roukes, M. R. Freeman, R. S. Germain, R. C. Richardson, and M. B. Ketchen. Hot electrons and energy transport in metals at millikelvin temperatures. Physical Review Letters, 55(4):422-425, July 1985. doi:10.1103/PhysRevLett.55.422.

[14] L. A. Tracy, D. R. Luhman, S. M. Carr, N. C. Bishop, G. A. Ten Eyck, T. Pluym, J. R. Wendt, M. P. Lilly, and M. S. Carroll. Single shot spin readout using a cryogenic high-electron-mobility transistor amplifier at sub-Kelvin temperatures. Applied Physics Letters, 108(6):063101, February 2016. doi:10.1063/1.4941421.

[15] T. Albash, V. Martin-Mayor, and I. Hen. Temperature scaling law for quantum annealing optimizers. Physical Review Letters, 119(11):110502, September 2017. doi:10.1103/PhysRevLett.119.110502.

[16] Yu. A. Pashkin, O. Astafiev, T. Yamamoto, Y. Nakamura, and J. S. Tsai. Josephson charge qubits: a brief review. Quantum Information Processing, 8(2):55-80, February 2009. doi:10.1007/s11128-009-0101-5.

[17] A. Dey and S. Yarlagadda. Temperature dependence of long coherence times of oxide charge qubits. Scientific Reports, 8(1):3487, February 2018. doi:10.1038/s41598-018-21767-2.

[18] J. P. Pekola, O.-P. Saira, V. F. Maisi, A. Kemppinen, M. Möttönen, Yu. A. Pashkin, and D. V. Averin. Single-electron current sources: toward a 
refined definition of the ampere. Reviews of Modern Physics, 85(4): 1421-1472, October 2013. doi:10.1103/RevModPhys.85.1421.

[19] S. Nakamura, Yu. A. Pashkin, J. S. Tsai, and N.-H. Kaneko. Temperature dependence of single-electron pumping using a SINIS turnstile. Physica $C$ : Superconductivity, 504:93-96, September 2014. doi:10.1016/j.physc.2014.02.023.

[20] F. Giazotto, T. T. Heikkilä, A. Luukanen, A. M. Savin, and J. P. Pekola. Opportunities for mesoscopics in thermometry and refrigeration: physics and applications. Reviews of Modern Physics, 78(1):217-274, March 2006. doi:10.1103/RevModPhys.78.217.

[21] P. Das, R. Bruyn de Ouboter, and K. W. Taconis. A realization of a London-Clarke-Mendoza type refrigerator. In J. G. Daunt, D. O. Edwards, F. J. Milford, and M. Yaqub, editors, Low Temperature Physics LT9, pages 1253-1255, Boston, MA, September 1965. Springer US. doi:10.1007/978-1-4899-6443-4_133.

[22] K. Uhlig and W. Hehn. 3He/4He dilution refrigerator with Gifford-McMahon precooling. Cryogenics, 33(11):1028-1031, November 1993. doi:10.1016/0011-2275(93)90204-2.

[23] F. Pobell. Matter and methods at low temperatures. Springer-Verlag, Berlin, third edition, 2007. ISBN 978-3-540-46360-3. doi:10.1007/978-3-540-46360-3.

[24] E. Warburg and L. Hönig. Ueber die Wärme, welche durch periodisch wechselnde magnetisirende Kräfte im Eisen erzeugt wird. Annalen der Physik, 256(12):814-835, August 1883. doi:10.1002/andp.18832561217.

[25] W. J. Huiskamp and O. V. Lounasmaa. Ultralow temperatures - how and why. Reports on Progress in Physics, 36(4):423-496, April 1973. doi:10.1088/0034-4885/36/4/002.

[26] J. P. Eisenstein, G. W. Swift, and R. E. Packard. A microprocessor controller for a nuclear demagnetization refrigerator. Cryogenics, 19(11): 666-668, November 1979. doi:10.1016/0011-2275(79)90069-9.

[27] J. Bardeen, G. Rickayzen, and L. Tewordt. Theory of the thermal conductivity of superconductors. Physical Review, 113(4):982-994, February 1959. doi:10.1103/PhysRev.113.982.

[28] D. I. Bradley, A. M. Guénault, V. Keith, C. J. Kennedy, I. E. Miller, S. G. 
Mussett, G. R. Pickett, and W. P. Pratt. New methods for nuclear cooling into the microkelvin regime. Journal of Low Temperature Physics, 57(3): 359-390, November 1984. doi:10.1007/BF00681199.

[29] J. Korringa. Nuclear magnetic relaxation and resonnance line shift in metals. Physica, 16(7):601-610, August 1950. doi:10.1016/0031-8914(50)90105-4.

[30] G. R. Pickett. Microkelvin physics. Reports on Progress in Physics, 51(10): 1295-1340, October 1988. doi:10.1088/0034-4885/51/10/001.

[31] N. Kurti, F. N. H. Robinson, F. Simon, and D. A. Spohr. Nuclear cooling. Nature, 178(4531):450-453, September 1956. doi:10.1038/178450a0.

[32] M. Kubota, H. R. Folle, Ch. Buchal, R. M. Mueller, and F. Pobell. Nuclear susceptibility and thermodynamic analysis of the nuclear specific heat of PrNi $_{5}$. Physica B+C, 108(1):1093-1094, September 1981. doi:10.1016/0378-4363(81)90850-0.

[33] G. Batey, A. Casey, M. N. Cuthbert, A. J. Matthews, J. Saunders, and A. Shibahara. A microkelvin cryogen-free experimental platform with integrated noise thermometry. New Journal of Physics, 15:113034, November 2013. doi:10.1088/1367-2630/15/11/113034.

[34] M. Kubota, H. R. Folle, Ch. Buchal, R. M. Mueller, and F. Pobell. Nuclear magnetic ordering in $\mathrm{PrNi}_{5}$ at $0.4 \mathrm{mK}$. Physical Review Letters, 45(22): 1812-1815, December 1980. doi:10.1103/PhysRevLett.45.1812.

[35] G. J. Ehnholm, J. P. Ekström, J. F. Jacquinot, M. T. Loponen, O. V. Lounasmaa, and J. K. Soini. Evidence for nuclear antiferromagnetism in copper. Physical Review Letters, 42(25):1702-1705, June 1979. doi:10.1103/PhysRevLett.42.1702.

[36] H. R. Folle, M. Kubota, Ch. Buchal, R. M. Mueller, and F. Pobell. Nuclear refrigeration properties of PrNi5. Zeitschrift für Physik B Condensed Matter, 41(3):223-228, September 1981. doi:10.1007/BF01294426.

[37] C. Enss and S. Hunklinger. Low-temperature physics. Springer Science \& Business Media, Heidelberg, 2005. ISBN 978-3-540-23164-6. doi:10.1007/b137878.

[38] J. P. Pekola, K. P. Hirvi, J. P. Kauppinen, and M. A. Paalanen. Thermometry by arrays of tunnel junctions. Physical Review Letters, 73 (21):2903-2906, November 1994. doi:10.1103/PhysRevLett.73.2903. 
[39] U. Meirav and E. B. Foxman. Single-electron phenomena in semiconductors. Semiconductor Science and Technology, 11(3):255-284, March 1996. doi:10.1088/0268-1242/11/3/003.

[40] Sh. Farhangfar, K. P. Hirvi, J. P. Kauppinen, J. P. Pekola, J. J. Toppari, D. V. Averin, and A. N. Korotkov. One dimensional arrays and solitary tunnel junctions in the weak Coulomb blockade regime: CBT thermometry. Journal of Low Temperature Physics, 108(1):191-215, July 1997. doi:10.1007/BF02396821.

[41] M. A. Kastner. The single-electron transistor. Reviews of Modern Physics, 64(3):849-858, July 1992. doi:10.1103/RevModPhys.64.849.

[42] H. Grabert and M. H. Devoret. Single charge tunneling: Coulomb blockade phenomena in nanostructures, volume 294. Springer Science \& Business Media, 1992. ISBN 978-1-4757-2168-3. doi:10.1007/978-1-4757-2166-9.

[43] J. P. Pekola, L. J. Taskinen, and Sh. Farhangfar. One- and two-dimensional tunnel junction arrays in weak Coulomb blockade regime: absolute accuracy in thermometry. Applied Physics Letters, 76(25): 3747-3749, June 2000. doi:10.1063/1.126770.

[44] D. V. Averin and K. K. Likharev. Single electronics: a correlated transfer of single electrons and Cooper pairs in systems of small tunnel junctions. Mesoscopic phenomena in solids, 30:173-271, July 1991. doi:10.1007/978-1-4899-3689-9_24.

[45] C. B. Duke. Tunneling in solids. Solid state physics. Supplement 10. Academic Press, New York, 1969. ISBN 978-0-12-607770-4.

[46] C. Wasshuber. Computational single-electronics. Springer Science \& Business Media, 2001. ISBN 978-3-7091-6257-6. doi:10.1007/978-3-7091-6257-6.

[47] K. P. Hirvi, J. P. Kauppinen, A. N. Korotkov, M. A. Paalanen, and J. P. Pekola. Arrays of normal metal tunnel junctions in weak Coulomb blockade regime. Applied Physics Letters, 67(14):2096-2098, October 1995. doi:10.1063/1.115090.

[48] A. V. Feshchenko, M. Meschke, D. Gunnarsson, M. Prunnila, L. Roschier, J. S. Penttilä, and J. P. Pekola. Primary thermometry in the intermediate Coulomb blockade regime. Journal of Low Temperature Physics, 173(1): 36-44, April 2013. doi:10.1007/s10909-013-0874-x. 
[49] D. I. Bradley, R. E. George, D. Gunnarsson, R. P. Haley, H. Heikkinen, Yu. A. Pashkin, J. Penttila, J. R. Prance, M. Prunnila, L. Roschier, and M. Sarsby. Nanoelectronic primary thermometry below $4 \mathrm{mK}$. Nature Communications, 7:10455, January 2016. doi:10.1038/ncomms10455.

[50] M. Sarsby, N. Yurttagül, and A. Geresdi. 500 microkelvin nanoelectronics. arXiv e-prints, arXiv:1903.01388 [cond-mat.mes-hall], March 2019. URL https://arxiv.org/abs/1903.01388.

[51] M. Meschke, A. Kemppinen, and J. P. Pekola. Accurate Coulomb blockade thermometry up to 60 kelvin. Philosophical Transactions of the Royal Society A - Mathematical Physical and Engineering Sciences, 374(2064): 20150052, March 2016. doi:10.1098/rsta.2015.0052.

[52] O. Hahtela, E. Mykkänen, A. Kemppinen, M. Meschke, M. Prunnila, D. Gunnarsson, L. Roschier, J. Penttilä, and J. Pekola. Traceable Coulomb blockade thermometry. Metrologia, 54(1):69-76, December 2016. doi:10.1088/1681-7575/aa4f84.

[53] F. C. Wellstood, C. Urbina, and J. Clarke. Hot-electron effects in metals. Physical Review B, 49(9):5942-5955, March 1994. doi:10.1103/PhysRevB.49.5942.

[54] P. M. Echternach, M. R. Thoman, C. M. Gould, and H. M. Bozler. Electron-phonon scattering rates in disordered metallic films below $1 \mathrm{~K}$. Physical Review B, 46(16):10339-10344, October 1992. doi:10.1103/PhysRevB.46.10339.

[55] L. Casparis, M. Meschke, D. Maradan, A. C. Clark, C. P. Scheller, K. K. Schwarzwälder, J. P. Pekola, and D. M. Zumbühl. Metallic Coulomb blockade thermometry down to $10 \mathrm{mK}$ and below. Review of Scientific Instruments, 83(8):083903, August 2012. doi:10.1063/1.4744944.

[56] G. L. Pollack. Kapitza resistance. Reviews of Modern Physics, 41(1):48-81, January 1969. doi:10.1103/RevModPhys.41.48.

[57] R. J. Robertson, F. Guillon, and J. P. Harrison. Properties of sintered submicron copper and silver powders and their relation to low temperature heat exchangers. Canadian Journal of Physics, 61(2):164-176, February 1983. doi:10.1139/p83-023.

[58] Y. C. Chung, M. Heiblum, and V. Umansky. Scattering of bunched 
fractionally charged quasiparticles. Physical Review Letters, 91(21):216804, November 2003. doi:10.1103/PhysRevLett.91.216804.

[59] R. M. Potok, I. G. Rau, Hadas Shtrikman, Yuval Oreg, and D. Goldhaber-Gordon. Observation of the two-channel Kondo effect. Nature, 446(7132):167-171, March 2007. doi:10.1038/nature05556.

[60] Z. Iftikhar, A. Anthore, S. Jezouin, F. D. Parmentier, Y. Jin, A. Cavanna, A. Ouerghi, U. Gennser, and F. Pierre. Primary thermometry triad at $6 \mathrm{mK}$ in mesoscopic circuits. Nature Communications, 7:12908, September 2016. doi:10.1038/ncomms12908.

[61] C. P. Scheller, S. Heizmann, K. Bedner, D. Giss, M. Meschke, D. M. Zumbühl, J. D. Zimmerman, and A. C. Gossard. Silver-epoxy microwave filters and thermalizers for millikelvin experiments. Applied Physics Letters, 104(21):211106, May 2014. doi:10.1063/1.4880099.

[62] J. M. Hergenrother, J. G. Lu, M. T. Tuominen, D. C. Ralph, and M. Tinkham. Photon-activated switch behavior in the single-electron transistor with a superconducting island. Physical Review B, 51(14): 9407-9410, April 1995. doi:10.1103/PhysRevB.51.9407.

[63] J. P. Pekola, V. F. Maisi, S. Kafanov, N. Chekurov, A. Kemppinen, Yu. A. Pashkin, O.-P. Saira, M. Möttönen, and J. S. Tsai. Environment-assisted tunneling as an origin of the Dynes density of states. Physical Review Letters, 105(2):026803, July 2010. doi:10.1103/PhysRevLett.105.026803.

[64] I. Todoshchenko, J.-P. Kaikkonen, R. Blaauwgeers, P. J. Hakonen, and A. Savin. Dry demagnetization cryostat for sub-millikelvin helium experiments: refrigeration and thermometry. Review of Scientific Instruments, 85(8):085106, August 2014. doi:10.1063/1.4891619.

[65] F. Mueller, R. N. Schouten, M. Brauns, T. Gang, W. H. Lim, N. S. Lai, A. S. Dzurak, W. G. van der Wiel, and F. A. Zwanenburg. Printed circuit board metal powder filters for low electron temperatures. Review of Scientific Instruments, 84(4):044706, April 2013. doi:10.1063/1.4802875.

[66] W. Pan, J.-S. Xia, V. Shvarts, D. E. Adams, H. L. Stormer, D. C. Tsui, L. N. Pfeiffer, K. W. Baldwin, and K. W. West. Exact quantization of the even-denominator fractional quantum hall state at $\nu=5 / 2$ Landau level filling factor. Physical Review Letters, 83(17):3530-3533, October 1999. doi:10.1103/PhysRevLett.83.3530. 
[67] J. S. Xia, E. D. Adams, V. Shvarts, W. Pan, H. L. Stormer, and D. C. Tsui. Ultra-low-temperature cooling of two-dimensional electron gas. Physica B: Condensed Matter, 280(1):491-492, May 2000. doi:10.1016/S0921-4526(99)01843-8.

[68] N. Samkharadze, A. Kumar, M. J. Manfra, L. N. Pfeiffer, K. W. West, and G. A. Csáthy. Integrated electronic transport and thermometry at milliKelvin temperatures and in strong magnetic fields. Review of Scientific Instruments, 82(5):053902, May 2011. doi:10.1063/1.3586766.

[69] J. T. Muhonen, M. Meschke, and J. P. Pekola. Micrometre-scale refrigerators. Reports on Progress in Physics, 75(4):046501, March 2012. doi:10.1088/0034-4885/75/4/046501.

[70] M. Nahum, T. M. Eiles, and John M. Martinis. Electronic microrefrigerator based on a normal-insulator-superconductor tunnel junction. Applied Physics Letters, 65(24):3123-3125, December 1994. doi:10.1063/1.112456.

[71] M. M. Leivo, A. J. Manninen, and J. P. Pekola. Microrefrigeration by normal-metal/insulator/superconductor tunnel junctions. Applied Superconductivity, 5(7-12):227-233, December 1997. doi:10.1016/S0964-1807(97)00049-5.

[72] O. Quaranta, P. Spathis, F. Beltram, and F. Giazotto. Cooling electrons from 1 to $0.4 \mathrm{~K}$ with V-based nanorefrigerators. Applied Physics Letters, 98 (3):032501, January 2011. doi:10.1063/1.3544058.

[73] A. M. Savin, M. Prunnila, P. P. Kivinen, J. P. Pekola, J. Ahopelto, and A. J. Manninen. Efficient electronic cooling in heavily doped silicon by quasiparticle tunneling. Applied Physics Letters, 79(10):1471-1473, August 2001. doi:10.1063/1.1399313.

[74] M. J. Prest, J. T. Muhonen, M. Prunnila, D. Gunnarsson, V. A. Shah, J. S. Richardson-Bullock, A. Dobbie, M. Myronov, R. J. H. Morris, T. E. Whall, E. H. C. Parker, and D. R. Leadley. Strain enhanced electron cooling in a degenerately doped semiconductor. Applied Physics Letters, 99(25):251908, December 2011. doi:10.1063/1.3670330.

[75] H. L. Edwards, Q. Niu, and A. L. de Lozanne. A quantum-dot refrigerator. Applied Physics Letters, 63(13):1815-1817, September 1993. doi:10.1063/1.110672.

[76] H. L. Edwards, Q. Niu, G. A. Georgakis, and A. L. de Lozanne. Cryogenic 
cooling using tunneling structures with sharp energy features. Physical Review B, 52(8):5714-5736, August 1995. doi:10.1103/PhysRevB.52.5714.

[77] J. R. Prance, C. G. Smith, J. P. Griffiths, S. J. Chorley, D. Anderson, G. A. C. Jones, I. Farrer, and D. A. Ritchie. Electronic refrigeration of a two-dimensional electron gas. Physical Review Letters, 102(14):146602, April 2009. doi:10.1103/PhysRevLett.102.146602.

[78] J. P. Pekola, A. J. Manninen, M. M. Leivo, K. Arutyunov, J. K. Suoknuuti, T. I. Suppula, and B. Collaudin. Microrefrigeration by quasiparticle tunnelling in NIS and SIS junctions. Physica B: Condensed Matter, 280(1): 485-490, May 2000. doi:10.1016/S0921-4526(99)01842-6.

[79] P. J. Lowell, G. C. O’Neil, J. M. Underwood, and J. N. Ullom. Macroscale refrigeration by nanoscale electron transport. Applied Physics Letters, 102 (8):082601, February 2013. doi:10.1063/1.4793515.

[80] G. C. O’Neil, P. J. Lowell, J. M. Underwood, and J. N. Ullom. Measurement and modeling of a large-area normal-metal/insulator/superconductor refrigerator with improved cooling. Physical Review B, 85(13):134504, April 2012. doi:10.1103/PhysRevB.85.134504.

[81] H. Q. Nguyen, M. Meschke, H. Courtois, and J. P. Pekola. Sub-50-mK electronic cooling with large-area superconducting tunnel junctions. Physical Review Applied, 2(5):054001, November 2014. doi:10.1103/PhysRevApplied.2.054001.

[82] D. Gunnarsson, J. S. Richardson-Bullock, M. J. Prest, H. Q. Nguyen, A. V. Timofeev, V. A. Shah, T. E. Whall, E. H. C. Parker, D. R. Leadley, M. Myronov, and M. Prunnila. Interfacial engineering of semiconductor-superconductor junctions for high performance micro-coolers. Scientific Reports, 5:17398, December 2015. doi:10.1038/srep17398.

[83] C. Ciccarelli, R. P. Campion, B. L. Gallagher, and A. J. Ferguson. Intrinsic magnetic refrigeration of a single electron transistor. Applied Physics Letters, 108(5):053103, February 2016. doi:10.1063/1.4941289.

[84] G. J. Dolan. Offset masks for lift-off photoprocessing. Applied Physics Letters, 31(5):337-339, September 1977. doi:10.1063/1.89690.

[85] D. Shen, R. Zhu, W. Xu, J. Chang, Z. Ji, G. Sun, C. Cao, and J. Chen. 
Character and fabrication of $\mathrm{Al} / \mathrm{Al} 2 \mathrm{O} 3 / \mathrm{Al}$ tunnel junctions for qubit application. Chinese Science Bulletin, 57(4):409-412, February 2012. doi:10.1007/s11434-011-4821-4.

[86] S. T. Ruggiero, A. Williams, W. H. Rippard, A. Clark, S. W. Deiker, L. R. Vale, and J. N. Ullom. Dilute Al-Mn alloys for low-temperature device applications. Journal of Low Temperature Physics, 134(3):973-984, February 2004. doi:10.1023/B:JOLT.0000013212.61515.56.

[87] G. R. Pickett. Cooling metals to the microkelvin regime, then and now. Physica B: Condensed Matter, 280(1-4):467-473, May 2000. doi:10.1016/S0921-4526(99)01838-4.

[88] D. I. Bradley, A. M. Guénault, D. Gunnarsson, R. P. Haley, S. Holt, A. T. Jones, Yu. A. Pashkin, J. Penttilä, J. R. Prance, M. Prunnila, and L. Roschier. On-chip magnetic cooling of a nanoelectronic device. Scientific Reports, 7:45566, April 2017. doi:10.1038/srep45566.

[89] M. Prunnila, M. Meschke, D. Gunnarsson, S. Enouz-Vedrenne, J. M. Kivioja, and J. P. Pekola. Ex situ tunnel junction process technique characterized by Coulomb blockade thermometry. Journal of Vacuum Science \& Technology B, 28(5):1026-1029, September 2010. doi:10.1116/1.3490406.

[90] S. Caplan and G. Chanin. Critical-field study of superconducting aluminum. Physical Review, 138(5A):1428-1433, May 1965. doi:10.1103/PhysRev.138.A1428.

[91] P. M. Tedrow and R. Meservey. Critical magnetic field of very thin superconducting aluminum films. Physical Review B, 25(1):171-178, January 1982. doi:10.1103/PhysRevB.25.171.

[92] O. M. Hahtela, M. Meschke, A. Savin, D. Gunnarsson, M. Prunnila, J. S. Penttilä, L. Roschier, M. Heinonen, A. Manninen, and J. P. Pekola. Investigation of uncertainty components in Coulomb blockade thermometry. AIP Conference Proceedings, 1552(1):142-147, September 2013. doi:10.1063/1.4819529.

[93] J. P. Pekola, T. Holmqvist, and M. Meschke. Primary tunnel junction thermometry. Physical Review Letters, 101(20):206801, November 2008. doi:10.1103/PhysRevLett.101.206801.

[94] R. Koch. Stress in evaporated and sputtered thin films - a comparison. 
Surface and Coatings Technology, 204(12):1973-1982, March 2010. doi:10.1016/j.surfcoat.2009.09.047.

[95] L. Roschier, D. Gunnarsson, M. Meschke, A. Savin, J. S. Penttilä, and M. Prunnila. Effect of on-chip filter on Coulomb blockade thermometer. Journal of Physics: Conference Series, 400(5):052029, December 2012. doi:10.1088/1742-6596/400/5/052029.

[96] G. Armstrong, A. S. Greenberg, and J. R. Sites. Very low temperature thermal conductivity and optical properties of Stycast 1266 epoxy. Review of Scientific Instruments, 49(3):345-347, March 1978. doi:10.1063/1.1135404.

[97] J. R. Olson. Thermal conductivity of some common cryostat materials between 0.05 and 2 K. Cryogenics, 33(7):729-731, July 1993. doi:10.1016/0011-2275(93)90027-L.

[98] A. B. Zorin. The thermocoax cable as the microwave frequency filter for single-electron circuits. Review of Scientific Instruments, 66(8):4296-4300, August 1995. doi:10.1063/1.1145385.

[99] R. Stolle. Electromagnetic coupling of twisted pair cables. IEEE Journal on Selected Areas in Communications, 20(5):883-892, June 2002. doi:10.1109/JSAC.2002.1007371.

[100] K. Bladh, D. Gunnarsson, E. Hürfeld, S. Devi, C. Kristoffersson, B. Smålander, S. Pehrson, T. Claeson, P. Delsing, and M. Taslakov. Comparison of cryogenic filters for use in single electronics experiments. Review of Scientific Instruments, 74(3):1323-1327, March 2003. doi:10.1063/1.1540721.

[101] F. P. Milliken, J. R. Rozen, G. A. Keefe, and R. H. Koch. $50 \Omega$ characteristic impedance low-pass metal powder filters. Review of Scientific Instruments, 78(2):024701, February 2007. doi:10.1063/1.2431770.

[102] K. P. Hirvi, J. P. Kauppinen, A. N. Korotkov, M. A. Paalanen, and J. P. Pekola. Coulomb blockade thermometry. Czechoslovak Journal of Physics, 46(6):3345-3352, August 1996. doi:10.1007/BF02548151.

[103] N. Yurttagül, M. Sarsby, and A. Geresdi. Indium as a high-cooling-power nuclear refrigerant for quantum nanoelectronics. Physical Review Applied, 12(1):011005, July 2019. doi:10.1103/PhysRevApplied.12.011005.

[104] J. P. Pekola, J. J. Toppari, J. P. Kauppinen, K. M. Kinnunen, A. J. 
Manninen, and A. G. M. Jansen. Coulomb blockade-based nanothermometry in strong magnetic fields. Journal of Applied Physics, 83 (10):5582-5584, May 1998. doi:10.1063/1.367397.

[105] M. Watanabe, M. Morishita, and Y. Ootuka. Magnetoresistance of $\mathrm{RuO}_{2}$-based resistance thermometers below $0.3 \mathrm{~K}$. Cryogenics, 41(3): 143-148, March 2001. doi:10.1016/S0011-2275(01)00066-2.

[106] M. Meschke, J. P. Pekola, F. Gay, R. E. Rapp, and H. Godfrin. Electron thermalization in metallic islands probed by Coulomb blockade thermometry. Journal of Low Temperature Physics, 134(5):1119-1143, March 2004. doi:10.1023/B:JOLT.0000016733.75220.5d.

[107] K. L. Viisanen and J. P. Pekola. Anomalous electronic heat capacity of copper nanowires at sub-Kelvin temperatures. Physical Review B, 97(11): 115422, March 2018. doi:10.1103/PhysRevB.97.115422.

[108] W. A. Little. The transport of heat between dissimilar solids at low temperatures. Canadian Journal of Physics, 37(3):334-349, March 1959. doi:10.1139/p59-037.

[109] R. E. Peterson and A. C. Anderson. Transport of heat between electrons and phonons in copper below 0.2 K. Physica Status Solidi (B), 56(1): 243-247, March 1973. doi:10.1002/pssb.2220560123.

[110] S. Autti, K. Grigoras, A. M. Guénault, D. Gunnarsson, R. P. Haley, A. T. Jones, Yu. Pashkin, J. R. Prance, M. Prunnila, L. Roschier, and D. E. Zmeev. Microkelvin-ready Coulomb blockade thermometry with on-chip cooling. To be published, 2019.

[111] A. Bjorneklett, L. Halbo, and H. Kristiansen. Thermal conductivity of epoxy adhesives filled with silver particles. International Journal of Adhesion and Adhesives, 12(2):99-104, April 1992. doi:10.1016/0143-7496(92)90030-Y.

[112] R. B. Stephens. The thermal properties of sample addenda used in T1 K specific heat measurements: 1 - Specific heat and thermal conductivity of General Electric 7031 varnish. Cryogenics, 15(7):420-422, July 1975. doi:10.1016/0011-2275(75)90012-0.

[113] G. Frossati. Obtaining ultralow temperatures by dilution of 3He into $4 \mathrm{He}$. Journal de Physique Colloques, 39(C6):1578-1589, August 1978. doi:10.1051/jphyscol:19786604. 
[114] R. Blaauwgeers, M. Blazkova, M. Človečko, V. B. Eltsov, R. de Graaf, J. Hosio, M. Krusius, D. Schmoranzer, W. Schoepe, L. Skrbek, P. Skyba, R. E. Solntsev, and D. E. Zmeev. Quartz tuning fork: thermometer, pressure- and viscometer for helium liquids. Journal of Low Temperature Physics, 146(5):537-562, March 2007. doi:10.1007/s10909-006-9279-4.

[115] D. I. Bradley, M. Človečko, S. N. Fisher, D. Garg, A. M. Guénault, E. Guise, R. P. Haley, G. R. Pickett, M. Poole, and V. Tsepelin. Thermometry in normal liquid $3 \mathrm{He}$ using a quartz tuning fork viscometer. Journal of Low Temperature Physics, 171(5):750-756, June 2013. doi:10.1007/s10909-012-0804-3.

[116] D. I. Bradley and R. Oswald. Viscosity of the $3 \mathrm{He}-4 \mathrm{He}$ dilute phase in the mixing chamber of a dilution refrigerator. Journal of Low Temperature Physics, 80(1):89-97, July 1990. doi:10.1007/BF00683116.

[117] J. C. H. Zeegers, A. Th. A. M. de Waele, and H. M. Gijsman. Viscosity of saturated 3He-4He mixture below $200 \mathrm{mK}$. Journal of Low Temperature Physics, 84(1):37-47, July 1991. doi:10.1007/BF00681616.

[118] S. T. Boldarev, R. B. Gusev, S. I. Danilin, and A. Ya. Parshin. Use of a quartz resonator of the tuning fork type as a thermometer in a dilution refrigerator. Instruments and Experimental Techniques, 54(5):740-747, October 2011. doi:10.1134/S0020441211050101.

[119] E. Pentti, J. Rysti, A. Salmela, A. Sebedash, and J. Tuoriniemi. Studies on helium liquids by vibrating wires and quartz tuning forks. Journal of Low Temperature Physics, 165(3):132-165, September 2011. doi:10.1007/s10909-011-0394-5.

[120] W. C. Black, W. R. Roach, and J. C. Wheatley. Speer carbon resistors as thermometers for use below 1K. Review of Scientific Instruments, 35(5): 587-591, May 1964. doi:10.1063/1.1718880.

[121] J. Sanchez, A. Benoit, and J. Flouquet. Speer carbon resistance thermometer magnetoresistance effect. Review of Scientific Instruments, 48 (8):1090-1091, August 1977. doi:10.1063/1.1135190.

[122] K. Yamazaki, K. Kato, K. Muramatsu, A. Haga, K. Kobayashi, K. Kamata, K. Fujiwara, and T. Yamaguchi. Incremental permeability of mu-metal in low magnetic fields for the design of multilayer-type magnetically shielded rooms. IEEE Transactions on Magnetics, 41(10): 4087-4089, October 2005. doi:10.1109/TMAG.2005.855196. 
[123] R. Kalra, A. Laucht, J. P. Dehollain, D. Bar, S. Freer, S. Simmons, J. T. Muhonen, and A. Morello. Vibration-induced electrical noise in a cryogen-free dilution refrigerator: characterization, mitigation, and impact on qubit coherence. Review of Scientific Instruments, 87(7):073905, June 2016. doi:10.1063/1.4959153.

[124] M. de Wit, G. Welker, K. Heeck, F. M. Buters, H. J. Eerkens, G. Koning, H. van der Meer, D. Bouwmeester, and T. H. Oosterkamp. Vibration isolation with high thermal conductance for a cryogen-free dilution refrigerator. Review of Scientific Instruments, 90(1):015112, January 2019. doi:10.1063/1.5066618.

[125] E. Leobandung, L. Guo, Y. Wang, and S. Y. Chou. Observation of quantum effects and Coulomb blockade in silicon quantum-dot transistors at temperatures over 100 K. Applied Physics Letters, 67(7):938-940, August 1995. doi:10.1063/1.114701.

[126] Yu. A. Pashkin, Y. Nakamura, and J. S. Tsai. Room-temperature Al single-electron transistor made by electron-beam lithography. Applied Physics Letters, 76(16):2256-2258, 2000. doi:10.1063/1.126313.

[127] M. Sarsby. Nanoelectronic and nanomechanical devices for low temperature applications. PhD thesis, Lancaster University, January 2017. URL http://www.research.lancs.ac.uk/portal/en/publications/ nanoelectronic-and-nanomechanical-devices-for-low-temperatureapplications (0c112367-e401-4552-9247-cdaa2a2e3dc7) .html.

[128] K. H. Ang, G. Chong, and Y. Li. PID control system analysis, design, and technology. IEEE Transactions on Control Systems Technology, 13(4): 559-576, July 2005. doi:10.1109/TCST.2005.847331.

[129] M. J. Biercuk, D. J. Monsma, C. M. Marcus, J. S. Becker, and R. G. Gordon. Low-temperature atomic-layer-deposition lift-off method for microelectronic and nanoelectronic applications. Applied Physics Letters, 83(12):2405-2407, September 2003. doi:10.1063/1.1612904.

[130] K. Z. Rajab, M. Naftaly, E. H. Linfield, J. C. Nino, D. Arenas, D. Tanner, R. Mittra, and M. Lanagan. Broadband dielectric characterization of aluminum oxide (Al2O3). Journal of Microelectronics and Electronic Packaging, 5(1):2-7, January 2008. doi:10.4071/1551-4897-5.1.1.

[131] L.-Y. Chen and G. W. Hunter. Temperature dependent dielectric 
properties of polycrystalline 96\% Al2O3. MRS Proceedings, 833:249-254, December 2004. doi:10.1557/PROC-833-G7.6.

[132] J. Acharya, J. Wilt, B. Liu, and J. Wu. Probing the dielectric properties of ultrathin $\mathrm{Al} / \mathrm{Al} 2 \mathrm{O} 3 / \mathrm{Al}$ trilayers fabricated using in situ sputtering and atomic layer deposition. ACS Applied Materials \& Interfaces, 10(3): 3112-3120, January 2018. doi:10.1021/acsami.7b16506.

[133] D. I. Bradley, M. R. Follows, I. E. Miller, R. Oswald, and M. Ward. Simple design for dilution refrigerator with base temperature of $2.3 \mathrm{mK}$. Cryogenics, 34(6):549-550, June 1994. doi:10.1016/0011-2275(94)90217-8.

[134] M. Palma, C. P. Scheller, D. Maradan, A. V. Feshchenko, M. Meschke, and D. M. Zumbühl. On-and-off chip cooling of a Coulomb blockade thermometer down to $2.8 \mathrm{mK}$. Applied Physics Letters, 111(25):253105, December 2017. doi:10.1063/1.5002565.

[135] I. A. Gachechiladze, D. V. Pavlov, and A. V. Pantsulaya. Optimization of nuclear demagnetization process. Cryogenics, 26(4):242-247, April 1986. doi:10.1016/0011-2275(86)90206-7.

[136] P. G. Strehlow. Optimized thermodynamic process of nuclear cooling. AIP Conference Proceedings, 850(1):1575-1576, September 2006. doi:10.1063/1.2355307.

[137] J. Vonka. Demagnetisation of solid 3 He and supercritical superflow. PhD thesis, Lancaster University, February 2018. doi:10.17635/lancaster/thesis/231.

[138] D. Jiles. Introduction to magnetism and magnetic materials. CRC press, 2015. ISBN 978-0-412-79860-3. doi:10.1201/b18948.

[139] M. Schwark, F. Pobell, W. P. Halperin, Ch. Buchal, J. Hanssen, M. Kubota, and R. M. Mueller. Ortho-para conversion of hydrogen in copper as origin of time-dependent heat leaks. Journal of Low Temperature Physics, 53(5):685-694, December 1983. doi:10.1007/BF00684000.

[140] V. Pecharsky and P. Zavalij. Fundamentals of powder diffraction and structural characterization of materials. Springer Science \& Business Media, 2008. ISBN 0-387-24147-7. doi:10.1007/b106242.

[141] T. Hara, T. Yamasaki, and K. Kinoshita. Grain sizes in electroplated thin copper interconnection layers. Journal of the Electrochemical Society, 153 (12):G1059-G1063, October 2006. doi:10.1149/1.2358933. 
[142] H. E. Swanson and E. Tatge. Standard X-ray diffraction powder patterns, volume 1 Data for 54 inorganic substances of National Bureau of Standards circular 539. National Bureau of Standards, Washington, D.C., June 1953. URL https://ntrl.ntis.gov/NTRL/dashboard/searchResults/ titleDetail/PB178902.xhtml.

[143] S. Hüfner. Photoelectron spectroscopy: principles and applications. Springer Science \& Business Media, 2013. ISBN 978-3-642-07520-9. doi:10.1007/978-3-662-09280-4.

[144] F. Stevie. Secondary ion mass spectrometry: applications for depth profiling and surface characterization. Momentum Press, 2015. ISBN 978-1-60650-588-5. URL http:

//www .momentumpress . net/books/secondary-ion-mass-spectrometryapplications-depth-profiling-and-surface-characterization.

[145] M. Palma, D. Maradan, L. Casparis, T.-M. Liu, F. N. M. Froning, and D. M. Zumbühl. Magnetic cooling for microkelvin nanoelectronics on a cryofree platform. Review of Scientific Instruments, 88(4):043902, April 2017. doi:10.1063/1.4979929.

[146] M. Palma. Magnetic cooling and on-chip thermometry for nanoelectronics below $10 \mathrm{mK}$. PhD thesis, University of Basel, November 2017. doi:10.5451/unibas-006754527.

[147] Y. B. Kalyoncu. Hydrogen plasma etched graphene nanoribbons. PhD thesis, University of Basel, January 2019. doi:10.5451/unibas-007056161.

[148] A. Vlasov, J. Guillemette, G. Gervais, and T. Szkopek. Magnetic refrigeration with paramagnetic semiconductors at cryogenic temperatures. Applied Physics Letters, 111(14):142102, February 2019. doi:10.1063/1.4994536.

[149] R. W. Cochrane, R. Harris, J. O. Ström-Olson, and M. J. Zuckermann. Structural manifestations in amorphous alloys: resistance minima. Physical Review Letters, 35(10):676-679, September 1975. doi:10.1103/PhysRevLett.35.676.

[150] J. Kondo. Resistance minimum in dilute magnetic alloys. Progress of Theoretical Physics, 32(1):37-49, July 1964. doi:10.1143/PTP.32.37.

[151] J. R. A. Dann, P. C. Verpoort, J. Ferreira de Oliveira, S. E. Rowley, A. Datta, S. Kar-Narayan, C. J. B. Ford, G. J. Conduit, and V. Narayan. 
Au-Ge alloys for wide-range low-temperature on-chip thermometry.

Physical Review Applied, 12(3):034024, September 2019.

doi:10.1103/PhysRevApplied.12.034024. 